Luíze Mello D’Urso Vianna

\title{
Transformações de Cremona como Isometrias
} Hiperbólicas

Dissertação de Mestrado

Dissertação apresentada como requisito parcial para obtenção do grau de Mestre pelo Programa de Pós-graduação em Matemática, do Departamento de Matemática da PUC-Rio.

Orientador : Prof. Nicolau Corcao Saldanha

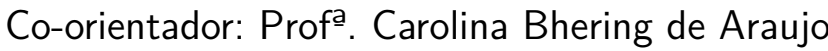




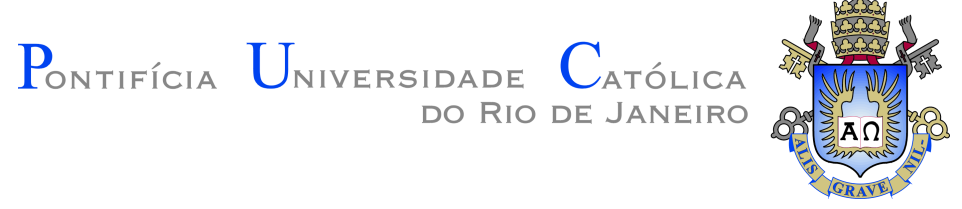

Luíze Mello D'Urso Vianna

\section{Transformações de Cremona como Isometrias Hiperbólicas}

Dissertação apresentada como requisito parcial para obtenção do grau de Mestre pelo Programa de Pós-graduação em Matemática da PUC-Rio. Aprovada pela Comissão Examinadora abaixo:

Prof. Nicolau Corcao Saldanha

Orientador

Departamento de Matemática - PUC-Rio

Profa. Carolina Bhering de Araujo

IMPA

Profa. Jacqueline Fabiola Rojas Arancibia

UFPB

Dr. Lucas de Souza das Dores

IMPA

Profa. Viviana Ferrer Cuadrado

UFF

Rio de Janeiro, 26 de Fevereiro de 2021 
Todos os direitos reservados. A reprodução, total ou parcial do trabalho, é proibida sem a autorização da universidade, do autor e do orientador.

\section{Luíze Mello D'Urso Vianna}

Bacharel em matemática pela PUC-Rio 2015-2018, Mestre na área de Geometria Algébrica pela mesma instituição em 2021 e atualmente estudante de Doutorado no IMPA. Medalha de ouro na OBMEP de 2008 a 2014; OBM 2011 e 2018; OMERJ 2011, 2013, 2017; CIIM 2017 (Competencia Iberoamericana Interuniversitaria de Matemáticas) em Quito, Equador. Além disso participou da IMC 2016, 2017 (International Mathematics Competition for University Students) em Blagoevgrad, Bulgária conquistando medalhas de bronze. Também foi vice-líder da equipe da EGMO (European Girls Mathematics Olympiad) 2018 em Florença, Itália e 2019 em Kiev, Ucrânia e líder 2021 remotamente. Vencedora do prêmio Olímpicas 2018. Criou o projeto Matemática para Garotas em 2018 ( @mathforgirls ), onde leciona semanalmente, com o objetivo de ampliar a participação de meninas nas olimpíadas de matemática. Participou do programa Simons Visitor do ICTP (International Centre for Theoretical Physics) em 2019 Tieste, Itália.

Ficha Catalográfica

D'Urso, Luíze

Transformações de Cremona como Isometrias Hiperbólicas / Luíze Mello D'Urso Vianna; orientador: Nicolau Corcao Saldanha; co-orientador: Carolina Bhering de Araujo. - 2021.

85 f: il. color. ; $30 \mathrm{~cm}$

Dissertação (mestrado) - Pontifícia Universidade Católica do Rio de Janeiro, Departamento de Matemática, 2021.

Inclui bibliografia

1. Matemática - Teses. 2. Transformações de Cremona. 3. Isometrias Hiperbólicas. 4. Grupo de Cremona. 5. Geometria Birracional. I. Saldanha, Nicolau. II. Araujo, Carolina. III. Pontifícia Universidade Católica do Rio de Janeiro. Departamento de Matemática. IV. Título. 
À minha família, pelo apoio.

Aos meus amigos da Salinha, pela inspiração.

Ao meu companheiro, em especial, pelo incentivo. 


\section{Agradecimentos}

Agradeço ao meu orientador Nicolau Saldanha, pelas muitas oportunidades que tive desde a graduação, que me permitiram chegar até aqui. A ele também devo um olhar único sobre a Álgebra.

Agradeço à minha orientadora Carolina Araujo, que me acolheu e me sugeriu a linha de pesquisa do presente trabalho. Agradeço por todo o apoio até o final e por sempre destacar a Geometria na minha vida.

Agradeço à bolsa Arquimedes, cujo incentivo tornou possível este trabalho.

O presente trabalho foi realizado com apoio da Coordenação de Aperfeiçoamento de Pessoal de Nível Superior - Brasil (CAPES) - Código de Financiamento 001. 


\section{Resumo}

D’Urso, Luíze; Saldanha, Nicolau; Araujo, Carolina. Transformações de Cremona como Isometrias Hiperbólicas. Rio de Janeiro, 2021. 85p. Dissertação de Mestrado - Departamento de Matemática, Pontifícia Universidade Católica do Rio de Janeiro.

O Grupo de Cremona é o grupo das Transformações birracionais do plano projetivo e tem um papel muito importante em Geometria Birracional. Pelo Teorema de Nöether-Castelnuovo (final do século XIX), o Grupo de Cremona é gerado pelos automorfismos do plano projetivo e pela Transformação Quadrática Padrão $\Phi$ dada por $(x: y: z) \mapsto(y z: x z: x y)$. Apesar de compreendermos bem o grupo de automorfismos do Plano Projetivo e a involução $\Phi$, o estudo do Grupo de Cremona é bastante desafiador, e sua estrutura ainda não é totalmente conhecida.

Somente em 2013, Cantat e Lamy provaram que o Grupo de Cremona não é simples no caso de um corpo algebricamente fechado. Em 2016, Anne Lonjou provou o mesmo para qualquer corpo. Ambas as provas se baseiam em uma ação por isometrias do Grupo de Cremona em um espaço hiperbólico de dimensão infinita. Nosso objetivo será entender essa ação e como ela pode ser usada no estudo do Grupo de Cremona.

\section{Palavras-chave}

Transformações de Cremona; Isometrias Hiperbólicas; Grupo de Cremona; Geometria Birracional. 


\section{Abstract}

D'Urso, Luíze; Saldanha, Nicolau (Advisor); Araujo, Carolina (CoAdvisor). Cremona Transformations as Hiperbolic Isometries. Rio de Janeiro, 2021. 85p. Dissertação de Mestrado - Departamento de Matemática, Pontifícia Universidade Católica do Rio de Janeiro.

The Cremona Group is the group of Birrational Transformations of the projective plane and has a very important role in Birrational Geometry. By the Nöether-Castelnuovo Theorem (late 19th century), the Cremona Group is generated by the automorphisms of the projective plane and by the Standard Quadratic Transformation $\Phi$ given by $(x: y: z) \mapsto(y z: x z: x y)$. Although we understand well the group of automorphisms of the projective plane and the involution $\Phi$, the study of the Cremona Group is quite challenging, and its structure is not yet fully known.

Only in 2013, Cantat and Lamy proved that the Cremona Group is not simple in the case of an algebraically closed field. In 2016, Anne Lonjou proved the same for any field. Both proofs are based on an action by isometries of the Cremona Group in a hyperbolic space of infinite dimension. Our goal will be to understand this action and how it can be used in the study of the Cremona Group.

\section{Keywords}

Cremona Transformations; Hiperbolic Isometries; Cremona Group; Birracional Geometry. 


\section{Sumário}

$\begin{array}{lll}1 & \text { Introdução } & 10\end{array}$

2 Variedades Algébricas $\quad 15$

2.1 O espaço afim e conjuntos algébricos afins 15

$\begin{array}{lll}2.2 & \text { O espaço projetivo e conjuntos algébricos projetivos } & 17\end{array}$

$\begin{array}{lll}2.3 & \text { A topologia de Zariski } & 20\end{array}$

$\begin{array}{lll}2.4 & \text { O produto cartesiano de variedades algébricas } & 22\end{array}$

3 Morfismos e aplicações racionais $\quad 26$

3.1 Morfismos e funções regulares $\quad 26$

3.2 Aplicações racionais 31

3.3 Funções racionais e dimensão 35

4 Blowup de superfícies $\quad 38$

$\begin{array}{lll}4.1 & \text { O blowup de } \mathbb{P}^{2} \text { em um ponto } & 39\end{array}$

4.2 O blowup de $\mathbb{P}^{2}$ em mais pontos 41

4.3 Resolução de indeterminação $\quad 42$

5 Divisores $\quad 47$

$\begin{array}{lll}5.1 & \text { O Grupo de Picard } & 47\end{array}$

5.2 O pullback de divisores $\quad 51$

$\begin{array}{ll}5.3 \text { A forma de interseção } & 57\end{array}$

6 Interações com Geometria Hiperbólica 62

6.1 Motivação 62

6.2 O modelo do hiperbolóide para $\mathbb{H}^{n} \quad 65$

$\begin{array}{lll}6.3 & \text { Isometrias de } \mathbb{H}^{n} & 67\end{array}$

$\begin{array}{lll}7 & \text { A ação do Grupo de Cremona } & \mathbf{7 0}\end{array}$

$\begin{array}{lll}7.1 & \text { O Espaço de Picard-Manin } & 70\end{array}$

$\begin{array}{lll}7.2 & \text { O modelo do hiperbolóide em dimensão infinita } & 73\end{array}$

$\begin{array}{ll}7.3 \text { A ação } & 74\end{array}$

8 Não simplicidade do Grupo de Cremona $\quad 78$

$\begin{array}{lll}8.1 \text { A propriedade WPD } & 79\end{array}$

$\begin{array}{lll}8.2 \text { Os elementos } h_{n} & 80\end{array}$

9 Referências bibliográficas $\quad 85$ 
The beauty of mathematics only shows itself to more patient followers.

Maryam Mirzakhani. 


\section{1 \\ Introdução}

Dado um corpo $k$, considere o plano projetivo

$$
\mathbb{P}_{k}^{2}=\frac{k^{3} \backslash\{(0,0,0)\}}{\sim}
$$

onde a relação $\sim$ é dada pelo seguinte: $\left(p_{0}, p_{1}, p_{2}\right) \sim\left(q_{0}, q_{1}, q_{2}\right)$ se existe um escalar não nulo $\lambda \in k \backslash\{0\}$ tal que $p_{i}=\lambda q_{i}$ para todo $i=0,1,2$. Uma interpretação geométrica é que $\mathbb{P}_{k}^{2}$ tem como elementos as retas em $k^{3}$ passando pela origem. Usamos a notação $\left(p_{0}: p_{1}: p_{2}\right)$ para descrever a classe do ponto $\left(p_{0}, p_{1}, p_{2}\right) \in k^{3} \backslash\{(0,0,0)\}$ em $\mathbb{P}_{k}^{2}$.

Dados três polinômios homogêneos $F, G, H$ em três variáveis com coeficientes em $k$, todos de mesmo grau $d \geq 1$, isto é, $F, G, H \in k[x, y, z]_{d}$, e sem fatores comuns, podemos definir uma aplicação racional $\varphi: \mathbb{P}_{k}^{2} \rightarrow \mathbb{P}_{k}^{2}$, dada por $(x: y: z) \mapsto(F(x, y, z): G(x, y, z): H(x, y, z))$.

Note que alguns pontos em $\mathbb{P}_{k}^{2}$ podem não ter uma imagem bem definida. Isso ocorre quando $F\left(p_{0}, p_{1}, p_{2}\right)=G\left(p_{0}, p_{1}, p_{2}\right)=H\left(p_{0}, p_{1}, p_{2}\right)=0$. Nesse caso, dizemos que $\left(p_{0}: p_{1}: p_{2}\right)$ é um ponto de indeterminação de $\varphi$. Caso não existam pontos de indeterminação, como por exemplo na aplicação $(x: y: z) \mapsto$ $\left(x^{2}: y^{2}: z^{2}\right)$, então esta aplicação racional é dita um morfismo, e é denotada por $\varphi: \mathbb{P}_{k}^{2} \rightarrow \mathbb{P}_{k}^{2}$, com a seta inteira.

Uma aplicação racional $\varphi: \mathbb{P}_{k}^{2} \rightarrow \mathbb{P}_{k}^{2}$ é dita birracional, ou Transformação de Cremona, se existe uma aplicação racional $\psi: \mathbb{P}_{k}^{2} \rightarrow \mathbb{P}_{k}^{2}$ tal que $\psi \circ \varphi$ e $\varphi \circ \psi$ coincidem com a identidade em um aberto denso de $\mathbb{P}_{k}^{2}$ (ver Capítulo 2.3 para o estudo da topologia de Zariski). Quando nem $\varphi$ nem $\psi$ possuem pontos de indeterminação, dizemos que são automorfismos de $\mathbb{P}_{k}^{2}$. A noção de aplicação racional e morfismo se estende para outras variedades projetivas além de $\mathbb{P}_{k}^{2}$ de forma local.

Um exemplo de Transformação de Cremona é a Transformação Quadrática Padrão, sempre denotada por $\Phi: \mathbb{P}_{k}^{2} \rightarrow \mathbb{P}_{k}^{2}$, dada por

$$
(x: y: z) \mapsto(y z: x z: x y)
$$

Sua aplicação inversa é ela mesma, isto é, $\Phi \circ \Phi \operatorname{satisfaz}(x: y: z) \mapsto(x: y: z)$ no aberto $x y z \neq 0$.

A Transformação Quadrática Padrão possui três pontos de indeterminação, que são exatamente os pontos coordenados $P_{0}=(1: 0: 0), P_{1}=(0: 1: 0), P_{2}=$ (0:0:1). Além disso, ela contrai as retas coordenadas $L_{12}, L_{02}, L_{01}$, dadas res- 
pectivamente pelas equações $x=0, y=0, z=0$, nos pontos coordenados.

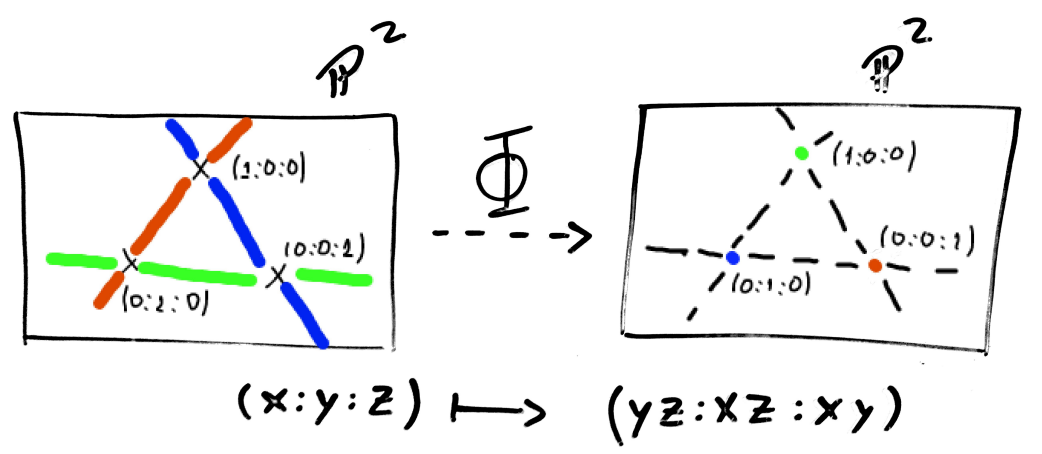

O grupo das Transformações de Cremona é conhecido como o Grupo de Cremona de $\mathbb{P}_{k}^{2}$, ou $\operatorname{Cr}\left(\mathbb{P}_{k}^{2}\right)$, e será nosso principal objeto de estudo. Também é possível tratar o Grupo de Cremona de um ponto de vista puramente algébrico, como o grupo de $k$-automorfismos do corpo de frações $k(x, y)$ de $k$ em duas variáveis. Isso está justificado na Capítulo 3.3.

O Teorema de Nöether-Castelnuovo (em, por exemplo, (CASTELNUOVO, 1901)) estabelece que $\mathrm{Cr}\left(\mathbb{P}_{k}^{2}\right)$ é o grupo gerado pelos automorfismos de $\mathbb{P}_{k}^{2}$ e a Transformação Quadrática Padrão $\Phi$, isto é, $\operatorname{Cr}\left(\mathbb{P}_{k}^{2}\right)=\left\langle\operatorname{Aut}\left(\mathbb{P}_{k}^{2}\right), \Phi\right\rangle$. Apesar de $\operatorname{Aut}\left(\mathbb{P}_{k}^{2}\right) \cong \operatorname{PGL}(3, k)$ ser um grupo bem conhecido, acrescentar a única Transformação de Cremona $\Phi$ como geradora torna o Grupo de Cremona de $\mathbb{P}_{k}^{2}$ extremamente complicado. Algumas questões fundamentais, como por exemplo decidir se $\operatorname{Cr}\left(\mathbb{P}_{k}^{2}\right)$ é simples, foram respondidas apenas muito recentemente.

Em (CANTAT; LAMY, 2013), Serge Cantat e Stéphane Lamy mostraram que o Grupo de Cremona de $\mathbb{P}_{k}^{2}$ não é simples quando $k$ é algebricamente fechado. O artigo explora uma ação de grupo de $\operatorname{Cr}\left(\mathbb{P}_{k}^{2}\right)$ em um espaço hiperbólico de dimensão infinita por isometrias, e usa ferramentas de Geometria Hiperbólica para obter o resultado. Em 2016, em (LONJOU, 2016), Anne Lonjou prova o mesmo resultado para qualquer corpo, usando a mesma ação.

O objetivo deste estudo é compreender como Transformações de Cremona podem ser vistas como isometrias hiperbólicas, ou seja, entender a ação de $\operatorname{Cr}\left(\mathbb{P}_{k}^{2}\right)$ por isometrias mencionada acima e quais conclusões podemos obter dela. O que vem a seguir é um pequeno vislumbre do que veremos ao longo deste trabalho. 
Para entender melhor as Transformações de Cremona, vamos considerar blowups de $\mathbb{P}_{k}^{2}$. Podemos entender o blowup de uma superfície $S$ em um ponto $P \in S$ como uma modificação de $S$ onde substituímos $P$ por uma curva isomorfa a $\mathbb{P}_{k}^{1}$. Cada ponto desta curva corresponde a uma direção tangente a $S$ em $P$. Mais precisamente, o blowup de $S$ em $P$ é um morfismo $\pi: S_{P} \rightarrow S$, de forma que $\mathcal{E}=\pi^{-1}(P) \cong \mathbb{P}_{k}^{1}$ e $\pi$ induz um isomorfismo $S_{P} \backslash \mathcal{E} \stackrel{\sim}{\rightarrow} S \backslash\{P\}$.

A importância do blowup no nosso estudo se dá por podermos resolver as indeterminações de qualquer Transformação de Cremona através de uma sequência de blowups. Mais precisamente, para toda Transformação de Cremona $\varphi: \mathbb{P}_{k}^{2} \rightarrow \mathbb{P}_{k}^{2}$, existe uma superfície não-singular $S$ e morfismos $\pi: S \rightarrow \mathbb{P}^{2}$ e $\widetilde{\varphi}: S \rightarrow \mathbb{P}^{2}$, ambos composições de finitos blowups, de modo que $\widetilde{\varphi}=\varphi \circ \pi$. Em outras palavras, a aplicação $\varphi \circ \pi$ não possui pontos de indeterminação.

Podemos por exemplo considerar novamente a Transformação Quadrática Padrão $\Phi: \mathbb{P}_{k}^{2} \rightarrow \mathbb{P}_{k}^{2}$. Ao tomarmos o blowup consecutivo de $\mathbb{P}_{k}^{2}$ nos três pontos de indeterminação de $\Phi$, conseguiremos resolver suas indeterminações, obtendo o morfismo $\widetilde{\Phi}: S \rightarrow \mathbb{P}_{k}^{2}$.

Mais ainda, veremos que $\Phi$ se levanta a um automorfismo $\widetilde{\widetilde{\Phi}}: S \rightarrow S$ do blowup final de $\mathbb{P}_{k}^{2}$ nos três pontos coordenados, ou seja, $\widetilde{\widetilde{\Phi}}=\pi^{-1} \circ \Phi \circ \pi$ é um automorfismo de $S$.

Estamos especialmente interessados na ação do automorfismo $\widetilde{\widetilde{\Phi}}$ nas curvas em $S$. Por exemplo, $\widetilde{\widetilde{\Phi}}$ leva isomorficamente a curva $\pi^{-1}\left(P_{0}\right)$ no fecho topológico de $\pi^{-1}\left(L_{12} \backslash\left\{P_{1}, P_{2}\right\}\right)$.

Ao estudar as curvas de $S$, somos levados a considerar o Grupo de Picard de $S, \operatorname{Pic}(S) \cong \mathbb{Z}^{4}$ (Capítulo 5.1), e sua extensão linear $\operatorname{Pic}(S) \otimes_{\mathbb{Z}} \mathbb{R} \cong \mathbb{R}^{4}$. Ocorre que $\widetilde{\widetilde{\Phi}}$ induz de forma natural um automorfismo linear de $\mathbb{R}^{4}$.

No Grupo de Picard de qualquer superfície, temos uma forma bilinear simétrica que é preservada por isomorfismos. Para a superfície $S$ definida acima, por exemplo, essa forma é de assinatura (1,3), isto é, com uma escolha adequada de base para $\operatorname{Pic}(S)$, temos

$$
\left(a_{0}, a_{1}, a_{2}, a_{3}\right) \cdot\left(b_{0}, b_{1}, b_{2}, b_{3}\right)=a_{0} b_{0}-a_{1} b_{1}-a_{2} b_{2}-a_{3} b_{3} .
$$

Esta forma nos remete ao Espaço de Minkowski, isto é, $\left(\mathbb{R}^{4}, \cdot\right)$, onde se define o modelo do hiperbolóide para o Espaço Hiperbólico $\mathbb{H}^{3}$.

Neste modelo, $\mathbb{H}^{3}=\left\{v=\left(v_{0}, v_{1}, v_{2}, v_{3}\right) \in \mathbb{R}^{4} \mid v \cdot v=1, v_{0}>0\right\}$ é uma folha de hiperbolóide em $\mathbb{R}^{4}$, e possui uma distância dada por $\mathrm{d}(u, v)=$ $\cosh ^{-1}(u \cdot v)$. Veremos então que a transformação linear $\mathbb{R}^{4} \rightarrow \mathbb{R}^{4}$ induzida pelo automorfismo $\widetilde{\widetilde{\Phi}}: S \rightarrow S$ preserva a folha de hiperbolóide e a distância entre quaisquer dois de seus pontos. Portanto, $\widetilde{\widetilde{\Phi}}$ induz uma isometria em $\mathbb{H}^{3}$. 


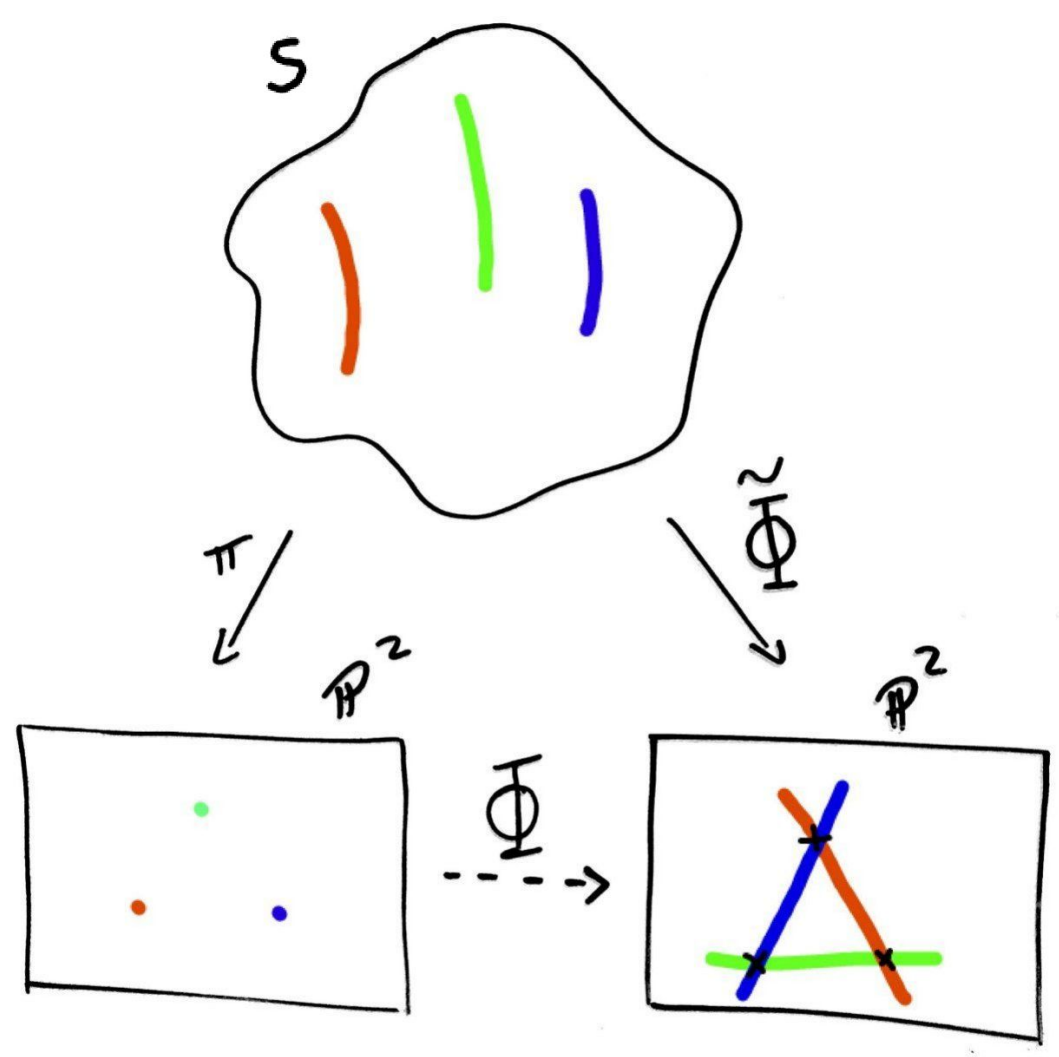

Infelizmente, nem toda Transformação de Cremona pode ser conjugada a um automorfismo como no caso da Transformação Quadrática Padrão. Ainda assim, neste estudo vamos construir uma ação de $\operatorname{Cr}\left(\mathbb{P}_{k}^{2}\right)$ por isometrias em um espaço hiperbólico de dimensao infinita.

Em seguida, vamos investigar como propriedades álgebro-geométricas de $\varphi \in \operatorname{Cr}\left(\mathbb{P}_{k}^{2}\right)$ podem ser detectadas a partir da isometria associada. Por exemplo, sabemos classificar isometrias de um espaço hiperbólico como elíptica, parabólica ou hiperbólica, consequentemente podemos classificar também as Transformações de Cremona. Em (LONJOU, 2016), é dada a construção de uma Transformação de Cremona hiperbólica $h$ que possui alguma potência $h^{n}$ que gera um subgrupo normal próprio de $\operatorname{Cr}\left(\mathbb{P}_{k}^{2}\right)$, provando que este grupo não é simples.

A seguir, descrevemos como esta dissertação está organizada. 


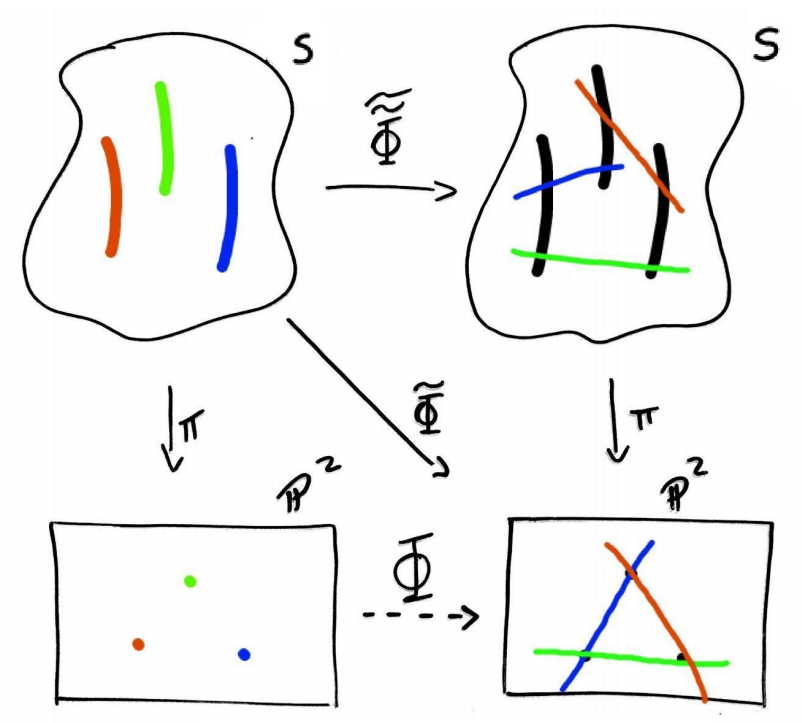

O Capítulo 2 introduz objetos básicos estudados em Geometria Algébrica. Inclui definições de variedades afins, variedades projetivas e quasi-projetivas, descreve a topologia de Zariski.

O Capítulo 3 dá definições mais precisas de morfismos e aplicações racionais.

(Os Capítulos 2 e 3 são introdutórios, e podem ser ignorados por um leitor que já conheça os conceitos básicos de Geometria Algébrica clássica.)

O Capítulo 4 aborda blowups de superfícies não-singulares, dá uma descrição do blowup de $\mathbb{P}_{k}^{2}$ em um ponto em coordenadas e os principais Teoremas de resolução de indeterminação de aplicações racionais em superfícies.

O Capítulo 5 apresenta divisores e o Grupo de Picard de uma superfície não-singular, introduz o pullback de um divisor, descreve a forma de interseção em Grupos de Picard.

O Capítulo 6 discute o modelo do hiperbolóide do espaço hiperbólico de dimensão finita e sua interação com subgrupos finitos do Grupo de Cremona sobre os Complexos.

O Capítulo 7 descreve o espaço de Picard-Manin $\mathbb{H}^{\infty}$, um espaço hiperbólico de dimensão infinita e a ação do Grupo de Cremona por isometrias em $\mathbb{H}^{\infty}$.

O Capítulo 8 tem um pequeno resumo de (LONJOU, 2016), onde é demonstrada a não simplicidade do Grupo de Cremona para qualquer corpo $k$, uma poderosa consequência da ação descrita no Capítulo anterior. 


\section{2}

\section{Variedades Algébricas}

Usaremos a notação $k\left[x_{0}, x_{1}, \ldots, x_{n}\right]$ para o conjunto dos polinômios com coeficientes em $k$ em $n+1$ variáveis e $k\left[x_{0}, x_{1}, \ldots, x_{n}\right]^{h}$ para o seu subconjunto de polinômios homogêneos, cujos elementos serão sempre denotados por uma letra maiúscula. Para referências, considere (SHAFAREVICH, 1994) e (HARTSHORNE, 1977).

\section{1}

\section{O espaço afim e conjuntos algébricos afins}

O espaço afim de dimensão $n$ sobre um corpo $k$, como conjunto, é simplesmente $\mathbb{A}_{k}^{n}=k^{n}$. Vale notar que $\mathbb{A}_{k}^{n}$ não está munido da estrutura de espaço vetorial de $k^{n}$, mas futuramente será munido de uma estrutura de espaço topológico, com a topologia de Zariski. Nessa topologia, os fechados serão os conjuntos algébricos afins, que serão definidos a seguir.

Considere $f \in k\left[x_{1}, x_{2}, \ldots, x_{n}\right]$ um polinômio em $n$ variáveis com coeficientes em $k$. Vamos definir o conjunto de zeros de $f$ em $\mathbb{A}_{k}^{n}$ como

$$
\mathcal{Z}(f)=\left\{P \in \mathbb{A}_{k}^{n} \mid f(P)=0\right\},
$$

onde $f(P)=f\left(p_{1}, p_{2}, \ldots, p_{n}\right)$ para todo ponto $P=\left(p_{1}, p_{2}, \ldots, p_{n}\right) \in \mathbb{A}_{k}^{n}$.

Mais geralmente, podemos definir o conjunto de zeros de um conjunto $S \subset k\left[x_{1}, x_{2}, \ldots, x_{n}\right]$ de polinômios em $\mathbb{A}_{k}^{n}$ como

$$
\mathcal{Z}(S)=\left\{P \in \mathbb{A}_{k}^{n} \mid f(P)=0 \forall f \in S\right\}
$$

Um conjunto como este é chamado de conjunto algébrico afim, ou conjunto algébrico de $\mathbb{A}_{k}^{n}$.

Exemplo 1. Tanto $\mathbb{A}_{k}^{n}$ quanto $\varnothing$ são conjuntos algébricos, sendo respectivamente o conjunto de zeros dos polinômios $f=0$ e $g=1$.

Exemplo 2. Um ponto $P=\left(p_{1}, p_{2}, \ldots, p_{n}\right) \in \mathbb{A}_{k}^{n}$ qualquer é um conjunto algébrico afim. Mais precisamente, $\{P\}=\mathcal{Z}\left(x_{1}-p_{1}, \ldots, x_{n}-p_{n}\right)$.

É fácil observar que a aplicação $\mathcal{Z}$ inverte inclusões, isto é, se $S \subset S^{\prime}$, então $\mathcal{Z}\left(S^{\prime}\right) \subset \mathcal{Z}(S)$.

Cabe aqui observar que se $I=\langle S\rangle \subset k\left[x_{1}, x_{2}, \ldots, x_{n}\right]$ é o ideal gerado por $S$, então $\mathcal{Z}(I)=\mathcal{Z}(S)$. Como $k\left[x_{1}, x_{2}, \ldots, x_{n}\right]$ é um anel noetheriano, então para todo conjunto algébrico $Z$ de $\mathbb{A}_{k}^{n}$ existe um ideal $I \subset k\left[x_{1}, x_{2}, \ldots, x_{n}\right]$ tal que $Z=\mathcal{Z}(I)$. 
Esse ideal não é sempre único. Por exemplo, quando $k=\mathbb{R}$ e $n=2$, temos uma família de polinômios $f_{i}=x_{1}^{2 i}+x_{2}^{2 i} \operatorname{com} \mathcal{Z}\left(\left\langle f_{i}\right\rangle\right)=\mathcal{Z}\left(f_{i}\right)=\{(0,0)\}$. Ou seja, diferentes ideais definem o mesmo conjunto algébrico afim.

Seja $I \subset k\left[x_{1}, x_{2}, \ldots, x_{n}\right]$ um ideal. Podemos definir o radical de $I$ como

$$
\sqrt{I}=\left\{f \in k\left[x_{1}, x_{2}, \ldots, x_{n}\right] \mid \exists m \in \mathbb{N}^{*} f^{m} \in I\right\},
$$

e dizemos que um ideal $I$ é um ideal radical quando $\sqrt{I}=I$.

É fácil ver que para qualquer ideal $I \subset k\left[x_{1}, x_{2}, \ldots, x_{n}\right]$, vale o seguinte:

(i) $\sqrt{I}$ é um ideal, e mais ainda, um ideal radical;

(ii) $\mathcal{Z}(\sqrt{I})=\mathcal{Z}(I)$.

Por esses motivos, se $Z \subset \mathbb{A}_{k}^{n}$ é um conjunto algébrico afim e $I \subset$ $k\left[x_{1}, x_{2}, \ldots, x_{n}\right]$ é um ideal tal que $Z=\mathcal{Z}(I)$, então o ideal radical $I^{\prime}=\sqrt{I}$ satisfaz $Z=\mathcal{Z}\left(I^{\prime}\right)$. Isso mostra que sempre podemos escolher um ideal radical $I \operatorname{com} Z=\mathcal{Z}(I)$.

Com o Teorema de Zeros de Hilbert, enunciado a seguir, podemos ver que, se $k$ é algebricamente fechado, então esse ideal radical é único, e mais ainda, podemos construí-lo a partir do conjunto algébrico afim. Dado $Z \subset \mathbb{A}_{k}^{n}$ um conjunto algébrico, o conjunto de polinômios

$$
\mathcal{I}(Z)=\left\{f \in k\left[x_{1}, x_{2}, \ldots, x_{n}\right] \mid f(P)=0 \forall P \in Z\right\}
$$

é chamado o ideal de $Z$, e é um ideal radical.

É fácil observar que $\mathcal{I}$ também inverte inclusões, isto é, se $Z \subset Z^{\prime}$ são conjuntos algébricos de $\mathbb{A}_{k}^{n}$, então $\mathcal{I}\left(Z^{\prime}\right) \subset \mathcal{I}(Z)$.

Teorema 2.1 (O Teorema dos Zeros de Hilbert). Seja $k$ um corpo algebricamente fechado, $I \subset k\left[x_{1}, x_{2}, \ldots, x_{n}\right]$ um ideal e $f \in k\left[x_{1}, x_{2}, \ldots, x_{n}\right]$ um polinômio. Então

$$
f(P)=0 \forall P \in \mathcal{Z}(I) \Leftrightarrow f \in \sqrt{I} .
$$

Ou equivalentemente,

$$
\mathcal{I}(\mathcal{Z}(I))=\sqrt{I}
$$

(Para referência, consultar (LANG, 2002) p. 378)

Como consequência, se $I$ é um ideal radical, então $\mathcal{I}(\mathcal{Z}(I))=I$. Isso mostra que $\mathcal{Z}$ e $\mathcal{I}$ são inversas, e constituem uma bijeção entre conjuntos algébricos de $\mathbb{A}_{k}^{n}$ e ideais radicais de $k\left[x_{1}, x_{2}, \ldots, x_{n}\right]$. Essa dualidade nos permite relacionar propriedades geométricas de um conjunto algébrico afim com propriedades algébricas do seu ideal radical.

Exemplo 3. Se $k$ é algebricamente fechado, pontos de $\mathbb{A}_{k}^{n}$ estão em bijeção com ideais maximais de $k\left[x_{1}, x_{2}, \ldots, x_{n}\right]$. De fato, um ponto não possui 
subconjunto algébrico próprio não vazio, então seu ideal não pode estar propriamente contido em um ideal diferente de $\mathcal{I}(\varnothing)=k\left[x_{1}, x_{2}, \ldots, x_{n}\right]$. Como pontos são os únicos conjuntos algébricos afins com essa propriedade, a recíproca também vale. Assim, ideais maximais de $k\left[x_{1}, x_{2}, \ldots, x_{n}\right]$ são da forma $\left\langle x_{1}-p_{1}, \ldots, x_{n}-p_{n}\right\rangle$.

Definimos o anel de coordenadas de um conjunto algébrico $Z \subset \mathbb{A}^{n}$ como $\mathcal{A}(Z)=k\left[x_{1}, x_{2}, \ldots, x_{n}\right] / \mathcal{I}(Z)$. Quando $Z \neq \varnothing, \mathcal{A}(Z)$ é uma $k$-álgebra finitamente gerada sem nilpotentes.

O homomorfismo sobrejetor natural de $k\left[x_{1}, x_{2}, \ldots, x_{n}\right]$ em $\mathcal{A}(Z)$ induz uma bijeção entre ideais de $k\left[x_{1}, x_{2}, \ldots, x_{n}\right]$ que contém $\mathcal{I}(Z)$ e ideais de $\mathcal{A}(Z)$. Essa bijeção preserva ideais radicais, portanto, quando $k$ é algebricamente fechado, subconjuntos algébricos de $Z$ estão em bijeção com ideais radicais de $\mathcal{A}(Z)$.

\section{2}

\section{O espaço projetivo e conjuntos algébricos projetivos}

O espaço projetivo de dimensão $n$ sobre um corpo $k$ é definido como o conjunto de retas em $k^{n+1}$ passando pela origem. Algebricamente, podemos escrever

$$
\mathbb{P}_{k}^{n}=\frac{k^{n+1} \backslash\{0\}}{\sim}
$$

com a seguinte relação de equivalência: dados $v$ e $w$ em $k^{n+1} \backslash\{0\}$, então $v \sim w$ se existe $\lambda \in k \backslash\{0\}$ tal que $v=\lambda w$, que é equivalente a dizer que $v$ e $w$ estão na mesma reta passando pela origem.

Um ponto $P$ de $\mathbb{P}^{n}$ pode ser escrito em coordenadas homogêneas tomando-se um representante $\left(p_{0}, p_{1}, \ldots, p_{n}\right) \in k^{n+1}$ como $P=\left(p_{0}: p_{1}: \ldots: p_{n}\right)$. Em coordenadas homogêneas, $P$ pode ter diferentes representações. Mais precisamente, $\left(p_{0}: p_{1}: \ldots: p_{n}\right)=\left(q_{0}: q_{1}: \ldots: q_{n}\right)$ se e somente se existe $\lambda \in k \backslash\{0\}$ tal que $p_{i}=\lambda q_{i}$ para todo $i=0,1, \ldots, n$.

Nosso objetivo a seguir será introduzir os conjuntos algébricos projetivos como conjuntos de zeros de polinômios. Porém, no caso projetivo, dado $f \in k\left[x_{0}, x_{1}, \ldots, x_{n}\right], f(P)$ depende da escolha de coordenadas homogêneas de $P$, ou seja, não podemos avaliar $f$ em $P$.

Apesar disso, é possível decidir se $F(P)=0$ se $F \in k\left[x_{0}, x_{1}, \ldots, x_{n}\right]^{h}$ for um polinômio homogêneo. De fato, nesse caso, se $F\left(p_{0}, p_{1}, \ldots, p_{n}\right)=0$ para alguma escolha de coordenadas homogêneas de $P$, então para todo $\lambda \in k \backslash\{0\}$, vale $F\left(\lambda p_{0}, \lambda p_{1}, \ldots, \lambda p_{n}\right)=\lambda^{\operatorname{deg}(F)} F\left(p_{0}, p_{1}, \ldots, p_{n}\right)=0$, o que significa que $F$ se anula na reta que passa pela origem e pelo ponto $\left(p_{0}, p_{1}, \ldots, p_{n}\right)$. 
Sendo assim, o conjunto

$$
\mathcal{Z}(F)=\left\{P \in \mathbb{P}_{k}^{n} \mid F(P)=0\right\}
$$

está bem definido e é chamado o conjunto dos zeros de $F$.

Mais geralmente, seja $S \subset k\left[x_{0}, x_{1}, \ldots, x_{n}\right]^{h}$ um conjunto de polinômios homogêneos, podemos definir o conjunto de zeros de $S$ como

$$
\mathcal{Z}(S)=\left\{P \in \mathbb{P}_{k}^{n} \mid F(P)=0 \forall F \in S\right\}
$$

Tais subconjuntos de $\mathbb{P}_{k}^{n}$ são chamados de conjuntos algébricos projetivos ou conjuntos algébricos de $\mathbb{P}_{k}^{n}$.

Exemplo 4. Tanto $\mathbb{P}_{k}^{n}$ quanto $\varnothing$ são conjuntos algébrico projetivos, pois são os conjuntos de zeros dos polinômios homogêneos $F=0$ e $G=1$ respectivamente. Exemplo 5. Pontos de $\mathbb{P}_{k}^{n}$ também são conjuntos algébricos projetivos. De fato, se $P=\left(p_{0}: p_{1}: \ldots: p_{n}\right)$ e supondo sem perdas que $p_{0} \neq 0$, então $\{P\}=$ $\mathcal{Z}\left(\left\{p_{0} x_{i}-p_{i} x_{0} \mid i=1,2, \ldots, n\right\}\right)$.

Essa definição também pode ser estendida para qualquer subconjunto de polinômios: seja $f \in k\left[x_{0}, x_{1}, \ldots, x_{n}\right]$ um polinômio qualquer, podemos dizer que $f(P)=0$ quando $f\left(p_{0}, p_{1}, \ldots, p_{n}\right)=0$ para qualquer escolha de coordenadas homogêneas de $P$. Ao fixar uma escolha de coordenadas $\left(p_{0}, p_{1}, \ldots, p_{n}\right)$ e escrevendo $f=\sum_{i=0}^{n} F_{i}$ como soma de seus termos homogêneos, com $F_{i}$ sendo seu termo de grau $i$, então

$$
f\left(\lambda p_{0}, \lambda p_{1}, \ldots, \lambda p_{n}\right)=\sum_{i=1}^{n} \lambda^{i} F_{i}\left(p_{0}, p_{1}, \ldots, p_{n}\right)
$$

é um polinômio em $\lambda$, que é identicamente nulo se e só se cada $F_{i}$ se anular em $P$.

Então, seja $S \subset k\left[x_{0}, x_{1}, \ldots, x_{n}\right]$ um subconjunto qualquer de polinômios, podemos definir o conjunto de zeros de $S$ como

$$
\mathcal{Z}(S)=\left\{P \in \mathbb{P}_{k}^{n} \mid f(P)=0 \forall f \in S\right\}
$$

É fácil observar que tal aplicação inverte inclusões, isto é, se $S \subset S^{\prime}$, então $\mathcal{Z}\left(S^{\prime}\right) \subset \mathcal{Z}(S)$.

Seja $S \subset k\left[x_{0}, x_{1}, \ldots, x_{n}\right]^{h}$ um conjunto de polinômios homogêneos e $I=\langle S\rangle \subset k\left[x_{0}, x_{1}, \ldots, x_{n}\right]$ o ideal gerado por $S$. Dizemos que $I$ é um ideal homogêneo, pois é gerado por um conjunto de polinômios homogêneos, e vale que $\mathcal{Z}(I)=\mathcal{Z}(S)$. 
Também podemos obter ideais homogêneos a partir de conjuntos algébricos: seja $Z \subset \mathbb{P}_{k}^{n}$ um conjunto algébrico, definimos

$$
\mathcal{I}(Z)=\left\langle F \in k\left[x_{0}, x_{1}, \ldots, x_{n}\right]^{h} \mid F(P)=0 \forall P \in Z\right\rangle
$$

o ideal homogêneo de $Z$.

É fácil observar que $\mathcal{I}$ inverte inclusões, isto é, se $Z \subset Z^{\prime}$ são conjuntos algébricos projetivos, então $\mathcal{I}\left(Z^{\prime}\right) \subset \mathcal{I}(Z)$.

A versão projetiva do Teorema dos Zeros de Hilbert pode nos esclarecer em que condições as aplicações $\mathcal{Z}$ e $\mathcal{I}$ são inversas, e é uma consequência fácil do Teorema 2.1. Antes de enunciá-la, relembramos que o radical de um ideal $I$ é definido como

$$
\sqrt{I}=\left\{f \in k\left[x_{0}, x_{1}, \ldots, x_{n}\right] \mid \exists m \in \mathbb{N}^{*} f^{m} \in I\right\} .
$$

Teorema 2.2 (O Teorema dos Zeros de Hilbert Projetivo). Seja $k$ um corpo algebricamente fechado, $I \subset k\left[x_{0}, x_{1}, \ldots, x_{n}\right]$ um ideal homogêneo tal que $\sqrt{I} \neq\left\langle x_{0}, x_{1}, \ldots, x_{n}\right\rangle$ e $F \in k\left[x_{0}, x_{1}, \ldots, x_{n}\right]^{h}$ um polinômio homogêneo. Então

$$
F(P)=0 \forall P \in \mathcal{Z}(I) \Leftrightarrow F \in \sqrt{I}
$$

Podemos concluir que se $k$ é algebricamente fechado e $I \subset k\left[x_{0}, x_{1}, \ldots, x_{n}\right]$ é um ideal homogêneo tal que $\sqrt{I} \neq\left\langle x_{0}, x_{1}, \ldots, x_{n}\right\rangle$, então

$$
\mathcal{I}(\mathcal{Z}(I))=\sqrt{I}
$$

Quando $I$ é um ideal homogêneo radical, isto é, um ideal homogêneo tal que $I=\sqrt{I}$, então as aplicações $\mathcal{Z}$ e $\mathcal{I}$ são inversas, e nos dão uma bijeção entre os conjuntos algébricos projetivos de $\mathbb{P}^{n}$ e os ideais homogêneos radicais de $k\left[x_{0}, x_{1}, \ldots, x_{n}\right]$ diferentes de $\left\langle x_{0}, x_{1}, \ldots, x_{n}\right\rangle$.

Definimos o anel de coordenadas homogêneas de um conjunto algébrico $Z \subset \mathbb{P}^{n}$ como $S(Z)=k\left[x_{0}, x_{1}, \ldots, x_{n}\right] / \mathcal{I}(Z)$, que é uma $k$-álgebra finitamente gerada e sem nilpotentes. Analogamente ao caso afim, quando $k$ é algebricamente fechado, subconjuntos algébricos de $Z$ estão em bijeção com ideais radicais de $S(Z)$ diferentes da classe de $\left\langle x_{0}, x_{1}, \ldots, x_{n}\right\rangle$ em $S(Z)$.

A partir de agora até o final desta dissertação, a menos que se explicite o contrário, vamos considerar $k$ um corpo algebricamente fechado. 


\section{3}

\section{A topologia de Zariski}

Sabemos que $\varnothing$ e $\mathbb{A}_{k}^{n}$ são conjuntos algébricos afins. Além disso, a união de dois conjuntos algébricos afins $\mathcal{Z}(I) \cup \mathcal{Z}(J)$ é também um conjunto algébrico afim $\mathcal{Z}(I \cdot J)$ e a interseção arbitrária de conjuntos algébricos afins $\cap \mathcal{Z}\left(I_{\alpha}\right)$ é um conjunto algébrico afim $\mathcal{Z}\left(\left\langle I_{\alpha}\right\rangle\right)$. Com isso, podemos definir uma topologia em $\mathbb{A}_{k}^{n}$ onde os conjuntos fechados são os conjuntos algébricos afins. Tal topologia é chamada de Topologia de Zariski em $\mathrm{A}_{k}^{n}$.

Exemplo 6. Em $\mathbb{A}_{k}^{1}$, os únicos conjuntos fechados são o próprio $\mathbb{A}_{k}^{1}$ e subconjuntos finitos. De fato, se o conjunto algébrico é definido por pelo menos um polinômio não nulo, possui finitos pontos. Reciprocamente, qualquer conjunto finito de pontos em $\mathrm{A}_{k}^{1}$ é o conjunto de zeros de algum polinômio. Observe que esta topologia não é Hausdorff.

De forma análoga, podemos definir uma topologia (a topologia de Zariski em $\mathbb{P}_{k}^{n}$ ) no espaço projetivo, onde os conjuntos fechados são os conjuntos algébricos projetivos.

Dizemos que um subconjunto não vazio $X$ de um espaço topológico é redutível quando existem subconjuntos próprios $A, B \subset X$ fechados em $X$ tais que $X=A \cup B$. Dizemos que $X$ é irredutível caso contrário. O conjunto vazio é considerado redutível.

Exemplo 7. Qualquer ponto de $\mathbb{A}_{k}^{n}$ ou $\mathbb{P}_{k}^{n}$ é irredutível. O espaço afim $\mathbb{A}_{k}^{1}$ também é irredutível, pois todo subconjunto próprio fechado de $\mathbb{A}_{k}^{1}$ é finito, enquanto que $\mathbb{A}_{k}^{1}=k$ é infinito por ser algebricamente fechado.

Exemplo 8. O conjunto $\mathcal{Z}\left(x_{1}^{2}-x_{2}^{2}\right)$ em $\mathbb{A}_{k}^{2}$ ou em $\mathbb{P}_{k}^{2}$ é redutível, pois equivale à união $\mathcal{Z}\left(x_{1}-x_{2}\right) \cup \mathcal{Z}\left(x_{1}+x_{2}\right)$.

Proposição 2.1. Um conjunto algébrico $Z$ é irredutivel se e só se $\mathcal{I}(Z)$ é um ideal primo.

Prova. Os casos afim e projetivo são análogos. Vamos focar no caso afim e provar os dois lados por contraposição.

Suponha que $Z \neq \varnothing$ é um conjunto algébrico afim redutível. Então existem conjuntos algébricos próprios $Z_{1} \subsetneq Z$ e $Z_{2} \subsetneq Z$ tais que $Z=Z_{1} \cup Z_{2}$. Em particular, temos que

$$
\mathcal{I}(Z) \subsetneq \mathcal{I}\left(Z_{i}\right)
$$

Sejam $f_{i} \in \mathcal{I}\left(Z_{i}\right) \backslash \mathcal{I}(Z)$. Então é fácil ver que $f_{1} \cdot f_{2} \in \mathcal{I}(Z)$, e portanto $\mathcal{I}(Z)$ não é um ideal primo. 
Suponha agora que $Z \neq \varnothing$ é um conjunto algébrico afim tal que $I=\mathcal{I}(Z)$ não é um ideal primo. Então existem $f_{1}, f_{2} \notin I$ tais que $f_{1} \cdot f_{2} \in I$. Sejam

$$
Z_{i}=\mathcal{Z}\left(\left\langle f_{i}, I\right\rangle\right) \subset Z
$$

então $Z=Z_{1} \cup Z_{2}$, pois qualquer ponto $P \in Z$ satisfaz que $f_{1} \cdot f_{2}(P)=0$, e sem perdas podemos assumir que $f_{1}(P)=0$. Mas como $f(P)=0$ para todo $f \in I$, temos que $P \in Z_{1}$. Então $Z$ é redutível.

Quando $Z=\varnothing$, temos que $\mathcal{I}(Z)=k\left[x_{1}, \ldots, x_{n}\right]$ é todo o anel e não é primo, o que condiz com a convenção de que $\varnothing$ é redutível.

Exemplo 9. O espaço afim $\mathbb{A}_{k}^{n}$ é irredutível, afinal $\mathcal{I}\left(\mathbb{A}_{k}^{n}\right)=\{0\}$ é um ideal primo.

Como consequência, podemos inferir que um conjunto algébrico $Z$ é irredutível se e só se $\mathcal{A}(Z)$ (ou $S(Z)$ dependendo se $Z$ é afim ou projetivo) é um domínio de integridade.

Uma variedade algébrica afim é um fechado irredutível de $\mathbb{A}_{k}^{n}$ e uma variedade algébrica projetiva é um fechado irredutível de $\mathbb{P}_{k}^{n}$. Também podemos definir uma topologia em qualquer subconjunto de $\mathbb{A}_{k}^{n}$ (ou $\mathbb{P}_{k}^{n}$ ), induzida pela topologia de Zariski em $\mathbb{A}_{k}^{n}\left(\right.$ ou $\left.\mathbb{P}_{k}^{n}\right)$.

Uma variedade quasi-afim (quasi-projetiva) é um aberto $A \subset X$ de uma variedade afim (projetiva) $X$.

Proposição 2.2. Se $A \neq \varnothing$ é um aberto da variedade algébrica $X$ (afim ou projetiva), então $A$ é denso em $X$ e irredutivel.

Prova. Dado $Y \subset X$, seja $\bar{Y}$ o fecho topológico de $Y$ em $X$, isto é, a interseção de todos os conjuntos fechados em $X$ que contêm $Y$.

Se $\bar{A} \neq X$, então $X$ seria redutível, pois poderíamos expressar $X$ como uma união de subconjuntos algébricos próprios: $X=\bar{A} \cup(X \backslash A)$, e portanto $\bar{A}=X$

Além disso, se $A=A_{1} \cup A_{2}$ é uma união de fechados de $A$, então $X=\overline{A_{1} \cup A_{2}} \subset \overline{A_{1}} \cup \overline{A_{2}}$. Assim, $X=\overline{A_{1}} \cup \overline{A_{2}}$, e por ser irredutível, podemos supor sem perdas que $X=\overline{A_{1}}$. Como $A_{1}$ é um fechado de $A$, sabemos que $A_{1}=\overline{A_{1}} \cap A=A$. Portanto $A_{1}$ não é um fechado próprio de $A$, e por isso, $A$ é irredutível.

O mesmo vale para abertos não vazios de variedades quasi-projetivas, pois se $U \subset A$ é um aberto não vazio da variedade quasi-projetiva $A$, que por sua vez é um aberto da variedade projetiva $X$, então $U \subset X$ é um aberto não vazio de $X$, e portanto denso em $X$ (e consequentemente em A) e irredutível. 
Proposição 2.3. Com essas topologias em $\mathbb{A}_{k}^{n}$ e $\mathcal{U}_{0}=\mathbb{P}_{k}^{n} \backslash \mathcal{Z}\left(x_{0}\right)$, o mapa $\varphi_{0}: \mathbb{A}_{k}^{n} \rightarrow \mathcal{U}_{0} ;\left(p_{1}, \ldots, p_{n}\right) \mapsto\left(1: p_{1}: \ldots: p_{n}\right)$ é um homeomorfismo com inversa $\psi_{0}: \mathcal{U}_{0} \rightarrow \mathbb{A}_{k}^{n} ;\left(q_{0}: q_{1}: \ldots: q_{n}\right) \mapsto\left(\frac{q_{1}}{q_{0}}, \ldots, \frac{q_{n}}{q_{0}}\right)$.

Prova. É fácil ver que as aplicações são de fato inversas, e portanto são bijeções. Para verificar que $\varphi_{0}$ é um homeomorfismo, basta mostrar que $\psi_{0}$ e $\varphi_{0}$ levam fechados em fechados, o que implicaria que $\varphi_{0}$ e $\psi_{0}$, respectivamente, são contínuas.

Seja $Y$ um subconjunto fechado de $\mathcal{U}_{0}$ e $\bar{Y}$ seu fecho em $\mathbb{P}_{k}^{n}$. Seja $I=\mathcal{I}(\bar{Y}) \subset k\left[x_{0}, x_{1}, \ldots, x_{n}\right]$ seu ideal homogêneo radical. Sabemos que $I$ possui uma base de polinômios homogêneos, e pelo Teorema das bases de Hilbert, $I$ pode ser gerado por finitos deles, que chamaremos de $F_{i} \subset k\left[x_{0}, x_{1}, \ldots, x_{n}\right]^{h}$, $i=1, \ldots, l$.

Para cada $i$, seja $f_{i}=F_{i}\left(1, x_{1}, \ldots, x_{n}\right) \in k\left[x_{1}, \ldots, x_{n}\right]$ a desomogeneização de $F_{i}$ na primeira coordenada. Seja $I^{\prime}=\left\langle f_{i}\right\rangle \subset k\left[x_{1}, \ldots, x_{n}\right]$ o ideal gerado por tais desomogeneizações. Assim, $\psi_{0}(Y)=\mathcal{Z}\left(I^{\prime}\right) \subset \mathbb{A}_{k}^{n}$ e portanto é um fechado de $A_{k}^{n}$.

Reciprocamente, seja $Z$ um fechado de $\mathbb{A}_{k}^{n}$ e $I=\mathcal{I}(Z) \subset k\left[x_{1}, \ldots, x_{n}\right]$ seu ideal radical. Novamente pelo Teorema das bases de Hilbert, $I$ é gerado por finitos polinômios, chamados $f_{i} \subset k\left[x_{1}, \ldots, x_{n}\right], i=1, \ldots, l$.

Para cada $i$, seja $F_{i}\left(x_{0}, x_{1}, \ldots, x_{n}\right)=x_{0}^{d_{i}} f_{i}\left(\frac{x_{1}}{x_{0}}, \ldots, \frac{x_{n}}{x_{0}}\right) \in k\left[x_{0}, x_{1}, \ldots, x_{n}\right]$ a homogeneização de $f_{i}$, onde $d_{i}=\operatorname{deg}\left(f_{i}\right)$. Seja $\widetilde{I}=\left\langle F_{i}\right\rangle \subset k\left[x_{0}, x_{1}, \ldots, x_{n}\right]$ o ideal homogêneo gerado pelas homogeneizações. Então $\varphi_{0}(Z)=\mathcal{U}_{0} \cap \mathcal{Z}(\widetilde{I})$ é um fechado de $\mathcal{U}_{0}$.

Por meio desse homeomorfismo, qualquer aberto de uma variedade afim (isto é, uma variedade quasi-afim) pode ser visto como uma variedade quasiprojetiva.

Além disso, usando homeomorfismos $\varphi_{i}: \mathbb{A}_{k}^{n} \rightarrow \mathcal{U}_{i}$ semelhantes a $\varphi_{0}$, onde $\mathcal{U}_{i}=\mathbb{P}_{k}^{n} \backslash \mathcal{Z}\left(x_{i}\right)$, podemos cobrir $\mathbb{P}_{k}^{n}$ com abertos $\mathcal{U}_{i}$ homeomorfos a $\mathbb{A}_{k}^{n}$.

\section{4}

\section{$\mathrm{O}$ produto cartesiano de variedades algébricas}

Dadas duas variedades algébricas $X$ e $Y$, cada uma afim ou projetiva, nosso objetivo é definir uma estrutura de variedade para o produto cartesiano $X \times Y$.

Se ambas forem variedades algébricas afins, com $X \subset \mathbb{A}_{k}^{n}$ e $Y \subset \mathbb{A}_{k}^{m}$, podemos aproveitar a bijeção natural entre $\mathbb{A}_{k}^{n} \times \mathbb{A}_{k}^{m}$ e $\mathbb{A}_{k}^{n+m}$, definida por

$$
\left(\left(a_{1}, \ldots, a_{n}\right),\left(b_{1}, \ldots, b_{m}\right)\right) \mapsto\left(a_{1}, \ldots, a_{n}, b_{1}, \ldots, b_{m}\right)
$$


Usando a topologia de Zariski em $\mathbb{A}_{k}^{n+m}$, podemos dar a $\mathbb{A}_{k}^{n} \times \mathbb{A}_{k}^{m}$ uma estrutura de variedade afim. Essa topologia é diferente da topologia produto.

Sejam $\pi_{1}$ e $\pi_{2}$ as respectivas projeções naturais de $\mathbb{A}_{k}^{n+m}$ em $\mathbb{A}_{k}^{n}$ e $\mathbb{A}_{k}^{m}$ pela bijeção acima, então o produto cartesiano

$$
X \times Y \cong \mathcal{Z}\left(\left\{f \circ \pi_{1}\right\}_{f \in \mathcal{I}(X)} \cup\left\{g \circ \pi_{2}\right\}_{g \in \mathcal{I}(Y)}\right) \subset \mathbb{A}_{k}^{n+m}
$$

pode ser visto como uma variedade afim.

Para obter uma construção análoga para variedades projetivas, precisamos de uma bijeção entre $\mathbb{P}_{k}^{n} \times \mathbb{P}_{k}^{m}$ e uma variedade projetiva. Para isso definiremos o mergulho de Segre $\mathscr{S}: \mathbb{P}_{k}^{n} \times \mathbb{P}_{k}^{m} \rightarrow \mathbb{P}_{k}^{N}$, onde $N=(n+1)(m+1)-1$, por

$$
\left(\left(a_{0}: \ldots: a_{n}\right),\left(b_{0}: \ldots: b_{m}\right)\right) \mapsto\left(c_{00}: \ldots: c_{0 m}: c_{10}: \ldots: c_{1 m}: \ldots: c_{n m}\right),
$$

onde $c_{i j}=a_{i} b_{j}$, com $i=0,1, \ldots, n$ e $j=0,1, \ldots, m$.

Note que outra escolha de coordenadas homogêneas para $\left(a_{0}: \ldots: a_{n}\right)$ ou $\left(b_{0}: \ldots: b_{m}\right)$ leva ao mesmo ponto na imagem. Além disso, alguma coordenada homogênea $a_{s}$ é não nula, assim como alguma coordenada $b_{t}$ e portanto $c_{s t}$ é uma coordenada não nula da imagem. Isso significa que o mapa $\mathscr{S}$ está bem definido.

Também é possível notar que $\mathscr{S}$ é injetiva, pois se $\left(c_{00}: \ldots: c_{n m}\right)$ pertence à imagem com coordenada $c_{s t}$ não nula para certos $s, t$, então $a_{s}, b_{t} \neq 0$ e necessariamente $\left(a_{0}: a_{1}: \ldots: a_{m}\right)=\left(a_{0} b_{t}: a_{1} b_{t}: \ldots: a_{n} b_{t}\right)=\left(c_{0 t}: c_{1 t} \ldots: c_{n t}\right) \mathrm{e}$ $\left(b_{0}: b_{1}: \ldots: b_{m}\right)=\left(a_{s} b_{0}: a_{s} b_{1}: \ldots: a_{s} b_{m}\right)=\left(c_{s 0}: c_{s 1} \ldots: c_{s m}\right)$ são encontrados de forma única.

Nosso objetivo é mostrar que $\mathscr{S}\left(\mathbb{P}_{k}^{n} \times \mathbb{P}_{k}^{m}\right)$ é um fechado de $\mathbb{P}_{k}^{N}$, e portanto $\mathbb{P}_{k}^{n} \times \mathbb{P}_{k}^{m}$ está em bijeção com uma variedade projetiva em $\mathbb{P}_{k}^{N}$, e poderemos dar ao produto cartesiano uma estrutura de variedade.

Seja $J=\left\langle z_{i j} z_{s t}-z_{i t} z_{s j}\right\rangle \subset k\left[z_{00}, \ldots, z_{n m}\right]$, com $i, s=0,1, \ldots, n$ e $j, t=$ $0,1, \ldots, m$. É claro que $\mathscr{S}\left(\mathbb{P}_{k}^{n} \times \mathbb{P}_{k}^{m}\right) \subset \mathcal{Z}(J) \subset \mathbb{P}_{k}^{N}$, afinal para todo $\left(c_{i j}\right)=$ $\mathscr{S}\left(\left(a_{i}\right),\left(b_{j}\right)\right)$, temos $c_{i j} c_{s t}=a_{i} b_{j} a_{s} b_{t}=a_{i} b_{t} a_{s} b_{j}=c_{i t} c_{s j}$. Então basta mostrar que para cada ponto $R=\left(r_{i j}\right) \in \mathcal{Z}(J)$ existe $(P, Q) \in \mathbb{P}_{k}^{n} \times \mathbb{P}_{k}^{m}$ tal que $\mathscr{S}(P, Q)=R$.

Fixe $s, t$ tais que $r_{s t} \neq 0$ e defina $P=\left(r_{0 t}: r_{1 t}: \ldots: r_{n t}\right)$ e $Q=$ $\left(r_{s 0}: r_{s 1}: \ldots: r_{s m}\right)$. Então $\mathscr{S}(P, Q)=\left(c_{i j}\right), \operatorname{com} c_{i j}=r_{i t} r_{s j}=r_{i j} r_{s t}$, logo $\mathscr{S}(P, Q)=\left(c_{i j}\right)=\left(r_{i j}\right)$, como queríamos.

Com isso, podemos identificar $\mathbb{P}_{k}^{n} \times \mathbb{P}_{k}^{m}$ com a variedade projetiva $\mathscr{S}\left(\mathbb{P}_{k}^{n} \times \mathbb{P}_{k}^{m}\right)=\mathcal{Z}(J) \subset \mathbb{P}_{k}^{N}$ junto com a sua estrutura topológica, que será diferente da topologia produto para $\mathbb{P}_{k}^{n} \times \mathbb{P}_{k}^{m}$. A partir de agora, usare$\operatorname{mos}\left(a_{0}: \ldots: a_{n} ; b_{0}: \ldots: b_{m}\right)$ para denotar o ponto $\mathscr{S}\left(\left(a_{0}: \ldots: a_{n}\right),\left(b_{0}: \ldots: b_{m}\right)\right) \epsilon$ 
$\mathscr{S}\left(\mathbb{P}_{k}^{n} \times \mathbb{P}_{k}^{m}\right)$, mas por abuso de notação escrevemos $\left(a_{0}: \ldots: a_{n} ; b_{0}: \ldots: b_{m}\right) \in$ $\mathbb{P}_{k}^{n} \times \mathbb{P}_{k}^{m}$.

Exemplo 10. O produto $\mathbb{P}_{k}^{1} \times \mathbb{P}_{k}^{1}$ pode ser mergulhado em $\mathbb{P}_{k}^{3}$ como a variedade projetiva $\mathcal{Z}\left(z_{00} z_{11}-z_{01} z_{10}\right)$. Vamos ver que as fibras do mapa de projeção $\pi: \mathscr{S}\left(\mathbb{P}_{k}^{1} \times \mathbb{P}_{k}^{1}\right) \rightarrow \mathbb{P}_{k}^{1}$ tal que $\left(a_{0}: a_{1} ; b_{0}: b_{1}\right) \mapsto\left(a_{0}: a_{1}\right)$ são retas em $\mathbb{P}_{k}^{3}$.

Seja $P=\left(p_{0}: p_{1}\right) \in \mathbb{P}_{k}^{1}$, temos

$$
\pi^{-1}(P)=\left\{\left(p_{0} b_{0}: p_{0} b_{1}: p_{1} b_{0}: p_{1} b_{1}\right) \in \mathbb{P}_{k}^{3} \mid\left(b_{0}: b_{1}\right) \in \mathbb{P}_{k}^{1}\right\}
$$

e portanto $\pi^{-1}(P) \subset \mathcal{Z}\left(p_{0} z_{10}-p_{1} z_{00}, p_{0} z_{11}-p_{1} z_{01}\right) \subset \mathbb{P}_{k}^{3}$. Porém, dado $R=$ $\left(r_{00}: r_{01}: r_{10}: r_{11}\right) \in \mathcal{Z}\left(p_{0} z_{10}-p_{1} z_{00}, p_{0} z_{11}-p_{1} z_{01}\right)$, podemos supor sem perdas $p_{0} \neq 0$, então $Q=\left(r_{00}: r_{01}\right)$ nos garante que $\mathscr{S}(P, Q)=R$, logo

$$
\pi^{-1}(P)=\mathcal{Z}\left(p_{0} z_{10}-p_{1} z_{00}, p_{0} z_{11}-p_{1} z_{01}\right) \subset \mathbb{P}_{k}^{3}
$$

A partir de agora, vamos descrever os fechados de $\mathbb{P}_{k}^{n} \times \mathbb{P}_{k}^{m}$ em termos das coordenadas de $\mathbb{P}_{k}^{n}$ e $\mathbb{P}_{k}^{m}$.

Os fechados de $\mathbb{P}_{k}^{n} \times \mathbb{P}_{k}^{m}$ serão os subconjuntos $\mathscr{S}^{-1}(W)$, onde $W \subset \mathbb{P}_{k}^{N}$ é um fechado. Pelo Teorema das Bases de Hilbert, sabemos que $\mathcal{I}(W)$ é gerado por finitos polinômios homogêneos $F_{s} \in k\left[z_{00}, \ldots, z_{n m}\right]^{h}, s=1, \ldots, l$. Então dados $P=\left(p_{0}: \ldots: p_{n}\right) \in \mathbb{P}_{k}^{n}$ e $Q=\left(q_{0}: \ldots: q_{m}\right) \in \mathbb{P}_{k}^{m}$,

$$
\mathscr{S}(P, Q) \in W \Leftrightarrow F_{s}\left(p_{i} q_{j}\right)=0 \forall s=1, \ldots, l
$$

Considere $G_{s}=F_{s}\left(x_{i} y_{j}\right) \in k\left[x_{0}, \ldots, x_{n}, y_{0}, \ldots, y_{m}\right]$ para todo $s=1, \ldots, l$. Então cada $G_{s}$ é homogêneo de grau $\operatorname{deg}\left(F_{s}\right)$ nas coordenadas $x_{i}$ e também nas coordenadas $y_{j}$. Neste caso, dizemos que $G_{s}$ é bi-homogêneo, e tem bigrau $\left(\operatorname{deg}\left(F_{s}\right), \operatorname{deg}\left(F_{s}\right)\right)$. Em geral, um polinômio bi-homogêneo pode ter bigrau $(a, b)$ com $a \neq b$. Reciprocamente, se $G_{s} \in k\left[x_{0}, \ldots, x_{n}, y_{0}, \ldots, y_{m}\right]$ é um polinômio bi-homogêneo de bi-grau $\left(d_{s}, d_{s}\right)$, não é difícil perceber que existe $F_{s} \in k\left[z_{00}, \ldots, z_{n m}\right]^{h}$ de grau $d_{s}$ tal que $G_{s}=F_{s}\left(x_{i} y_{j}\right)$.

Note que se $G_{s} \in k\left[x_{0}, \ldots, x_{n}, y_{0}, \ldots, y_{m}\right]$ são polinômios bi-homogêneos com $s=1, \ldots, l$, podemos definir

$$
\mathcal{Z}\left(G_{1}, \ldots, G_{l}\right)=\left\{(P, Q) \in \mathbb{P}_{k}^{n} \times \mathbb{P}_{k}^{m} \mid G_{s}(P, Q)=0 \forall s=1, \ldots, l\right\}
$$

Podemos então concluir a seguinte Proposição:

Proposição 2.4. Um subconjunto $Z \subset \mathbb{P}_{k}^{n} \times \mathbb{P}_{k}^{m}$ é fechado se e só se $Z=$ $\mathcal{Z}\left(G_{1}, \ldots, G_{l}\right)$, com $G_{s} \in k\left[x_{0}, \ldots, x_{n}, y_{0}, \ldots, y_{m}\right]$ bi-homogêneo para todo $s=$ $1, \ldots, l$ e de bi-grau $\left(d_{s}, d_{s}\right)$. 
Porém, veremos que não precisamos pedir que o bi-grau tenha coordenadas iguais. De fato, seja $G \in k\left[x_{0}, \ldots, x_{n}, y_{0}, \ldots, y_{m}\right]$ um polinômio bihomogêneo de bi-grau $\left(d_{1}, d_{2}\right)$ e suponha sem perdas $d_{1} \leq d_{2}$. Então

$$
\mathcal{Z}(G)=\mathcal{Z}\left(x_{0}^{d_{2}-d_{1}} G, \ldots, x_{n}^{d_{2}-d_{1}} G\right)
$$

Isso significa que o conjunto de zeros $\mathcal{Z}(G)$ é também o conjunto de zeros de um conjunto finito de polinômios bi-homogêneos de bi-grau $\left(d_{2}, d_{2}\right)$. Assim, podemos descrever os fechados de $\mathbb{P}_{k}^{n} \times \mathbb{P}_{k}^{m}$ de forma menos rígida, conforme a seguinte Proposição:

Proposição 2.5. Um subconjunto $Z \subset \mathbb{P}_{k}^{n} \times \mathbb{P}_{k}^{m}$ é fechado se e só se $Z=$ $\mathcal{Z}\left(G_{1}, \ldots, G_{l}\right)$, com $G_{s} \in k\left[x_{0}, \ldots, x_{n}, y_{0}, \ldots, y_{m}\right]$ bi-homogêneo para todo $s=$ $1, \ldots, l$.

Identificando $\mathbb{A}_{k}^{n} \operatorname{com} \mathcal{U}_{0}=\mathbb{P}_{k}^{n} \backslash \mathcal{Z}\left(x_{0}\right)$, podemos entender $\mathbb{A}_{k}^{n} \times \mathbb{P}_{k}^{m}$ como $\mathcal{U}_{0} \times \mathbb{P}_{k}^{m}$, que é um aberto de $\mathbb{P}_{k}^{n} \times \mathbb{P}_{k}^{m}$, complementar ao fechado

$$
\mathcal{Z}\left(x_{0}\right)=\mathcal{Z}\left(x_{0} y_{0}, x_{0} y_{1}, \ldots, x_{0} y_{m}\right) \subset \mathbb{P}_{k}^{n} \times \mathbb{P}_{k}^{m}
$$

Portanto, $\mathbb{A}_{k}^{n} \times \mathbb{P}_{k}^{m}$ também pode ser visto como uma variedade quasi-projetiva, e não é difícil ver que os fechados de $\mathbb{A}_{k}^{n} \times \mathbb{P}_{k}^{m}$ são da forma $\mathcal{Z}\left(G_{1}, \ldots, G_{l}\right)$, com $G_{s} \in k\left[x_{0}, \ldots, x_{n}, y_{0}, \ldots, y_{m}\right]$ homogêneo nas variáveis $y_{i}$ para todo $s=1, \ldots, l$. 


\section{3}

\section{Morfismos e aplicações racionais}

Este Capítulo se propõe a esclarecer o que são as aplicações birracionais de $\mathbb{P}_{k}^{n}$ em $\mathbb{P}_{k}^{n}$ (os elementos do Grupo de Cremona) e também legitimizar sua composição (a operação de grupo). Para isso, é preciso antes entender o que são morfismos e aplicações racionais.

A partir de agora vamos omitir o corpo $k$ da notação $\mathbb{P}_{k}^{n}$ por $\mathbb{P}^{n}$. Para as seguintes definições, considere $X \subset \mathbb{P}^{n}$ e $Y \subset \mathbb{P}^{m}$ variedades projetivas e $A \subset X, B \subset Y$ variedades quasi-projetivas. Para referência do Capítulo consultar (SHAFAREVICH, 1994) c. 4.2 e 4.3 ou (HARTSHORNE, 1977) c. 1.3 e 1.4 .

\section{1}

\section{Morfismos e funções regulares}

Nesta Seção, nosso objetivo será estudar morfismos entre variedades quasi-projetivas. A seguinte Proposição de Topologia será usada como Lema para outras Proposições.

Proposição 3.1. Seja $T$ um espaço topológico e $\bigcup_{\alpha \in \Lambda} U_{\alpha}=T$ uma cobertura de $T$ por abertos. Se $F \subset T$ é tal que $F \cap U_{\alpha}$ é fechado em $U_{\alpha}$ para todo $\alpha \in \Lambda$, então $F$ é um fechado de $T$.

A partir de agora, por abuso de notação, quando $F_{i} \in k\left[x_{0}, x_{1}, \ldots, x_{n}\right]^{h}$, $i=0,1, \ldots, m$ forem polinômios homogêneos de mesmo grau em $n+1$ coordenadas e $Q=\left(q_{0}: q_{1}: \ldots: q_{n}\right) \in \mathbb{P}^{n}$, usaremos $\left(F_{0}(Q): F_{1}(Q): \ldots: F_{m}(Q)\right)$ para representar $\left(F_{0}\left(q_{0}, q_{1}, \ldots, q_{n}\right): F_{1}\left(q_{0}, q_{1}, \ldots, q_{n}\right): \ldots: F_{m}\left(q_{0}, q_{1}, \ldots, q_{n}\right)\right)$, e $\frac{F_{0}(Q)}{F_{1}(Q)}$ para representar $\frac{F_{0}\left(q_{0}, q_{1}, \ldots, q_{n}\right)}{F_{1}\left(q_{0}, q_{1}, \ldots, q_{n}\right)}$. Note que a hipótese de que os polinômios são homogêneos de mesmo grau faz com que as notações não dependam da escolha das coordenadas homogêneas de $Q$.

Definição 3.1. Um morfismo entre variedades quasi-projetivas $\mu: A \rightarrow B$ é uma aplicação localmente descrita por polinômios, ou seja, para todo $P \in A$ existe uma vizinhança aberta $U \subset A$ de $P$, e $m+1$ polinômios homogêneos de mesmo grau em $n+1$ coordenadas $F_{i} \in k\left[x_{0}, x_{1}, \ldots, x_{n}\right]^{h}, i=0,1, \ldots, m$ tais que para todo $Q \in U$, vale

$$
\mu(Q)=\left(F_{0}(Q): F_{1}(Q): \ldots: F_{m}(Q)\right)
$$


Um isomorfismo entre variedades quasi-projetivas é um morfismo $\mu: A \rightarrow$ $B$ bijetivo, cuja inversa também é um morfismo. Nesse caso dizemos que $A$ e $B$ são isomorfos.

Exemplo 11 (Projeções Lineares). Seja $E \subset \mathbb{P}^{n}$ subespaço linear de dimensão $m-1$, ou seja, $E=\mathcal{Z}\left(L_{0}, \ldots, L_{n-m}\right)$, onde os $L_{i}$ são polinômios homogêneos de grau 1 linearmente independentes. A projeção linear $\pi_{E}: \mathbb{P}^{n} \backslash E \rightarrow \mathbb{P}^{n-m}$ definida por $P \mapsto\left(L_{0}(P): \ldots: L_{n-m}(P)\right)$ é um morfismo. Note que $\pi_{E}$ está bem definido, pois as coordenadas serão todas nulas apenas se $P \in E$.

Podemos verificar que o fecho projetivo de uma fibra qualquer de $\pi_{E}$ é um subespaço linear de dimensão $m$ contendo $E$. De fato, seja $Q=\left(q_{0}: \ldots: q_{n-m}\right) \epsilon$ $\mathbb{P}^{n-m}$, então $\pi_{E}(P)=Q$ quando $\left(L_{0}(P): \ldots: L_{n-m}(P)\right)=\left(q_{0}: \ldots: q_{n-m}\right)$.

Como consequência,

$$
P \in Z=\mathcal{Z}\left(M_{i j} \mid\{i, j\} \subset\{0, \ldots, n-m\}\right),
$$

onde $M_{i j}=q_{i} L_{j}(P)-q_{j} L_{i}(P)$. E como o domínio de $\pi_{E}$ não inclui o fechado $E$, sabemos que $P \in Z \backslash E$. Não é difícil de ver que, na verdade, $\pi_{E}^{-1}(Q)=Z \backslash E$. Portanto $\pi_{E}^{-1}(Q)$ é um aberto não vazio de $Z, \operatorname{logo} \overline{\pi_{E}^{-1}(Q)}=Z$. Sendo assim, falta mostrar que $Z$ é um subespaço linear de dimensão $m$, pois é claro que contém $E$.

Note que $Z$ pode ser expresso com apenas $n-m$ polinômios homogêneos lineares. Se por exemplo $q_{0} \neq 0$, os polinômios $M_{0 j}$ são todos necessários para garantirmos

$$
P \in \mathcal{Z}\left(M_{0 j} \mid j \in\{1, \ldots, n-m\}\right) .
$$

Além disso, são linearmente independentes, já que $\sum_{j=1}^{n-m} a_{j} M_{0 j}=0$ implica $a_{j} q_{0}=0$ para todo $j=1, \ldots, n-m$, pois sabemos que os $L_{i}$ são linearmente independentes.

Exemplo 12. Na Seção 2.4, vimos que podemos identificar $\mathbb{P}^{n} \times \mathbb{P}^{m}$ com sua imagem pelo mergulho de Segre $\mathscr{S}\left(\mathbb{P}^{n} \times \mathbb{P}^{m}\right) \subset \mathbb{P}^{N}$, que por sua vez é uma variedade projetiva. Vamos ver que as projeções naturais

$$
\pi_{1} \circ \mathscr{S}^{-1}: \mathscr{S}\left(\mathbb{P}^{n} \times \mathbb{P}^{m}\right) \rightarrow \mathbb{P}^{n}, \pi_{2} \circ \mathscr{S}^{-1}: \mathscr{S}\left(\mathbb{P}^{n} \times \mathbb{P}^{m}\right) \rightarrow \mathbb{P}^{m}
$$

são morfismos.

Vimos que se $R=\left(r_{00}: r_{01}: \ldots: r_{n m}\right) \in \mathscr{S}\left(\mathbb{P}^{n} \times \mathbb{P}^{m}\right)$ e para $s, t$ fixos $r_{s t} \neq 0$, então $P=\left(r_{0 t}: r_{1 t}: \ldots: r_{n t}\right)$ e $Q=\left(r_{s 0}: r_{s 1}: \ldots: r_{s m}\right)$ são tais que $\mathscr{S}(P, Q)=R$. Isso significa que em

$$
\left(\mathcal{U}_{s t}=\mathbb{P}^{N} \backslash \mathcal{Z}\left(z_{s t}\right)\right) \cap \mathscr{S}\left(\mathbb{P}^{n} \times \mathbb{P}^{m}\right),
$$


o mapa $\pi_{1} \circ \mathscr{S}^{-1}$ pode ser descrito como $\left(r_{00}: r_{01}: \ldots: r_{n m}\right) \mapsto\left(r_{0 t}: r_{1 t}: \ldots: r_{n t}\right)$ e $\pi_{2} \circ \mathscr{S}^{-1}$ como $\left(r_{00}: r_{01}: \ldots: r_{n m}\right) \mapsto\left(r_{s 0}: r_{s 1}: \ldots: r_{s m}\right)$.

Como sabemos que a família $\left\{\mathcal{U}_{i j}\right\}_{i, j}$ é uma cobertura aberta de $\mathbb{P}^{N}$, podemos descrever $\pi_{1} \circ \mathscr{S}^{-1}$ e $\pi_{2} \circ \mathscr{S}^{-1}$ localmente em todo o domínio usando polinômios homogêneos de mesmo grau. Por definição, ambas as projeções são morfismos. E repare que não conseguimos descrevê-los globalmente com polinômios homogêneos de mesmo grau, mas sim em cada aberto $\mathcal{U}_{i j}$.

Também não é difícil ver que morfismos $\mu: \mathbb{P}^{n} \times \mathbb{P}^{m} \rightarrow \mathbb{P}^{l}$ são aplicações localmente definidas por polinômios bi-homogêneos de mesmo bigrau, ou seja, para todo $(P, Q) \in \mathbb{P}^{n} \times \mathbb{P}^{m}$ existe uma vizinhança aberta $U \subset \mathbb{P}^{n} \times \mathbb{P}^{m}$ de $(P, Q)$, e $l+1$ polinômios homogêneos de mesmo bigrau $F_{i} \subset k\left[x_{0}, \ldots, x_{n}, y_{0}, \ldots, y_{m}\right], i=0,1, \ldots, l$ tais que para todo $\left(P^{\prime}, Q^{\prime}\right) \in U$, vale

$$
\mu\left(P^{\prime}, Q^{\prime}\right)=\left(F_{0}\left(P^{\prime}, Q^{\prime}\right): F_{1}\left(P^{\prime}, Q^{\prime}\right): \ldots: F_{l}\left(P^{\prime}, Q^{\prime}\right)\right)
$$

Proposição 3.2. Morfismos são aplicações contínuas na topologia de Zariski.

Prova. Considere $\mu: A \rightarrow B$ um morfismo entre variedades quasi-projetivas $A \subset \mathbb{P}^{n}$ e $B \subset \mathbb{P}^{m}$. Seja $W \subset B$ um conjunto fechado em $B$. Nosso objetivo será mostrar que $Z=\mu^{-1}(W) \subset A$ é um conjunto fechado.

Seja $P \in A$ um ponto qualquer e considere $U \subset A$ vizinhança aberta de $P$ e $F_{0}, \ldots, F_{m} \in k\left[x_{0}, \ldots, x_{n}\right]$ polinômios homogêneos de mesmo grau tais que para todo $Q \in U$,

$$
\mu(Q)=\left(F_{0}(Q): F_{1}(Q): \ldots: F_{m}(Q)\right)
$$

Veremos que $Z \cap U$ é um fechado de $U$ e isso concluirá a prova devido à Proposição 3.1 .

De fato,

$$
Z \cap U=\left\{Q \in U \mid\left(F_{0}(Q): \ldots: F_{m}(Q)\right) \in W\right\}
$$

e observe que

$$
\left(F_{0}(Q): \ldots: F_{m}(Q)\right) \in W \Leftrightarrow G\left(F_{0}(Q), \ldots, F_{m}(Q)\right)=0 \forall G \in \mathcal{I}(W)
$$

Porém, para todo $G \in k\left[y_{0}, \ldots, y_{m}\right]^{h}$, temos que

$$
H_{G}(Q)=G\left(F_{0}(Q), \ldots, F_{m}(Q)\right)
$$

é um polinômio homogêneo nas coordenadas homogêneas de $Q$, ou seja, $H_{G} \in k\left[x_{0}, \ldots, x_{n}\right]^{h}$. 
Se definirmos

$$
S=\left\{H_{G}\right\}_{G \in \mathcal{I}(W)} \subset k\left[x_{0}, \ldots, x_{n}\right]^{h},
$$

podemos concluir que

$$
Z \cap U=\{Q \in U \mid H(Q)=0 \forall H \in S\}=\mathcal{Z}(S) \cap U
$$

é um fechado de $U$.

Também é possível definir um morfismo entre variedades quasi-projetivas usando funções regulares, como veremos em breve.

Definição 3.2. Seja $U \subset \mathbb{P}_{k}^{n}$ uma variedade quasi-projetiva e um ponto $P \in U$. Uma função $f: U \rightarrow k$ é dita regular em $P$ se existem $U_{P} \subset U$ aberto contendo $P$ e polinômios $F, G \in k\left[x_{0}, \ldots, x_{n}\right]^{h}$ homogêneos de mesmo grau tais que

$$
\forall Q \in U_{P}\left(G(Q) \neq 0 \wedge f(Q)=\frac{F(Q)}{G(Q)}\right) .
$$

Dizemos que $f$ é regular em $U$ quando é regular em todo ponto de $U$. A $k$ álgebra das funções regulares em $U$ é denotada por $\mathcal{O}(U)$.

Funções regulares $f: U \rightarrow k$ também podem ser vistas como morfismos $\widetilde{f}: U \rightarrow \mathcal{U}_{0}=\mathbb{P}_{k}^{1} \backslash \mathcal{Z}\left(x_{0}\right)$. De fato, o mapa $P \mapsto(1: f(P))$ satisfaz que para todo $P \in U$ existe uma vizinhança aberta de $P$ e polinômios $F, G \in k\left[x_{0}, \ldots, x_{n}\right]^{h}$ tais que $(1: f(Q))=(G(Q): F(Q))$ para todo $Q$ na vizinhança. É fácil ver que a correspondência $f \leftrightarrow \widetilde{f}$ é bijetiva.

Proposição 3.3. Seja $\mu: A \rightarrow B$ um morfismo, $V \subset B$ um aberto $e$ $U=\mu^{-1}(V) \subset A$. Se $f$ é uma função regular em $V$, então $f \circ \mu$ é uma função regular em $U$.

Prova. Sejam $P \in U$ qualquer e $Q=\mu(P) \in V$ sua imagem por $\mu$. Sabemos que existe uma vizinhança aberta de $P$, que chamaremos de $U_{P}$, onde $\mu$ é descrita pelos $m+1$ polinômios homogêneos de mesmo grau $F_{0}, \ldots, F_{m} \in k\left[x_{0}, \ldots, x_{n}\right]^{h}$.

Além disso, como $f \in \mathcal{O}(V)$, também sabemos que existe uma vizinhança aberta de $Q, V_{Q} \subset V$ e polinômios homogêneos de mesmo grau $F, G \in$ $k\left[x_{0}, \ldots, x_{n}\right]^{h}$ onde para todo $R \in V_{Q}$ vale $f(R)=\frac{F(R)}{G(R)}$. Podemos definir $U_{P}^{\prime}=\mu^{-1}\left(V_{Q}\right) \subset A$, e como $\mu$ é contínua, $U_{P}^{\prime}$ é uma vizinhança aberta de $P$.

Agora basta observar que a interseção $U_{P} \cap U_{P}^{\prime} \subset A$ é uma vizinhança aberta de $P$, onde $\mu$ é descrita pelos $m+1$ polinômios $F_{0}, \ldots, F_{m} \in k\left[x_{0}, \ldots, x_{n}\right]$ 
e $f$ pelos polinômios $F, G \in k\left[x_{0}, \ldots, x_{n}\right]^{h}$. Portanto, para todo $R$ nesse aberto,

$$
f \circ \mu(R)=\frac{F\left(F_{0}(R): \ldots: F_{m}(R)\right)}{G\left(F_{0}(R): \ldots: F_{m}(R)\right)} .
$$

Como $F\left(F_{0}(R): \ldots: F_{m}(R)\right)$ e $G\left(F_{0}(R): \ldots: F_{m}(R)\right)$ são polinômios homogêneos de mesmo grau nas coordenadas homogêneas de $R$, e $G \circ \mu$ não se anula em $U_{P}^{\prime}$, isso mostra que $f \circ \mu$ é regular em $P$. Por fim, $f \circ \mu$ é regular em $U$.

Proposição 3.4. Seja $\mu: A \rightarrow B$ uma aplicação contínua. Suponha que para todo aberto $V \subset B$, com $U=\mu^{-1}(V)$, e toda função $f \in \mathcal{O}(V)$ regular em $V$, tem-se que $f \circ \mu \in \mathcal{O}(U)$. Então $\mu$ é um morfismo.

Prova. Começaremos fixando um $P=\left(p_{0}: p_{1}: \ldots: p_{n}\right) \in A$ qualquer e $Q=\mu(P)=$ $\left(q_{0}: q_{1}: \ldots: q_{m}\right) \in B$. Suponha sem perdas que $q_{0} \neq 0$.

Seja $V=B \backslash \mathcal{Z}\left(y_{0}\right)$, considere $f_{i}: V \rightarrow k$ que mapeia $\left(b_{0}: \ldots: b_{m}\right) \mapsto \frac{b_{i}}{b_{0}}$, $i=1, \ldots, m$. Cada $f_{i}$ é regular em $V$, e portanto $\mu_{i}=f_{i} \circ \mu$ é regular em $U$, onde $U=\mu^{-1}(V)$.

Portanto para cada $i=1, \ldots, m$, existem vizinhanças abertas $U_{P}^{i} \subset U$ de $P$ e polinômios homogêneos de mesmo grau $F_{i}, G_{i} \in k\left[x_{0}, \ldots, x_{n}\right]^{h}$, tais que $\mu_{i}(R)=\frac{F_{i}(R)}{G_{i}(R)}$ para todo $R \in U_{P}^{i}$.

Em particular, na interseção $\bigcap_{i=1}^{m} U_{P}^{i}$, temos que

$$
\mu(R)=\left(1: \mu_{1}(R): \ldots: \mu_{m}(R)\right)=\left(1: \frac{F_{1}(R)}{G_{1}(R)}: \ldots: \frac{F_{m}(R)}{G_{m}(R)}\right) .
$$

Podemos multiplicar todas as coordenadas homogêneas pelo produtório $\prod_{i=1}^{m} G_{i}$ e então teremos que $\mu$ é descrita por polinômios homogêneos de grau $\sum_{i=1}^{m} \operatorname{deg}\left(G_{i}\right)$. Portanto $\mu$ é um morfismo.

As Proposições anteriores nos permitem usar a seguinte definição alternativa para morfismos entre variedades quasi-projetivas.

Definição 3.3. Um morfismo entre variedades quasi-projetivas $\mu: A \rightarrow B$ é uma aplicação com as seguintes propriedades:

- $\mu$ é contínua na topologia de Zariski;

- Para todo aberto $V \subset B$, com $U=\mu^{-1}(V)$, e toda função $f \in \mathcal{O}(V)$ regular em $V$, tem-se que $f \circ \mu \in \mathcal{O}(U)$ é uma função regular em $U$.

Proposição 3.5. Sejam $\mu: A \rightarrow B$ e $\nu: B \rightarrow C$ morfismos entre variedades quasi-projetivas, então o mapa $\nu \circ \mu: A \rightarrow C$ é um morfismo entre variedades quasi-projetivas. 
Proposição 3.6. Sejam $U$ uma variedade quasi-projetiva, $f, g: U \rightarrow k$ duas funções regulares em $U$ e $U^{\prime} \subset U$ um aberto não vazio. Se $f(P)=g(P)$ para todo $P$ em $U^{\prime}$, então $f=g$.

Prova. Podemos supor que $g$ é identicamente nula, caso contrário bastaria trocar $f$ por $f-g$. Vamos mostrar que $Z=\{P \in U \mid f(P)=0\}$ é um fechado de $U$, que implica $U=\overline{U^{\prime}} \subset Z$ e então $U=Z$.

Sabemos que para todo $P \in U$, existem $U_{P} \subset U$, e polinômios $F, G$ como na definição 3.2. Portanto

$$
Z \cap U_{P}=\mathcal{Z}\left(F_{P}\right) \cap U_{P}
$$

é um fechado de $U_{P}$. Como $U=\cup U_{P}, Z$ é um fechado de $U$, pela Proposição 3.1 .

Proposição 3.7. Sejam $\mu, \nu: A \rightarrow B$ dois morfismos e $U \subset A$ um aberto não vazio. Se $\mu(P)=\nu(P)$ para todo $P$ em $U$, então $\mu=\nu$.

Prova. Segue da prova da Proposição 3.4

Dado um morfismo $\mu: A \rightarrow B$ entre variedades quasi-projetivas, podemos definir um homomorfismo de $k$-álgebras $\mu^{*}: \mathcal{O}(B) \rightarrow \mathcal{O}(A)$ que mapeia $f \mapsto f \circ \mu$, conhecido como o pullback de $\mu$.

A correspondência $\mu \mapsto \mu^{*}$ é funtorial, isto é, se Id $: A \rightarrow A$ é o morfismo identidade em $A$, então $\operatorname{Id}^{*}: \mathcal{O}(A) \rightarrow \mathcal{O}(A)$ é a identidade de $\mathcal{O}(A)$, e se $\mu: A \rightarrow B$ e $\nu: B \rightarrow C$ são dois morfismos, então $(\nu \circ \mu)^{*}=\mu^{*} \circ \nu^{*}$.

\section{2 \\ Aplicações racionais}

Já vimos que nem sempre um morfismo $\mu: A \rightarrow B$ entre variedades quasiprojetivas, com $B \subset \mathbb{P}^{m}$ pode ser descrito globalmente por $m+1$ polinômios homogêneos de mesmo grau. Então precisamos de uma cobertura por abertos de $A$ de forma que $\mu$ possa ser descrita por $m+1$ polinômios homogêneos de mesmo grau em cada um dos abertos.

Agora suponha que começamos com um aberto não vazio $U \subset A$ e que temos $m+1$ polinômios homogêneos de mesmo grau $F_{i}$, com $i=0, \ldots, m$, de forma que a aplicação $Q \mapsto\left(F_{0}(Q): \ldots: F_{m}(Q)\right)$ está bem definida em $U$. Sabemos pela Proposição 3.7 que existe no máximo um morfismo $\mu: A \rightarrow \mathbb{P}^{m}$ de forma que a aplicação acima seja exatamente $\left.\mu\right|_{U}$. Porém, muitas vezes não existe um morfismo assim definido em $A$.

Apesar disso, uma aplicação mesmo que definida em um aberto não vazio de $A$ já carrega muita informação, já que abertos não vazios de variedades 
quasi-projetivas são densos. Uma aplicação assim é uma aplicação racional de $A$ em $\mathbb{P}^{m}$, e sua definição vem a seguir.

Definição 3.4. Uma aplicação racional entre variedades quasi-projetivas $\varphi: A \rightarrow B$ é uma classe de equivalência de pares $\left(U, \varphi_{U}\right)$, onde $U \subset A$ é um aberto não vazio e $\varphi_{U}: U \rightarrow B$ é um morfismo, com a relação de equivalência $\left(U, \varphi_{U}\right) \sim\left(V, \varphi_{V}\right)$ quando $\left.\varphi_{U}\right|_{U \cap V}=\left.\varphi_{V}\right|_{U \cap V}$.

Repare que essa relação de equivalência é bem definida devido à Proposição 3.7, e que a definição acima é uma generalização da definição de morfismo, ou seja, morfismos $\mu: A \rightarrow B$ são sempre aplicações racionais de $A$ em $B$.

Exemplo 13. Sejam $F_{i}, i=0,1,2$ polinômios homogêneos de mesmo grau em 3 variáveis não todos nulos. Considere uma aplicação $\varphi: \mathbb{P}^{2} \rightarrow \mathbb{P}^{2}$ que leva cada ponto $P \in \mathbb{P}^{2}$ em $\left(F_{0}(P): F_{1}(P): F_{2}(P)\right)$. Essa aplicação nem sempre está definida em todo ponto, pois pode ser que $F_{0}(P)=F_{1}(P)=F_{2}(P)=0$ para $\operatorname{algum} P$.

Seja $Z=\mathcal{Z}\left(F_{0}, F_{1}, F_{2}\right) \subset \mathbb{P}^{2}$ o conjunto de tais pontos. Se $Z=\varnothing$, então $\varphi$ é um morfismo, mas, no caso geral, apenas sua restrição $\left.\varphi\right|_{\mathbb{P}^{2} \backslash Z}$ é um morfismo. Como $Z$ é um fechado e os $F_{i}$ 's não são todos nulos, $\mathbb{P}^{2} \backslash Z$ é um aberto não vazio, e dizemos que $\varphi$ é uma aplicação racional, que corresponde à classe de $\left(\mathbb{P}^{2} \backslash Z,\left.\varphi\right|_{\mathbb{P}^{2} \backslash Z}\right)$.

Exemplo 14 . Considere a aplicação racional $\varphi: \mathbb{P}^{2} \rightarrow \mathbb{P}^{2}$ definida pelos polinômios $F_{0}=x_{0}^{2}, F_{1}=x_{0} x_{1}$ e $F_{2}=x_{0} x_{2}$. Apesar de essa forma de representar $\varphi$ não estar definida nos pontos onde $x_{0}=0, \varphi$ se comporta como o morfismo identidade nos outros pontos, e por isso $\varphi$ e a identidade são equivalentes como aplicações racionais. Note que sempre podemos pedir que os polinômios que descrevem uma aplicação racional não tenham fator comum.

Exemplo 15. Voltando ao exemplo da projeção linear, seja $E=$ $\mathcal{Z}\left(L_{0}, \ldots, L_{n-m}\right) \subset \mathbb{P}^{n}$, onde os $L_{i}$ são polinômios homogêneos de grau 1 linearmente independentes, e o morfismo $\pi_{E}: \mathbb{P}^{n} \backslash E \rightarrow \mathbb{P}^{n-m}$ tal que $P \mapsto\left(L_{0}(P): \ldots: L_{n-m}(P)\right)$. Seja $\varphi_{E}: \mathbb{P}^{n} \rightarrow \mathbb{P}^{n-m}$ a aplicação racional classe de $\left(\mathbb{P}^{n} \backslash E, \pi_{E}\right)$.

Note que $\varphi_{E}$ não é um morfismo. Se fosse, então teríamos que cada fibra de $\varphi_{E}$ seria um fechado de $\mathbb{P}^{n}$, afinal morfismos são aplicações contínuas. Mas pelo que já estudamos desse exemplo, o fecho de cada fibra de $\pi_{E}$ contém $E$. Juntando essas duas informações, temos que cada fibra de $\varphi_{E}$ contém $E$, um absurdo.

Exemplo 16. A Transformação Quadrática Padrão, $\Phi: \mathbb{P}^{2} \quad \rightarrow \quad \mathbb{P}^{2}$, é a classe do morfismo $(x: y: z) \mapsto(y z: x z: x y)$, definido no aberto 
$\mathbb{P}^{2} \backslash\{(0: 0: 1),(0: 1: 0),(1: 0: 0)\}$. Se $L_{12}=\mathcal{Z}(x), L_{02}=\mathcal{Z}(y), L_{01}=\mathcal{Z}(z)$ são as retas em $\mathbb{P}^{2}$ onde $x=0, y=0, z=0$ respectivamente, é fácil ver que $\Phi$ as contrai respectivamente nos pontos $P_{0}=(1: 0: 0), P_{1}=(0: 1: 0), P_{2}=(0: 0: 1)$.

Além disso, no aberto $A=\mathbb{P}^{2} \backslash\left(L_{12} \cup L_{02} \cup L_{01}\right)$, podemos escrever $\Phi:(x: y: z) \mapsto(y z: x z: x y)=\left(\frac{1}{x}: \frac{1}{y}: \frac{1}{z}\right) \in A$. Portanto, $\left.\left.\Phi\right|_{A} \circ \Phi\right|_{A}$ é a aplicação identidade, e então $\left.\Phi\right|_{A}$ é um automorfismo de $A$.

Em geral, não podemos compor duas aplicações racionais. Por exemplo, sejam $\varphi: \mathbb{P}^{2} \rightarrow \mathbb{P}^{2}$ tal que $(x: y: z) \mapsto(1: 0: 0)$ e $\psi: \mathbb{P}^{2} \rightarrow \mathbb{P}^{1}$ tal que $(x: y: z) \mapsto(y: z)$. A composição $\psi \circ \varphi$ não estaria definida em nenhum ponto, enquanto que uma aplicação racional precisa estar definida em um aberto não vazio. Para conseguirmos compor aplicações racionais, pedimos que sua imagem seja um aberto não vazio, propriedade que definiremos a seguir como "dominante".

Definição 3.5. Dizemos que o domínio de definição de uma aplicação racional $\varphi: A \rightarrow B$ é o maior aberto $U \subset A$ dentre os representantes $\left(U, \varphi_{U}\right)$, e seu complementar em $A$ é dito o conjunto de indeterminação de $\varphi$. Um ponto em seu conjunto de indeterminação é dito um polo ou ponto de indeterminação. Dizemos que $\varphi$ é dominante quando o subconjunto $\varphi_{U}(U) \subset B$ é denso, para algum (e portanto para todo) representante $\left(U, \varphi_{U}\right)$.

Se $\varphi: A \rightarrow B$ e $\psi: B \rightarrow C$ são duas aplicações racionais dominantes, podemos definir a aplicação racional dominante $\psi \circ \varphi$ como a seguir.

Sejam $U$ e $V$ os respectivos domínios de definição de $\varphi$ e $\psi$ e considere $\widetilde{U}=\varphi_{U}^{-1}(V)$. Este último é um aberto de $U$ (pois $\varphi_{U}$ é contínua) e portanto aberto de $A$. Também é não vazio, caso contrário teríamos $\varphi_{U}(U) \subset B \backslash V$, e como $B \backslash V$ é um fechado de $B, \overline{\varphi_{U}(U)} \subset B \backslash V \neq B$, um absurdo, pois $\varphi$ é dominante.

Agora que sabemos que $\widetilde{U}$ é um aberto não vazio de $A$, considere o morfismo $\mu=\left.\psi_{V} \circ \varphi_{U}\right|_{\widetilde{U}}: \widetilde{U} \rightarrow B$. Ele define uma aplicação racional entre $A$ e $C$. Para mostrar que tal aplicação racional é dominante, basta mostrar que $\mu(\widetilde{U})$ é denso em $C$.

Seja $\widetilde{V}=\varphi_{U}(\widetilde{U})$, queremos mostrar que $\mu(\widetilde{U})=\psi_{V}(\widetilde{V})$ é denso em $C$. Veja que também podemos expressar $\widetilde{V}=V \cap \varphi_{U}(U)$, e desta forma, como $\varphi$ é dominante, $\varphi_{U}(U)$ é denso em $B$ e portanto $\widetilde{V}$ é denso em $V$.

Se $\psi_{V}(\widetilde{V})$ não fosse denso em $C$, existiria um fechado próprio $Z \subset C$ tal que $\psi_{V}(\widetilde{V}) \subset Z$, o que implicaria em $\widetilde{V} \subset \psi_{V}^{-1}(Z)$. Por sua vez, $\psi_{V}^{-1}(Z)$ é um fechado de $V$, pois $\psi_{V}$ é contínua, e próprio, caso contrário $\psi_{V}(V) \subset Z$ não seria denso em $C$. Isso significa que $\widetilde{V}$ não é denso em $V$, o que é uma contradição. 
Agora que sabemos compor aplicações racionais dominantes, podemos definir aplicações birracionais.

Definição 3.6. Uma aplicação racional dominante $\varphi: A \rightarrow B$ é dita birracional quando existe uma aplicação racional dominante $\psi: B \rightarrow A$ tal que $\psi \circ \varphi$ é a aplicação identidade em $A$ e $\varphi \circ \psi$ é a aplicação identidade em $B$.

Nesse caso, dizemos que $A$ e $B$ são birracionalmente equivalentes. Como já vimos que podemos compor aplicações racionais dominantes, esta é uma relação de equivalência.

Quando $A=B=\mathbb{P}^{2}$, dizemos que $\varphi$ e $\psi$ são Transformações de Cremona.

Exemplo 17. A Transformação Quadrática Padrão $\Phi: \mathbb{P}^{2} \rightarrow \mathbb{P}^{2}$ definida por $(x: y: z) \mapsto(y z: x z: x y)$ vista anteriormente é uma Transformação de Cremona. De fato, já vimos que $\left.\Phi\right|_{A}$ com $A=\mathbb{P}^{2} \backslash\left\{L_{12} \cup L_{02} \cup L_{01}\right\}$ é um automorfismo. Então, como aplicação racional em $\mathbb{P}^{2}, \Phi \circ \Phi$ é equivalente a $\left.\left.\Phi\right|_{A} \circ \Phi\right|_{A}=\operatorname{Id}_{A}$, que por sua vez é equivalente a $\operatorname{Id}_{\mathbb{P}^{2}}$.

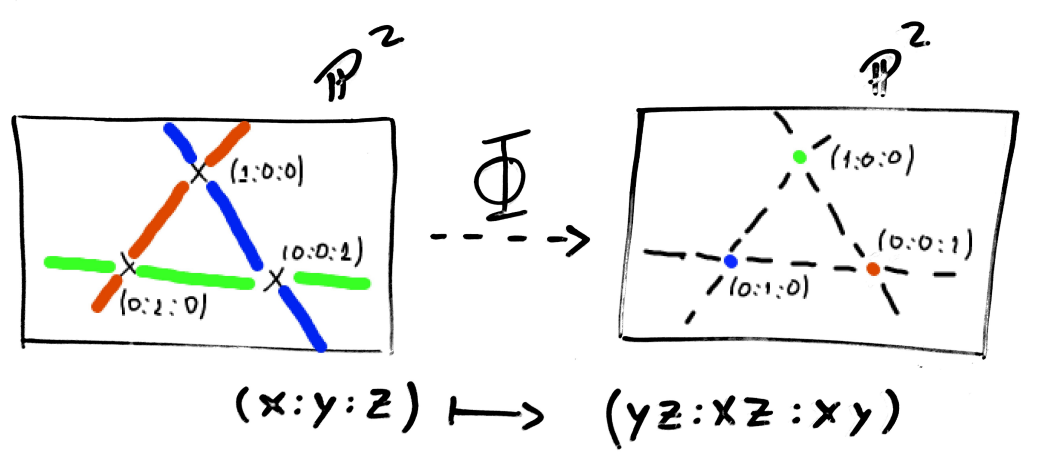

Definição 3.7. O Grupo de Cremona é o conjunto das Transformações de Cremona

$$
C r\left(\mathbb{P}^{2}\right)=\left\{\varphi: \mathbb{P}^{2} \rightarrow \mathbb{P}^{2} \mid \varphi \text { é aplicação birracional }\right\}
$$

munido da operação de composição.

Como morfismos são em particular aplicações racionais, automorfismos de $\mathbb{P}^{2}$ são também aplicações birracionais de $\mathbb{P}^{2}$ nele mesmo, portanto pertencem ao Grupo de Cremona. Em outras palavras, $\operatorname{Aut}\left(\mathbb{P}^{2}\right)$ é um subgrupo de $\operatorname{Cr}\left(\mathbb{P}^{2}\right)$. 
Por sua vez a Transformação Quadrática Padrão $\Phi$ (que não é um morfismo) também pertence a $\operatorname{Cr}\left(\mathbb{P}^{2}\right)$. O Teorema de Nöether-Castelnuovo diz respeito ao subgrupo

$$
\left\langle\operatorname{Aut}\left(\mathbb{P}^{2}\right), \Phi\right\rangle \subset \operatorname{Cr}\left(\mathbb{P}^{2}\right)
$$

das transformaçoes birracionais de $\mathbb{P}^{2}$ nele mesmo gerado pelos automorfismos de $\mathbb{P}^{2}$ e $\Phi$. Segundo o Teorema, a inclusão é, na verdade, uma igualdade.

Teorema 3.1 (Nöether-Castelnuovo (CASTELNUOVO, 1901)). O Grupo de Cremona é gerado por automorfismos de $\mathbb{P}^{2}$ e $\Phi$, isto é,

$$
\operatorname{Cr}\left(\mathbb{P}^{2}\right)=\left\langle A u t\left(\mathbb{P}^{2}\right), \Phi\right\rangle
$$

\section{3}

\section{Funções racionais e dimensão}

Assim como as funções regulares para os morfismos, as funções racionais têm um papel muito importante para as aplicações racionais. Além disso, nos ajudarão a definir a dimensão de uma variedade de forma algébrica.

Definição 3.8. Uma função racional $f$ em A é uma classe de equivalência de pares $\left(U, f_{U}\right)$, onde $U \subset A$ é um aberto não vazio e $f_{U} \in \mathcal{O}(U)$ é uma função regular em $U$, e dizemos que $\left(U, f_{U}\right) \sim\left(V, f_{V}\right)$ quando $\left.f_{U}\right|_{U \cap V}=\left.f_{V}\right|_{U \cap V}$. O corpo das funções racionais em $A$ é o conjunto das funções racionais, denotado por $K(A)$.

A relação acima é uma relação de equivalência, graças à Proposição 3.6. Toda função regular $f: A \rightarrow k$ é em particular uma função racional. Em outras palavras, $\mathcal{O}(A) \subset K(A)$.

Por definição, se $U \subset A$ é um aberto não vazio, então $K(U) \cong_{k} K(A)$.

Definição 3.9. A dimensão de uma variedade quasi-projetiva $X$ é o grau de transcendência da extensão de corpos $K(X) \mid k$.

Com isso, fica fácil observar que dado um aberto $U$ de uma variedade quasi-projetiva $A, K(U)=K(A)$ implica que $\operatorname{dim}(U)=\operatorname{dim}(A)$

Exemplo 18. Quando $A$ é uma variedade afim, $K(A)$ pode ser identificado com o corpo quociente de $\mathcal{A}(A)$. Em particular, $K\left(\mathbb{A}^{n}\right) \cong_{k} k\left(x_{1}, x_{2}, \ldots x_{n}\right)$. Coerentemente, temos $\operatorname{dim} \mathbb{A}^{n}=n$. Como $\mathbb{A}^{n}$ é um aberto de $\mathbb{P}^{n}$, é claro que $\operatorname{dim}\left(\mathbb{P}^{n}\right)=n$.

Também vale a seguinte propriedade, provada em (SHAFAREVICH, 1994) 6.2 Teorema 5: 
Proposição 3.8. Seja $X \subset \mathbb{P}^{n}$ uma variedade projetiva e $F \in k\left[x_{0}, \ldots, x_{n}\right]^{h}$ um polinômio homogêneo tal que $F \notin \mathcal{I}(X)$. Então toda componente irredutivel de $X \cap \mathcal{Z}(F)$ tem dimensão igual a $\operatorname{dim}(X)-1$.

Agora vamos introduzir a noção de pullback de funções racionais, que será muito importante quando tratarmos de divisores. Mais propriedades sobre dimensão se encontram em (SHAFAREVICH, 1994) c. 1.6 e (HARTSHORNE, 1977) c. 1.

Definição 3.10. Dada uma aplicação racional dominante $\varphi: A \rightarrow B$ entre variedades quasi-projetivas, podemos definir o pullback de $\varphi$ como o monomorfismo de corpos $\varphi^{*}: K(B) \rightarrow K(A)$ pefinida por $f \mapsto f \circ \varphi$. A correspondência $\varphi \mapsto \varphi^{*}$ é funtorial e contravariante.

Proposição 3.9. Duas variedades quasi-projetivas $A$ e B são birracionalmente equivalentes se e só se $K(A) \cong_{k} K(B)$. Em particular, se $A$ e $B$ são birracionalmente equivalentes, então $\operatorname{dim}(A)=\operatorname{dim}(B)$.

Como referência, consultar (HARTSHORNE, 1977) Corolário 4.5

Exemplo 19. Já vimos que o espaço afim $\mathbb{A}^{n}$ pode ser identificado com $\mathcal{U}_{0}=\mathbb{P}^{n} \backslash \mathcal{Z}\left(x_{0}\right)$. Por sua vez, a variedade quase projetiva $\mathcal{U}_{0}$ é birracionalmente equivalente a $\mathbb{P}^{n}$ pelo mapa de inclusão, portanto

$$
K\left(\mathbb{P}^{n}\right) \cong_{k} K\left(\mathbb{A}^{n}\right) \cong_{k} k\left(x_{1}, x_{2}, \ldots, x_{n}\right) .
$$

Relembre que estamos especialmente interessados no grupo $\operatorname{Cr}\left(\mathbb{P}^{2}\right)$ das Transformações de Cremona $\varphi: \mathbb{P}^{2} \rightarrow \mathbb{P}^{2}$. Sendo assim, o monomorfismo de $k$-álgebras $\varphi^{*}: K\left(\mathbb{P}^{n}\right) \rightarrow K\left(\mathbb{P}^{n}\right)$ possui uma inversa $\left(\varphi^{*}\right)^{-1}=\left(\varphi^{-1}\right)^{*}$. Portanto $\varphi^{*}$ é um $k$-automorfismo de $K\left(\mathbb{P}^{n}\right) \cong_{k} k\left(x_{1}, x_{2}, \ldots, x_{n}\right)$, ou seja,

$$
\varphi^{*} \in \operatorname{Aut}_{k}\left(k\left(x_{1}, x_{2}, \ldots, x_{n}\right)\right) .
$$

Mais ainda, a correspondência funtorial $\operatorname{Cr}\left(\mathbb{P}^{2}\right) \rightarrow \operatorname{Aut}_{k}\left(K\left(\mathbb{P}^{n}\right)\right.$ dada por $\varphi \mapsto \varphi^{*}$ é bijetiva. De fato, não é difícil construir uma Transformação de Cremona $\varphi$ dado um $k$-automorfismo $\psi \in K\left(\mathbb{P}^{n}\right)$ tal que $\varphi^{*}=\psi$. Em outras palavras, o grupo $\mathrm{Cr}\left(\mathbb{P}^{2}\right)$ pode ser identificado com

$$
\operatorname{Cr}\left(\mathbb{P}^{2}\right) \cong \operatorname{Aut}_{k}\left(k\left(x_{1}, x_{2}, \ldots, x_{n}\right)\right) .
$$

A partir de agora, estaremos especialmente interessados em superfícies não-singulares. Como superfície, entendemos variedades quasi-projetivas de dimensão 2. Já a noção de singularidade pode ser encontrada em (SHAFARE- 
VICH, 1994) Capítulo 2.1 e (HARTSHORNE, 1977) Capítulo 1.5. Brevemente, considere a segunte definição:

Definição 3.11. Dizemos que uma variedade quasi-projetiva $X$ é não-singular no ponto $P \in X$ se o anel local de $X$ em $P$

$$
\mathcal{O}_{P}(X)=\{f \in K(X) \mid f \text { é regular em } P\}
$$

é regular, isto é, se

$$
\operatorname{dim}_{k}\left(m / m^{2}\right)=\operatorname{dim}_{\text {Krull }}\left(\mathcal{O}_{P}(X)\right)
$$

onde $m$ é o ideal maximal de $\mathcal{O}_{P}(X)$ dado por

$$
m=\left\{f \in \mathcal{O}_{P}(X) \mid f(P)=0\right\}
$$

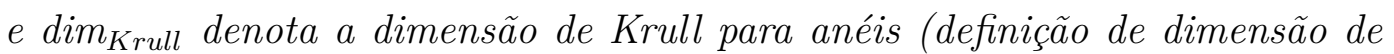
Krull em (KLEIMAN; ALTMAN, 2018) Parte I Seção 15).

Dizemos que $X$ é não-singular se for não-singular em todo ponto $P \in X$. 


\section{4}

\section{Blowup de superfícies}

O blowup de variedades algébricas é uma construção fundamental em Geometria Algébrica. Além de ser o exemplo mais simples de morfismo birracional que não é um isomorfismo, o blowup serve como ferramenta para entendermos melhor aplicações racionais e birracionais. Como referência para este Capítulo, considere (SHAFAREVICH, 1994) Parte 4 Capítulos 3.1, 3.3 e 3.4

Seja $S$ uma superfície quasi-projetiva não-singular e $P \in S$ um ponto. A grosso modo, o blowup substitui $P$ pelo $\mathbb{P}^{1}$ de direções tangentes. Mais precisamente, um blowup de $S$ em $P$ é um par $\left(S_{P}, \pi\right)$, onde $S_{P}$ é uma superfície quasi-projetiva não-sigular, e $\pi: S_{P} \rightarrow S$ um morfismo com as seguintes propriedades:

- o chamado divisor excepcional $E=\pi^{-1}(P)$ é isomorfo a $\mathbb{P}^{1}$;

- o morfismo $\left.\pi\right|_{S_{P} \backslash E}$ é um isomorfismo entre $S_{P} \backslash E$ e $S \backslash\{P\}$.

Em particular, $\pi$ é um morfismo e também uma aplicação birracional, por isso é dito um morfismo birracional. O seguinte Teorema mostra que em algum sentido um blowup é o mais simples morfismo birracional que não é isomorfismo.

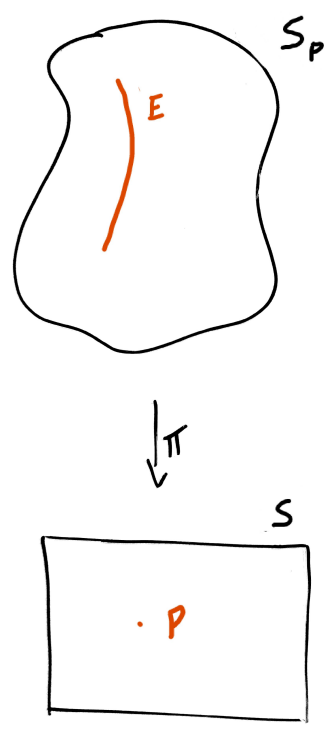

Teorema 4.1. Seja $\pi: X \rightarrow Y$ um morfismo birracional entre superfícies projetivas não-singulares que não é um isomorfismo. Então $\pi$ é a composição de finitos blowups. 
Este Teorema está na referência do Capítulo como Teorema 4.10

Dados $S$ e $P$ como acima, sempre existe um par $\left(S_{P}, \pi\right)$ satisfazendo as duas condições. Além disso, se $\left(S_{P}, \pi\right)$ e $\left(S_{P}^{\prime}, \pi^{\prime}\right)$ são blowups de $S$ no ponto $P \in S$, então existe um isomorfismo $\mu: S_{P} \rightarrow S_{P}^{\prime}$ tal que $\pi^{\prime} \circ \mu=\pi$. Neste sentido, dizemos que o blowup de uma superfície quasi-projetiva não-singular em qualquer ponto existe e é único. A prova destas afirmações será omitida, mas pode ser encontrada na referência do Capítulo.

Dizemos que a transformada estrita de uma curva $C \subset S$ pelo blowup de $S$ em $P$ é

$$
\widetilde{C}=\overline{\pi^{-1}(C \backslash\{P\})} \subset S_{P}
$$
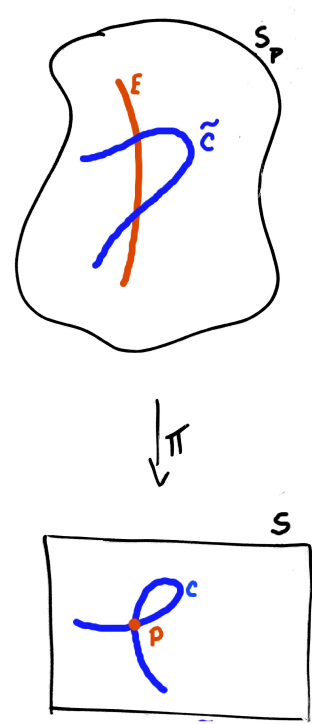

4.1

\section{0 blowup de $\mathbb{P}^{2}$ em um ponto}

Seja $P=(1: 0: 0)$, é fácil representar $\mathbb{P}_{P}^{2}$ como uma variedade projetiva em $\mathbb{P}^{2} \times \mathbb{P}^{1}$, basta tomar

$$
X=\left\{(x: y: z ; s: t) \in \mathbb{P}^{2} \times \mathbb{P}^{1} \mid y t-z s=0\right\}
$$

e $\pi: X \rightarrow \mathbb{P}^{2}$ a projeção natural de $X$ em $\mathbb{P}^{2}$.

De fato,

$$
\mathcal{E}=\pi^{-1}(P)=\{(1: 0: 0 ; s: t)\} \cong \mathbb{P}^{1},
$$

enquanto que para todo ponto $Q=\left(q_{0}: q_{1}: q_{2}\right) \in \mathbb{P}^{2} \backslash\{P\}$,

$$
\pi^{-1}(Q)=\left\{\left(q_{0}: q_{1}: q_{2} ; s: t\right) \mid q_{1} t=q_{2} s\right\} .
$$


Como $Q \neq P$, ou $q_{1}$ ou $q_{2}$ é não nulo. Se, sem perdas, $q_{1} \neq 0$, então $t=\frac{q_{2}}{q_{1}} s$, logo $(s: t)=\left(1: \frac{q_{2}}{q_{1}}\right)=\left(q_{1}: q_{2}\right) \mathrm{e}$

$$
\pi^{-1}(Q)=\left\{\left(q_{0}: q_{1}: q_{2} ; q_{1}: q_{2}\right)\right\}
$$

é um único ponto, e o mapa $Q \mapsto \pi^{-1}(Q)$ é um morfismo de $\mathbb{P}^{2} \backslash\{P\}$ para $X \backslash \mathcal{E}$. Com isso, podemos confirmar que $X$ é de fato o blowup de $\mathbb{P}^{2}$ em $P$, mas ainda podemos entendê-lo melhor.

Podemos cobrir $X$ com os abertos $X_{x}=X \backslash \mathcal{Z}(x), X_{y}=X \backslash \mathcal{Z}(y)$ e $X_{z}=X \backslash \mathcal{Z}(z)$. Destes, os dois últimos não contém o ponto $P$, e portanto $\pi$ restrito a cada um deles é um isomorfismo com um aberto de $\mathbb{P}^{2}$, o qual podemos identificar com $\mathbb{A}^{2}$. Já o aberto

$$
X_{x} \cong\left\{(y, z ; s: t) \in \mathbb{A}^{2} \times \mathbb{P}^{1} \mid y t-z s=0\right\}
$$

é o blowup de $\mathbb{A}^{2}$ em $(0,0)$ com morfismo $\pi^{\prime}: X_{x} \rightarrow \mathbb{A}^{2}$ sendo a projeção natural em $\mathbb{A}^{2}$.

Por sua vez, podemos cobrir $X_{x}$ com os abertos $X_{x, s}=X_{x} \backslash \mathcal{Z}(s)$ e $X_{x, t}=X_{x} \backslash \mathcal{Z}(t)$. Veja que

$$
X_{x, s} \cong\left\{(y, z, t) \in \mathbb{A}^{3} \mid y t-z=0\right\}
$$

e que, nesse aberto, $\mathcal{E}=\pi^{-1}(P)$ satisfaz $\mathcal{E} \cap X_{x, s}=\mathcal{Z}(y) \cap X_{x, s}$.

O morfismo $\mu: X_{x, s} \rightarrow \mathbb{A}^{2}$ tal que $(y, z, t) \mapsto(y, t)$ e o morfismo inverso $\nu: \mathbb{A}^{2} \rightarrow X_{x, s}$ tal que $(y, t) \mapsto(y, y t, t)$ mostram que $X_{x, s}$ é isomorfo a $\mathbb{A}^{2}$. Com essa identificação, a restrição $\left.\pi^{\prime}\right|_{X_{x, s}}: X_{x, s} \rightarrow \mathbb{A}^{2}$ se descreve por $(y, t) \mapsto(y, y t)$.

Analogamente, teremos $X_{x, t}$ isomorfo a $\mathbb{A}^{2}$ e $\mathcal{E} \cap X_{x, t}=\mathcal{Z}(z) \cap X_{x, t}$. Com isso, conseguimos cobrir $X$ com 4 abertos $X_{y}, X_{z}, X_{x, s}$ e $X_{x, t}$ isomorfos a $\mathrm{A}^{2}$, que chamamos de cartas afins, e serão úteis para algumas contas. Para começar, isso já mostra que $X$ é irredutível e também não-singular.

Também podemos encontrar a transformada estrita de uma reta $L \subset \mathbb{A}^{2}$. Se $(0,0) \notin L$, então

$$
\widetilde{L}=\overline{\pi^{\prime-1}(L \backslash\{(0,0)\})}
$$

é simplesmente a pré-imagem de $L$ pelo morfismo $\pi^{\prime}$, ou seja $\widetilde{L}=\pi^{\prime-1}(L)$.

Se $(0,0) \in L$, podemos dizer que $L=\mathcal{Z}(a y-b z)$ para algum ponto $(a: b) \in \mathbb{P}^{1}$. Suponha sem perdas que $b \neq 0$ e usando que $\left.\pi^{\prime}\right|_{X_{x, s}}: X_{x, s} \rightarrow \mathbb{A}^{2}$ se descreve por $(y, t) \mapsto(y, y t)$, temos que

$$
\pi^{\prime-1}(L)=\mathcal{Z}(a y-b y t)=\mathcal{Z}(y) \cup \mathcal{Z}(a-b t)
$$


Como vimos que $\mathcal{E}=\mathcal{Z}(y)$ nesse aberto, podemos concluir que $\widetilde{L}=\mathcal{Z}(a-b t)$, uma reta em $X_{x, s}$. Então, a interseção $\widetilde{L} \cap \mathcal{E}$ corresponde ao ponto $\left(0, \frac{a}{b}\right)$ de $X_{x, s}$.

\section{2}

\section{$\mathrm{O}$ blowup de $\mathbb{P}^{2}$ em mais pontos}

Após tomar o blowup de $\mathbb{P}^{2}$ em um ponto $P$, podemos explodir a nova superfície em algum outro ponto, e assim sucessivamente. Uma descrição global para uma superfície assim obtida se torna cada vez mais complicada, de modo que preferiremos uma abordagem local.

Usaremos a notação $\mathbb{P}_{P_{0} \ldots P_{n}}^{2}$ para representar a superfície resultante após explodir $\mathbb{P}^{2}$ sucessivamente nos pontos $P_{0}, \ldots, P_{n} \in \mathbb{P}^{2}$ distintos. Mais precisamente, sejam $\pi_{0}: \mathbb{P}_{P_{0}}^{2} \rightarrow \mathbb{P}^{2}$ o blowup de $\mathbb{P}^{2}$ em $P_{0}$ e para cada $i=1, \ldots, n$ o blowup $\pi_{i}: \mathbb{P}_{P_{0} \ldots P_{i}}^{2} \rightarrow \mathbb{P}_{P_{0} \ldots P_{i-1}}^{2}$ de $\mathbb{P}_{P_{0} \ldots P_{i-1}}^{2}$ no ponto $\pi_{i-1}^{-1}\left(\ldots\left(\pi_{0}^{-1}\left(P_{i}\right)\right)\right)$, vamos considerar a composição

$$
\pi=\pi_{0} \circ \pi_{1} \circ \ldots \circ \pi_{n}: \mathbb{P}_{P_{0} \ldots P_{n}}^{2} \rightarrow \mathbb{P}^{2}
$$

Note que a princípio a ordem com que explodimos os $n+1$ pontos parece relevante.

Porém, se $\mathcal{E}_{i}=\pi^{-1}\left(P_{i}\right) \subset \mathbb{P}_{P_{0} \ldots P_{n}}^{2}$, então a superfície $\mathbb{P}_{P_{0} \ldots P_{n}}^{2}$ é uma modificação de $\mathbb{P}^{2}$, onde substituímos cada $P_{i}$ por $\mathcal{E}_{i} \cong \mathbb{P}^{1}$. Ou seja, podemos cobrir $\mathbb{P}_{P_{0} \ldots P_{n}}^{2}$ com abertos $U_{l}=\mathbb{P}_{P_{0} \ldots P_{n}}^{2} \backslash\left(\bigcup_{i \neq l} \mathcal{E}_{i}\right), l=0, \ldots, n$, sendo que cada $U_{l}$ isomorfo a $\mathbb{P}_{P_{l}}^{2} \backslash\left(\bigcup_{i \neq l}\left\{P_{i}\right\}\right)$.

Em outras palavras, a superfície $\mathbb{P}_{P_{0} \ldots P_{n}}^{2}$ é naturalmente uma colagem dos $n+1$ blowups $\mathbb{P}_{P_{0}}^{2}, \ldots, \mathbb{P}_{P_{n}}^{2}$ identificando ponto a ponto as pré-imagens de $\mathbb{P}^{2} \backslash\left\{P_{0}, \ldots, P_{n}\right\}$ pelos respectivos morfismos em $\mathbb{P}^{2}$. Mais precisamente, se $\tau_{0}: \mathbb{P}_{P_{0}}^{2} \rightarrow \mathbb{P}^{2}, \ldots, \tau_{n}: \mathbb{P}_{P_{n}}^{2} \rightarrow \mathbb{P}^{2}$ são os blowups de $\mathbb{P}^{2}$ em $P_{0}, \ldots, P_{n}$ respectivamente,

$$
\mathbb{P}_{P_{0} \ldots P_{n}}^{2}=\frac{\bigcup_{i=0, \ldots, n} \mathbb{P}_{P_{i}}^{2}}{\sim}
$$

onde $Q \in \mathbb{P}_{P_{l}}^{2}$ e $R \in \mathbb{P}_{P_{j}}^{2}$ satisfazem $Q \sim R$ quando $(Q, l)=(R, j)$ ou $\tau_{l}(Q)=\tau_{j}(R) \notin\left\{P_{0}, \ldots, P_{n}\right\}$.

Note que não é óbvio que essa colagem pode ser vista como uma variedade quasi-projetiva, mas, ao assumir a existência de $\mathbb{P}_{P_{0} \ldots P_{n}}^{2}$ como uma variedade quasi-projetiva, a identificação acima é correta. E desta forma, podemos ver que a ordem com que explodimos os $n+1$ pontos não muda a estrutura intrínseca do blowup final.

Vale lembrar que também podemos querer explodir $\mathbb{P}_{P_{0} \ldots P_{n}}^{2}$ em um ponto em algum dos divisores excepcionais $\mathcal{E}_{i}$. Neste caso, a colagem seria diferente, 
mas vamos deixar esta discussão para depois.

\section{3}

\section{Resolução de indeterminação}

Agora veremos como podemos transformar aplicações racionais e birracionais em morfismos usando blowups, e no futuro isso nos permitirá entender melhor os elementos de $\operatorname{Cr}\left(\mathbb{P}^{2}\right)$.

Teorema 4.2. Seja $X_{0}$ uma superfície projetiva complexa não-singular e $\varphi: X_{0} \rightarrow \mathbb{P}^{n}$ uma aplicação racional. Então existe uma cadeia finita de blowups $\pi_{i}: X_{i+1} \rightarrow X_{i}, i=0,1, \ldots, m$, de forma que a composição $\widetilde{\varphi}=\varphi \circ \pi_{0} \circ \ldots \circ \pi_{m}$ : $X_{m+1} \rightarrow \mathbb{P}^{n}$ é um morfismo.

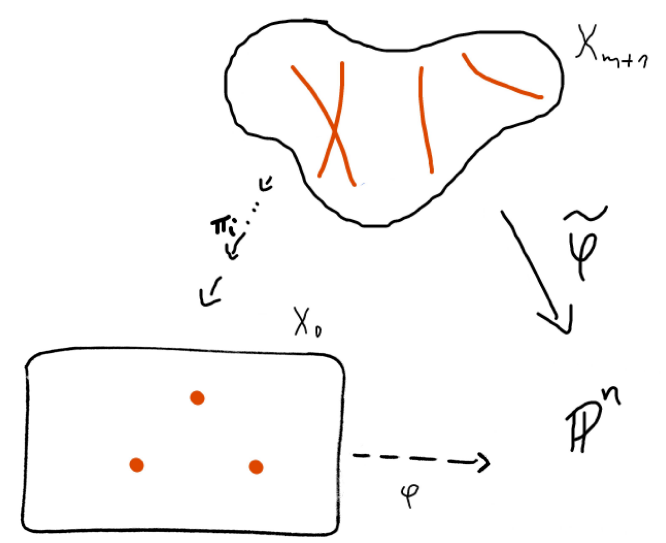

Este Teorema está na referência do Capítulo como Teorema 4.8.

Em outras palavras, a aplicação racional $\varphi$ pode ser composta com blowups de modo a eliminar sua indeterminação, processo conhecido como resolução de indeterminação.

Exemplo 20. A projeção linear de $\mathbb{P}^{2}$ no ponto $P=(1: 0: 0)$ é a aplicação racional $\varphi: \mathbb{P}^{2} \rightarrow \mathbb{P}^{1}$ definida por $(x: y: z) \mapsto(y: z)$ e tem um único ponto de indeterminação $P$. Ao tomar o blowup $\pi: \mathbb{P}_{P}^{2} \rightarrow \mathbb{P}^{2}$, o morfismo $\widetilde{\varphi}: \mathbb{P}_{P}^{2} \rightarrow \mathbb{P}^{1}$ $\operatorname{com}(x: y: z ; s: t) \mapsto(s: t)$ é equivalente como aplicação racional à composição $\varphi \circ \pi$. 


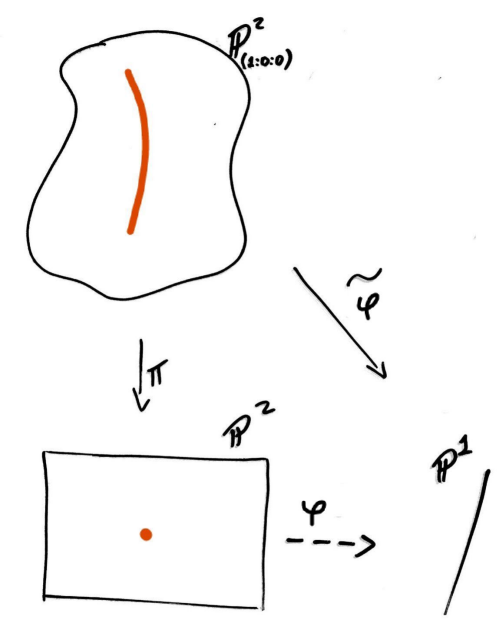

Exemplo 21. A Transformação Quadrática Padrão $\Phi: \mathbb{P}^{2} \rightarrow \mathbb{P}^{2}$ definida por $(x: y: z) \mapsto(y z: x z: x y)$ possui indeterminação nos três pontos coordenados $P_{0}=(1: 0: 0), P_{1}=(0: 1: 0)$ e $P_{2}=(0: 0: 1)$. Seja $L_{i j}$ a reta que liga os pontos $P_{i}$ e $P_{j}$ para $\{i, j\} \subset\{1,2,3\}$, sabemos que $\Phi$ é um automorfismo de

$$
A=\mathbb{P}^{2} \backslash\left(L_{01} \cup L_{02} \cup L_{12}\right)
$$

Ao tomar o blowup $\pi_{0}: \mathbb{P}_{P_{0}}^{2} \rightarrow \mathbb{P}^{2}$ de $\mathbb{P}^{2}$ em $P_{0}$, podemos compor com $\Phi$ e obter a aplicação racional $\Phi \circ \pi_{0}: \mathbb{P}_{P_{0}}^{2} \rightarrow \mathbb{P}^{2}$ tal que

$$
(x: y: z ; s: t) \mapsto(z s: x t: x s)=(y t: x t: x s) .
$$

Se $\Phi \circ \pi_{0}$ possui pontos de indeterminação, eles necessariamente estão nas pré-imagens de $P_{0}, P_{1}$ e $P_{2}$ por $\pi_{0}$. De fato, é fácil verificar que $\widetilde{P}_{1}=\pi_{0}^{-1}\left(P_{1}\right)=$ $(0: 1: 0 ; 1: 0)$ e $\widetilde{P}_{2}=\pi_{0}^{-1}\left(P_{2}\right)=(0: 0: 1 ; 0: 1)$ são ainda pontos de indeterminação. Porém, qualquer ponto $(1: 0: 0 ; s: t) \in E=\pi_{0}^{-1}\left(P_{0}\right)$ é levado em $(0: t: s) \in \mathbb{P}^{2}$, que está bem definido. Note que $E$ é levado bijetivamente em $L_{12}=\mathcal{Z}(x) \subset \mathbb{P}^{2}$.

Isso significa que $\Phi \circ \pi_{0}$ é um morfismo de $\mathbb{P}_{P_{0}}^{2} \backslash\left\{\widetilde{P}_{1}, \widetilde{P}_{2}\right\}$ em $\mathbb{P}^{2}$, e como aplicação racional agora possiu apenas dois pontos de indeterminação. Ou seja, ao tomar o blowup de $\mathbb{P}^{2}$ em um dos polos, resolvemos sua indeterminação naquele ponto.

Para resolvermos todas as indeterminações de $\Phi$, teremos que realizar mais blowups. Mais precisamente, vamos tomar o blowup $\pi_{1}: \mathbb{P}_{P_{0} P_{1}}^{2} \rightarrow \mathbb{P}_{P_{0}}^{2}$ de $\mathbb{P}_{P_{0}}^{2}$ em $\widetilde{P}_{1}$ e então o blowup $\pi_{2}: \mathbb{P}_{P_{0} P_{1} P_{2}}^{2} \rightarrow \mathbb{P}_{P_{0} P_{1}}^{2}$ de $\mathbb{P}_{P_{0} P_{1}}^{2}$ em $\pi_{1}^{-1}\left(\widetilde{P}_{2}\right)$. Essa composição de blowups é na verdade o blowup $\mathbb{P}_{P_{0} P_{1} P_{2}}^{2}$ de $\mathbb{P}^{2}$ em $P_{0}, P_{1}, P_{2}$, e denotamos a composição dos blowups por $\pi=\pi_{0} \circ \pi_{1} \circ \pi_{2}: \mathbb{P}_{P_{0} P_{1} P_{2}}^{2} \rightarrow \mathbb{P}^{2}$. 


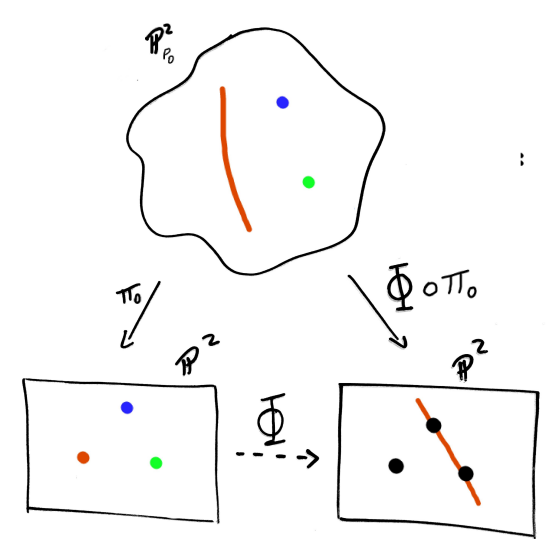

Dessa forma, se $\mathcal{E}_{0}=\pi^{-1}\left(P_{0}\right), \mathcal{E}_{1}=\pi^{-1}\left(P_{1}\right), \mathcal{E}_{2}=\pi^{-1}\left(P_{2}\right)$ como na subseção anterior, então $\pi_{1} \circ \pi_{2}$ restrito ao aberto $\mathbb{P}_{P_{0} P_{1} P_{2}}^{2} \backslash\left(\mathcal{E}_{1} \cup \mathcal{E}_{2}\right)$ é isomorfismo sobre a imagem $\mathbb{P}_{P_{0}}^{2} \backslash\left\{\widetilde{P}_{1}, \widetilde{P}_{2}\right\}$, que é o domínio de definição de $\Phi \circ \pi_{0}$. Ou seja, $\Phi \circ \pi_{0} \circ \pi_{1} \circ \pi_{2}$ é um morfismo de $\mathbb{P}_{P_{0} P_{1} P_{2}}^{2} \backslash\left(\mathcal{E}_{1} \cup \mathcal{E}_{2}\right)$ em $\mathbb{P}^{2}$, e como aplicação racional em $\mathbb{P}_{P_{0} P_{1} P_{2}}^{2}$ seu conjunto de indeterminação está contido em $\mathcal{E}_{1} \cup \mathcal{E}_{2}$.

De forma análoga, seria possível provar que seu conjunto de indeterminação está contido em $\mathcal{E}_{0} \cup \mathcal{E}_{2}$ e em $\mathcal{E}_{0} \cup \mathcal{E}_{1}$, e portanto $\Phi \circ \pi_{0} \circ \pi_{1} \circ \pi_{2}$ é na verdade um morfismo em $\mathbb{P}_{P_{0} P_{1} P_{2}}^{2}$.

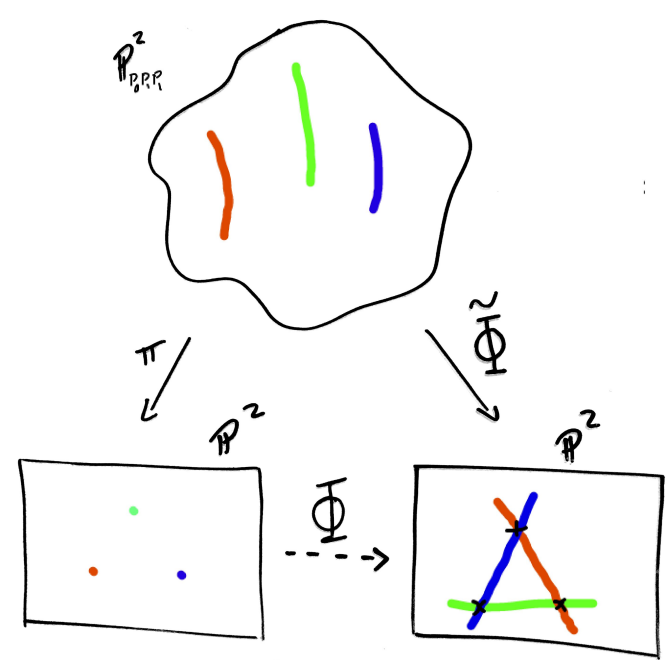


Teorema 4.3. Sejam $X_{0}$ e $Y_{0}$ superfícies projetivas não-singulares e $\varphi$ : $X_{0} \rightarrow Y_{0}$ uma aplicação birracional. Então existe uma cadeia finita de blowups $\pi_{i}: X_{i+1} \rightarrow X_{i}, i=0,1, \ldots, m$, e outra cadeia finita de blowups $\tau_{j}: Y_{j+1} \rightarrow Y_{j}$, $j=0,1, \ldots, l$, de forma que $X_{m+1} \cong Y_{l+1}$ e a composição $\varphi \circ \pi_{0} \circ \ldots \circ \pi_{m}: X_{m+1} \rightarrow$ $Y_{0}$ equivale como aplicação racional ao morfismo $\tau_{0} \circ \ldots \circ \tau_{l}: Y_{l+1} \rightarrow Y_{0}$.

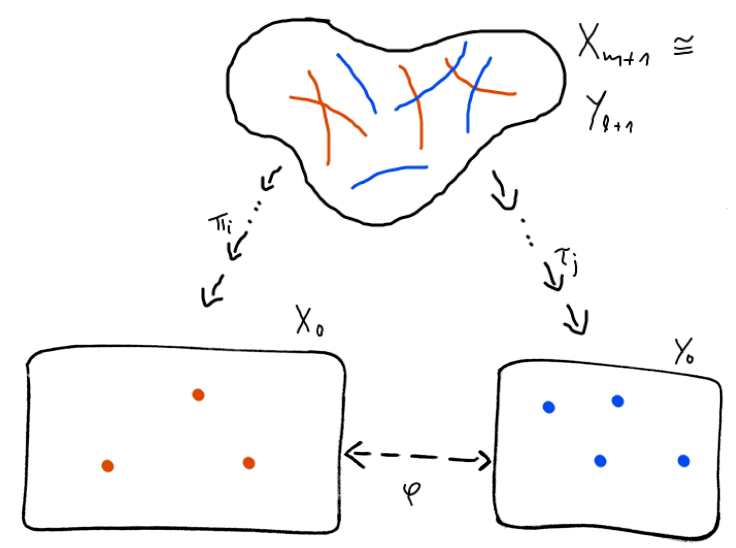

Este Teorema está na referência do Capítulo como Teorema 4.9

Exemplo 22. Seguindo do exemplo anterior, temos que a Transformação Quadrática Padrão $\Phi: \mathbb{P}^{2} \rightarrow \mathbb{P}^{2}$ admite uma resolução de indeterminação $\widetilde{\Phi}=$ $\Phi \circ \pi_{0} \circ \pi_{1} \circ \pi_{2}: \mathbb{P}_{P_{0} P_{1} P_{2}}^{2} \rightarrow \mathbb{P}^{2}$. Veremos que o morfismo $\widetilde{\Phi}$ é a composição de três explosões de $\mathbb{P}^{2}$.

Sabemos que $\Phi$ induz um automorfismo de $A=\mathbb{P}^{2} \backslash\left(L_{01} \cup L_{02} \cup L_{12}\right)$ e que $\pi_{0}$ induz um isomorfismo de $\pi_{0}^{-1}(A)$ em $A$, portanto a composição $\Phi \circ \pi_{0}$ induz um isomorfismo

$$
\pi_{0}^{-1}(A)=\mathbb{P}_{P_{0}}^{2} \backslash\left(E \cup \widetilde{L_{01}} \cup \widetilde{L_{02}} \cup \widetilde{L_{12}}\right) \stackrel{\sim}{\rightarrow} A,
$$

onde cada $\widetilde{L_{i j}}$ é a transformada estrita da reta $L_{i j}$ por $\pi_{0}$. Mas dado que $\Phi \circ \pi_{0}$ leva $E$ em $L_{12}$ bijetivamente, faz sentido tentarmos verificar que $\Phi \circ \pi_{0}$ induz um isomorfismo

$$
\mathbb{P}_{P_{0}}^{2} \backslash\left(\widetilde{L_{01}} \cup \widetilde{L_{02}} \cup \widetilde{L_{12}}\right) \stackrel{\sim}{\rightarrow} \mathbb{P}^{2} \backslash\left(L_{01} \cup L_{02}\right)
$$

E de fato, a aplicação racional inversa $\left(\Phi \circ \pi_{0}\right)^{-1}: \mathbb{P}^{2} \rightarrow \mathbb{P}_{P_{0}}^{2}$ é dada por $(x: y: z) \mapsto(y z: x z: x y ; z: y)$, e leva $L_{12} \backslash\left\{P_{1}, P_{2}\right\}$ bijetivamente em $E \backslash\left\{\widetilde{P}_{1}, \widetilde{P}_{2}\right\}$, então temos exatamente o que queríamos.

Consideremos também, que após explodirmos $\mathbb{P}_{P_{0}}^{2}$ mais duas vezes pelo morfismo $\pi_{1} \circ \pi_{2}$, temos o isomorfismo induzido

$$
\mathbb{P}_{P_{0} P_{1} P_{2}}^{2} \backslash\left(\mathcal{E}_{1} \cup \mathcal{E}_{2} \cup \widetilde{\widetilde{L_{01}}} \cup \widetilde{\widetilde{L_{02}}} \cup \widetilde{\widetilde{L_{12}}}\right) \stackrel{\sim}{\rightarrow} \mathbb{P}_{P_{0}}^{2} \backslash\left(\widetilde{L_{01}} \cup \widetilde{L_{02}} \cup \widetilde{L_{12}}\right),
$$




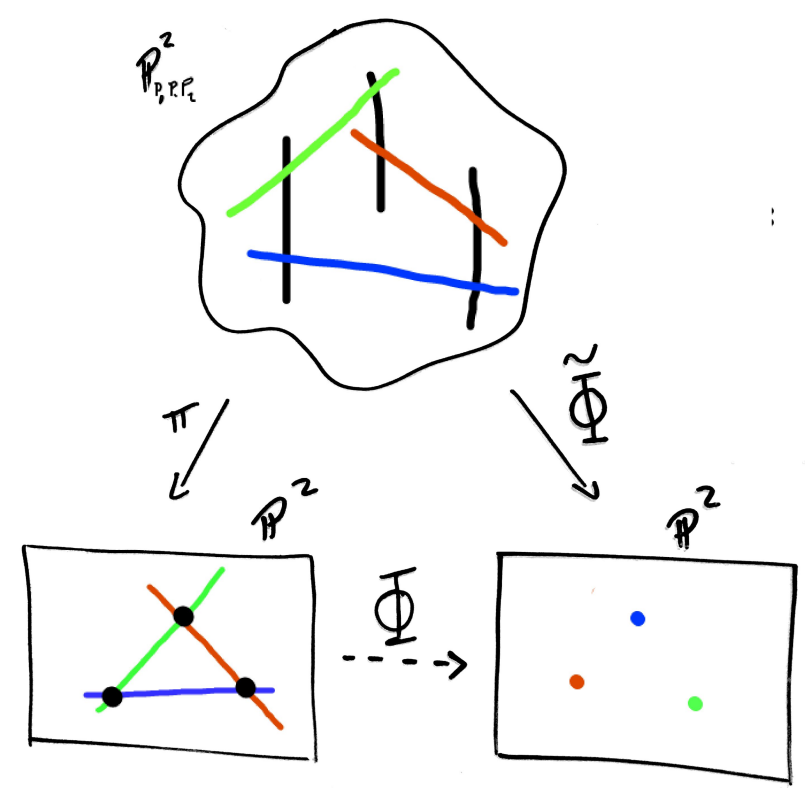

onde cada $\widetilde{\widetilde{L_{i j}}}$ é a transformada estrita de $\widetilde{L_{i j}}$ por $\pi_{1} \circ \pi_{2}$

Finalmente, a composição $\Phi \circ \pi_{0} \circ \pi_{1} \circ \pi_{2}$ induz um isomorfismo

$$
\mathbb{P}_{P_{0} P_{1} P_{2}}^{2} \backslash\left(\mathcal{E}_{1} \cup \mathcal{E}_{2} \cup \widetilde{\widetilde{L_{01}}} \cup \widetilde{\widetilde{L_{02}}} \cup \widetilde{\widetilde{L_{12}}}\right) \stackrel{\sim}{\rightarrow} \mathbb{P}^{2} \backslash\left(L_{01} \cup L_{02}\right)
$$

De forma análoga, também ocorrem os isomorfismos

$$
\begin{aligned}
& \mathbb{P}_{P_{0} P_{1} P_{2}}^{2} \backslash\left(\mathcal{E}_{0} \cup \mathcal{E}_{2} \cup \widetilde{\widetilde{L_{01}}} \cup \widetilde{\widetilde{L_{02}}} \cup \widetilde{\widetilde{L_{12}}}\right) \stackrel{\sim}{\rightarrow} \mathbb{P}^{2} \backslash\left(L_{01} \cup L_{12}\right), \\
& \mathbb{P}_{P_{0} P_{1} P_{2}}^{2} \backslash\left(\mathcal{E}_{0} \cup \mathcal{E}_{1} \cup \widetilde{\widetilde{L_{01}}} \cup \widetilde{\widetilde{L_{02}}} \cup \widetilde{\widetilde{L_{12}}}\right) \stackrel{\sim}{\rightarrow} \mathbb{P}^{2} \backslash\left(L_{02} \cup L_{12}\right)
\end{aligned}
$$

induzidos pela mesma aplicação racional $\Phi \circ \pi_{0} \circ \pi_{1} \circ \pi_{2}$, portanto podemos concluir que tal aplicação racional induz um isomorfismo

$$
\mathbb{P}_{P_{0} P_{1} P_{2}}^{2} \backslash\left(\widetilde{\widetilde{L_{01}}} \cup \widetilde{\widetilde{L_{02}}} \cup \widetilde{\widetilde{L_{12}}}\right) \stackrel{\sim}{\rightarrow} \mathbb{P}^{2} \backslash\left\{P_{0}, P_{1}, P_{2}\right\}
$$

Além disso, a imagem de cada transformada estrita $\widetilde{\widetilde{L_{i j}}}$ por $\Phi \circ \pi_{0} \circ \pi_{1} \circ \pi_{2}$ é simplsmente $\Phi\left(L_{i j}\right)=P_{k}$ para $\{i, j, k\}=\{0,1,2\}$. Portanto temos que $\Phi \circ \pi_{0} \circ \pi_{1} \circ \pi_{2}$ é na verdade a explosão de $\mathbb{P}^{2}$ nos pontos $P_{0}, P_{1}, P_{2}$ cujos

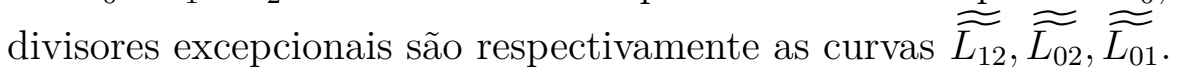




\section{5}

\section{Divisores}

A toda variedade projetiva não-singular $X$ podemos associar um grupo, chamado Grupo de Picard de $X$. Os Grupos de Picard de explosões de $\mathbb{P}^{2}$ podem nos ajudar a entender os elementos de $\operatorname{Cr}\left(\mathbb{P}^{2}\right)$. Para defini-lo, precisamos antes conhecer o Grupo de Divisores de $X$ e quocientá-lo por um subgrupo especial, o subgrupo dos divisores principais de $X$. Para referência geral do Capítulo, verificar (SHAFAREVICH, 1994) Capítulos 3.1.1, 3.1.2, 3.1.3 e 3.1.4.

\section{1}

\section{O Grupo de Picard}

Seja $X$ uma variedade quasi-projetiva não-singular.

Definição 5.1. Um divisor primo $Z$ de $X$ é um fechado irredutivel $Z \subset X$ de codimensão 1. Seja Div $(X)^{p}$ o conjunto dos divisores primos de $X$.

Exemplo 23. É fácil verificar que, como conjunto, $\operatorname{Div}\left(\mathbb{P}^{1}\right)^{p}=\mathbb{P}^{1}$ e $\operatorname{Div}\left(\mathbb{P}^{2}\right)^{p}=$ \{curvas irredutíveis de $\left.\mathbb{P}^{2}\right\}$.

Exemplo 24. Seja $\pi: S_{P} \rightarrow S$ a explosão de uma superfície $S$ em um ponto $P \in S$, o divisor excepcional $\mathcal{E}=\pi^{-1}(P)$ é um divisor primo de $S_{P}$.

Qualquer outro divisor primo $Z \subset S_{P}$ tem interseção finita com $\mathcal{E}$, e portanto $Z \backslash \mathcal{E}$ é irredutível em $S_{P} \backslash \mathcal{E}$. Como $Z \backslash \mathcal{E}$ é isomorfo a $\pi(Z) \backslash\{P\}$, então $\pi(Z)=C \subset S$ é uma curva irredutível. Nesse caso, $Z=\widetilde{C}$.

Em outras palavras,

$$
\operatorname{Div}\left(S_{P}\right)^{p}=\left\{\widetilde{C} \mid C \in \operatorname{Div}(S)^{p}\right\} \cup\{\mathcal{E}\}
$$

é composto pelas transformadas estritas $\widetilde{C}$ de curvas irredutíveis $C$ de $S$ juntamente com o divisor excepcional $\mathcal{E}$.

Nosso interesse pelos divisores primos de uma variedade se dá por serem bem entendidos, comparados a outros fechados da variedade. Mais precisamente, vamos manter em mente o seguinte Teorema como motivação ((SHAFAREVICH, 1994) Teorema 2.10).

Teorema 5.1. Seja $Z \subset X$ um divisor primo de $X$. Então para todo ponto $P \in X$ existe uma vizinhança aberta $U \subset X$ de $P \operatorname{com} U \cap Z \neq \varnothing$ e uma função $\pi \in \mathcal{O}(U)$ regular em $U$ tal que $Z \cap U=\mathcal{Z}(\pi) \subset U$, e mais ainda, o ideal

$$
\mathcal{I}_{U}(Z)=\{f \in \mathcal{O}(U) \mid f(Q)=0 \forall Q \in Z \cap U\}
$$


é principal com $\mathcal{I}_{U}(Z)=\langle\pi\rangle \subset \mathcal{O}(U)$.

Definição 5.2. Um divisor $D$ de $X$ é uma soma formal de divisores primos de $X$, ou seja,

$$
D=\sum_{Z \in D i v(X)^{p}} a_{Z} Z
$$

com $a_{Z} \in \mathbb{Z}$ para todo $Z \in \operatorname{Div}(X)^{p}$ e $a_{Z} \neq 0$ para finitos $Z$ 's. Chamaremos de $\operatorname{Div}(X)$ o grupo de divisores de $X$ com a operação natural de soma. Este é o menor grupo abeliano contendo os divisores primos de $X$.

O suporte de um divisor $D$ qualquer é a união dos divisores primos na sua soma, isto é,

$$
\operatorname{Supp}(D)=\bigcup_{a_{Z} \neq 0} Z \subset X .
$$

Agora queremos associar a cada função racional em $X$ um divisor, que descreva exatamente ao longo de quais divisores primos essa função se anula e ao longo de quais ela é um polo, contando multiplicidade. Para isso, vamos definir a ordem de anulamento de uma função racional ao longo de um divisor primo.

Definição 5.3. Dado $Z \in \operatorname{Div}(X)^{p}$ um divisor primo de $X$, sejam $U \subset X$ um aberto tal que $Z \cap U \neq \varnothing$ e $\pi \in \mathcal{O}(U)$ como no Teorema 5.1. Dada uma função $f \in \mathcal{O}(U)$ regular em $U$, definimos a ordem de anulamento de $f$ em $Z$ como o número inteiro $m \geq 0$ tal que $f \in \mathcal{I}_{U}(Z)^{m}=\left\langle\pi^{m}\right\rangle$ e $f \notin \mathcal{I}_{U}(Z)^{m+1}=\left\langle\pi^{m+1}\right\rangle$, denotado por $\operatorname{ord}_{Z}(f)$.

A definição não depende do aberto $U$. De fato, suponha que $U^{\prime} \subset X$ e $\pi^{\prime} \in \mathcal{O}\left(U^{\prime}\right)$ também satisfazem as condições do Teorema 5.1, com $f \in \mathcal{O}\left(U^{\prime}\right)$. Primeiramente, notemos que

$$
\left(U \cap U^{\prime}\right) \cap Z=(U \cap Z) \cap\left(U^{\prime} \cap Z\right)
$$

é a interseção de dois abertos densos em $Z$ e portanto é não vazia já que $Z$ é irredutível. Além disso, as restrições de $\pi$ e $\pi^{\prime}$ a $U \cap U^{\prime}$ geram cada uma o ideal $\mathcal{I}_{U \cap U^{\prime}}(Z)$, ou seja,

$$
\left\langle\left.\pi\right|_{U \cap U^{\prime}}\right\rangle=\mathcal{I}_{U \cap U^{\prime}}(Z)=\left\langle\left.\pi^{\prime}\right|_{U \cap U^{\prime}}\right\rangle
$$

Portanto temos o mesmo $m$ se considerarmos $f$ como uma função regular em qualquer um dos abertos $U, U \cap U^{\prime}$ e $U^{\prime}$.

Podemos verificar a seguinte propriedade: para todos $f, g \in \mathcal{O}(U)$ temos $\operatorname{ord}_{Z}(f g)=\operatorname{ord}_{Z}(f)+\operatorname{ord}_{Z}(g)$. Inspirados nela, podemos dar a seguinte generalização para a definição anterior. 
Definição 5.4. Dado $Z \in \operatorname{Div}(X)^{p}$ um divisor primo de $X$, sejam $U \subset X$ um aberto tal que $Z \cap U \neq \varnothing$ e $\pi \in \mathcal{O}(U)$ como no Teorema 5.1. Seja $f \in K(X)$ uma função racional de $X$ qualquer, podemos escrever $f=g / h$ com $g, h \in \mathcal{O}(U)$, e definimos a ordem de anulamento de $f$ em $Z$ como $\operatorname{ord}_{Z}(f)=\operatorname{ord}_{Z}(g)-\operatorname{ord}_{Z}(h)$.

Essa definição não depende da escolha de $g$ e $h$ e nem do aberto $U \subset X$, pois se $f=g / h=g^{\prime} / h^{\prime} \operatorname{com} g, h \in \mathcal{O}(U)$ e $g^{\prime}, h^{\prime} \in \mathcal{O}\left(U^{\prime}\right)$, então $g h^{\prime}=g^{\prime} h$ em $U \cap U^{\prime}$ e portanto $\operatorname{ord}_{Z}(g)+\operatorname{ord}_{Z}\left(h^{\prime}\right)=\operatorname{ord}_{Z}\left(g^{\prime}\right)+\operatorname{ord}_{Z}(h)$ olhando $g, g^{\prime}, h, h^{\prime}$ como funções regulares em $U \cap U^{\prime}$.

Dizemos que $f$ se anula ao longo de $Z$ quando $\operatorname{ord}_{Z}(f)$ é positiva e que $f$ tem polo ao longo de $Z$ quando é negativa, e seu valor absoluto representa a multiplicidade do zero ou do polo.

Definição 5.5. Seja $f \in K(X)$ não nula, definimos o divisor associado a $f$ como

$$
\operatorname{div}(f)=\sum_{Z \in \operatorname{Div}(X)^{p}} \operatorname{ord}_{Z}(f) Z
$$

É um fato de álgebra comutativa que para uma função racional $f$ fixa, $\operatorname{ord}_{Z}(f) \neq 0$ para finitos divisores primos $Z$, logo a definição 5.5 é uma soma finita e $\operatorname{div}(f) \in \operatorname{Div}(X)$.

Exemplo 25. Tome $X=\mathbb{P}^{2}$. Nesse caso, se $f \in K\left(\mathbb{P}^{2}\right)$ é não nula, podemos escrever $f=G / H$ como o quociente de polinômios homogêneos de mesmo grau sem fatores comuns $G, H \in k[x, y, z]$. Ao fatorar $G=\prod G_{i}^{m_{i}}$ e $H=\prod H_{j}^{n_{j}}$ em polinômios homogêneos irredutíveis, podemos encontrar

$$
\operatorname{div}(f)=\sum m_{i} \mathcal{Z}\left(G_{i}\right)-\sum n_{j} \mathcal{Z}\left(H_{j}\right) \in \operatorname{Div}(X)
$$

Exemplo 26. Seja $f \in K(X)$ não nula, então $\operatorname{div}(f)=0$ se e só se $f \in \mathcal{O}(X)$ e $f$ não se anula em nenhum ponto de $X$. Como ainda vale que para todos $f, g \in K(X)$ temos $\operatorname{ord}_{Z}(f g)=\operatorname{ord}_{Z}(f)+\operatorname{ord}_{Z}(g)$, podemos concluir que $\operatorname{div}(f)=\operatorname{div}(g)$ se e só se $f / g \in \mathcal{O}(X)$ e $f / g$ não se anula em nenhum ponto de $X$.

Definição 5.6. Dizemos que um divisor $D \in \operatorname{Div}(X)$ é principal quando existe uma função racional $f \in K(X)$ tal que $D=\operatorname{div}(f)$. Perceba que como temos $\operatorname{div}(f)+\operatorname{div}(g)=\operatorname{div}(f . g)$ para quaisquer $f, g \in K(X)$ não nulos, os divisores principais formam um subgrupo de Div $(X)$.

Dois divisores $D$ e $D^{\prime}$ são linearmente equivalentes e escrevemos $D \sim D^{\prime}$ quando $D-D^{\prime} \in \operatorname{Div}(X)$ é principal, ou seja, quando existe uma função racional $f \in K(X)$ tal que $\operatorname{div}(f)=D-D^{\prime}$. 
Exemplo 27. Quando $X=\mathbb{P}^{2}, K(X)=\{F / G \mid F, G \in k[x, y, z]$ homogêneos de mesmo grau, $G \neq 0\}$.

Dado um divisor $D=\sum n_{i} Z_{i}$, onde $Z_{i}=\mathcal{Z}\left(F_{i}\right)$ é o conjunto de zeros de um polinômio homogêneo irredutível $F_{i}$, podemos definir

$$
\operatorname{deg}(D)=\sum n_{i} \operatorname{deg}\left(F_{i}\right)
$$

como uma generalização de grau de uma curva.

Note que se $D$ for um divisor principal, então

$$
D=\sum m_{i} Z\left(G_{i}\right)-\sum n_{j} Z\left(H_{j}\right)
$$

onde $G=\prod G_{i}^{m_{i}}$ e $H=\prod H_{j}^{n_{j}}$ são polinômios homogêneos de mesmo grau, e portanto

$$
\operatorname{deg}(D)=\sum m_{i} \operatorname{deg}\left(G_{i}\right)-\sum n_{j} \operatorname{deg}\left(H_{j}\right)=\operatorname{deg}(G)-\operatorname{deg}(H)=0
$$

Reciprocamente, se $D \in \operatorname{Div}\left(\mathbb{P}^{2}\right)$ tem grau $\operatorname{deg}(D)=0$, então $D$ é principal. De fato, se

$$
\operatorname{deg}(D)=\sum n_{i} \operatorname{deg}\left(F_{i}\right)=\sum_{n_{i}>0} n_{i} \operatorname{deg}\left(F_{i}\right)-\sum_{n_{i}<0}\left(-n_{i}\right) \operatorname{deg}\left(F_{i}\right)=0
$$

podemos escrever

$$
D=\operatorname{div}\left(\frac{\prod_{n_{i}>0} F_{i}^{n_{i}}}{\prod_{n_{i}<0} F_{i}^{-n_{i}}}\right)
$$

como o divisor de um quociente de polinômios homogêneos de mesmo grau.

Sendo assim, como a nossa função $\operatorname{deg}: \operatorname{Div}\left(\mathbb{P}^{2}\right) \rightarrow \mathbb{Z}$ é um homomorfismo de grupos sobrejetor, dois divisores $D, D^{\prime} \in \operatorname{Div}\left(\mathbb{P}^{2}\right)$ são linearmente equivalentes se e só se $\operatorname{deg}(D)=\operatorname{deg}\left(D^{\prime}\right)$.

Definição 5.7. Finalmente, definimos o Grupo de Picard de uma variedade quasi-projetiva não-singular $X$ como

$$
\operatorname{Pic}(X)=\operatorname{Div}(X) /\{\text { divisores principais }\}=\operatorname{Div}(X) / \sim \text {. }
$$

Exemplo 28. Podemos concluir do exemplo anterior que todos os divisores de mesmo grau são linearmente equivalentes e $\operatorname{Pic}\left(\mathbb{P}^{2}\right) \cong \mathbb{Z}$. De forma análoga vemos que $\operatorname{Pic}\left(\mathbb{P}^{n}\right) \cong \mathbb{Z}$ para qualquer inteiro positivo $n$.

Exemplo 29. Também é possível verificar que dois divisores $D, D^{\prime} \in \operatorname{Div}\left(\mathbb{P}^{n} \times\right.$ $\left.\mathbb{P}^{m}\right)$ são linearmente equivalentes se e só se possuem o mesmo bi-grau. Portanto $\operatorname{Pic}\left(\mathbb{P}^{n} \times \mathbb{P}^{m}\right) \cong \mathbb{Z}^{2}$. 
Exemplo 30. Mais tarde veremos que $\operatorname{Pic}\left(\mathbb{P}_{P}^{2}\right) \cong \mathbb{Z}^{2}$, e mais geralmente que $\operatorname{Pic}\left(\mathbb{P}_{P_{0} \ldots P_{n-1}}^{2}\right) \cong \mathbb{Z}^{n+1}$.

\section{2}

\section{O pullback de divisores}

Definição 5.8. Seja $Y$ uma variedade projetiva não-singular, $V \subset Y$ um aberto não vazio e $Z \in \operatorname{Div}(Y)^{p}$ um divisor primo. Definimos a restrição de $Z$ a $V$ como $\left.Z\right|_{V}=Z \cap V \in \operatorname{Div}(V)^{p}$, se $V \cap Z \neq \varnothing$ ou 0 se $V \cap Z=\varnothing$. Mais geralmente, se

$$
D=\sum_{Z \in \operatorname{Div}(Y)^{p}} a_{Z} Z \in \operatorname{Div}(Y)
$$

é um divisor qualquer, sua restrição a $V$ é

$$
\left.D\right|_{V}=\left.\sum_{Z \in \operatorname{Div}(Y)^{p}} a_{Z} Z\right|_{V} \in \operatorname{Div}(V) .
$$

Vale observar que teremos facilmente que se $V=Y \backslash W$ onde $W \subset Y$ é um fechado de codimensão pelo menos 2 , então $\operatorname{Div}(V) \cong \operatorname{Div}(Y)$. Como também sabemos que $K(V) \cong K(Y)$ nos dá uma identificação bijetiva entre o conjunto de divisores principais de $V$ e o conjunto de divisores principais de $Y$, teremos $\operatorname{Pic}(V) \cong \operatorname{Pic}(X)$.

Porém, quando $W$ tem codimensão 1 , suas componentes irredutíveis são divisores primos de $Y$ que não intersectam $V=Y \backslash W$. Ou seja, existe uma inclusão de $\operatorname{Div}(V)$ em $\operatorname{Div}(Y)$ tomando o fecho de cada divisor primo, mas que não é sobrejetiva.

Exemplo 31. Dada uma superfície $S$ e um ponto $P \in S$ qualquer, então $\operatorname{Pic}(S) \cong \operatorname{Pic}(S \backslash P)$. Esse detalhe será muito importante quando quisermos entender o grupo de Picard do blowup de $S$ em $P$.

Afinal, se $\pi: S_{P} \rightarrow S$ é o blowup se $S$ em $P$ com divisor excepcional $\mathcal{E}=\pi^{-1}(P)$, temos $S \backslash P \cong S_{P} \backslash \mathcal{E}$ e por isso

$$
\operatorname{Div}\left(S_{P}\right) \cong \operatorname{Div}\left(S_{P} \backslash \mathcal{E}\right) \oplus \mathbb{Z} \mathcal{E} \cong \operatorname{Div}(S) \oplus \mathbb{Z} \mathcal{E}
$$

Por construção, a inclusão $\operatorname{Div}(S) \hookrightarrow \operatorname{Div}\left(S_{P}\right)$ se dá usando a transformada estrita da explosão.

Mais tarde, poderemos provar também que

$$
\operatorname{Pic}\left(S_{P}\right) \cong \operatorname{Pic}\left(S_{P} \backslash \mathcal{E}\right) \oplus \mathbb{Z}[\mathcal{E}] \cong \operatorname{Pic}(S) \oplus \mathbb{Z}[\mathcal{E}]
$$


De fato, já podemos verificar o segundo isomorfismo, afinal

$$
\operatorname{Pic}\left(S_{P} \backslash \mathcal{E}\right) \cong \operatorname{Pic}(S \backslash P) \cong \operatorname{Pic}(S)
$$

Já o primeiro isomorfismo provaremos em breve.

O seguinte Teorema nos ajudará a construir o pullback de divisores por um morfismo sobrejetor.

Teorema 5.2. Seja Y uma variedade projetiva não-singular. Todo divisor $D \in \operatorname{Div}(Y)$ é localmente principal, ou seja, para todo ponto $P \in Y$ existe uma vizinhança aberta $V \subset Y$ de $P$ e uma função racional $f \subset K(V)$ tais que

$$
\left.D\right|_{V}=\operatorname{div}(f) .
$$

Prova. Seja $D=a_{1} Z_{1}+\ldots+a_{k} Z_{k}$ um divisor de $Y$. Dado $P \in Y$, para cada $i=1, \ldots, k$ considere um aberto $V_{i} \subset Y$ e uma função $\pi_{i} \in \mathcal{O}\left(V_{i}\right)$ como no Teorema 5.1. Então faça $V=\bigcap_{i=1}^{k} V_{i}$ e $f=\prod_{i=1}^{k} \pi_{i}^{a_{i}} \in K(V)$.

Observe que enquanto tratamos $f \in K(V)$ como função racional no aberto $V$, é mais conveniente em termos de notação escrever $\left.D\right|_{V}=\operatorname{div}(f)$. Porém, às vezes faz sentido tratar $f$ como uma função racional em todo $Y$, afinal $K(V) \cong K(Y)$. Nesse caso, precisaremos escrever $\left.D\right|_{V}=\left.\operatorname{div}(f)\right|_{V}$.

Exemplo 32. Seja $Y=\mathbb{P}^{2}$ e $L \subset \mathbb{P}^{2}$ uma reta, isto é, $L=\mathcal{Z}(F)$ para $F \in k[x, y, z]^{h}$ homogênea de grau 1 . Dado $P \in \mathbb{P}^{2}$ qualquer, tome uma outra reta $L^{\prime}=\mathcal{Z}\left(F^{\prime}\right) \subset \mathbb{P}^{2}$ de modo que $P \notin L^{\prime}$. Assim, se $V=\mathbb{P}^{2} \backslash L^{\prime} \subset \mathbb{P}^{2}$ e $f=F / F^{\prime} \in K\left(\mathbb{P}^{2}\right)$, temos

$$
\left.L\right|_{V}=\left.\operatorname{div}(f)\right|_{V}
$$

Considere $X, Y$ variedades projetivas não-singulares e $\pi: X \rightarrow Y$ um morfismo sobrejetor. Dado $D \in \operatorname{Div}(Y)$ um divisor de $Y$, gostaríamos de definir $\pi^{*} D$ o pullback de $D$ por $\pi$, que será um divisor de $X$.

Sabemos que o morfismo $\pi: X \rightarrow Y$ induz um monomorfismo de corpos $\pi^{*}: K(Y) \rightarrow K(X)$ com $f \mapsto f \circ \pi$, e portanto, para cada $V \subset Y$ e $f \in$ $K(V) \cong K(Y)$ como no Teorema 5.2 e $U=\pi^{-1}(V) \subset X$ aberto, podemos tomar $\pi^{*} f=f \circ \pi \in K(U)$ e definir $\pi^{*} D$ localmente, de forma que

$$
\left.\pi^{*} D\right|_{U}=\operatorname{div}\left(\pi^{*} f\right)
$$

Se por exemplo

$$
\operatorname{div}\left(\pi^{*} f\right)=\sum_{Z \in \operatorname{Div}(U)^{p}} a_{Z} Z
$$


podemos encontrar parte dos fatores de $\pi^{*} D$ na soma

$$
\sum_{Z \in \operatorname{Div}(U)^{p}} a_{Z} \bar{Z}
$$

onde $\bar{Z} \subset X$ é o fecho topológico de $Z$ em $X$. Porém, ainda podem existir divisores primos da soma de $\pi^{*} D$ contidos em $X \backslash U$, por isso fazemos uma colagem para recuperar $\pi^{*} D$ completamente.

É possível verificar que dados $V_{1}, V_{2} \subset Y$ distintos com $\left.D\right|_{V_{1}}=\operatorname{div}\left(f_{1}\right)$ e $\left.D\right|_{V_{2}}=\operatorname{div}\left(f_{2}\right)$, as restrições $\left.\pi^{*} D\right|_{U_{1}}$ e $\left.\pi^{*} D\right|_{U_{2}}$ são compatíveis, de forma que $\pi^{*} D$ está bem definido. De fato, como $\left.\operatorname{div}\left(f_{1}\right)\right|_{V_{1} \cap V_{2}}=\left.\operatorname{div}\left(f_{2}\right)\right|_{V_{1} \cap V_{2}}$, temos que $f_{1} / f_{2}$ é uma função regular em $V_{1} \cap V_{2}$ que não se anula em $V_{1} \cap V_{2}$. Portanto $\pi^{*} f_{1} / \pi^{*} f_{2}=\left(f_{1} / f_{2}\right) \circ \pi$ é uma função regular e que não se anula em $U_{1} \cap U_{2}$, e consequentemente $\left.\operatorname{div}\left(\pi^{*} f_{1}\right)\right|_{U_{1} \cap U_{2}}=\left.\operatorname{div}\left(\pi^{*} f_{2}\right)\right|_{U_{1} \cap U_{2}}$.

Observe que construímos um homomorfismo de grupos $\pi^{\star}: \operatorname{Div}(Y) \rightarrow$ $\operatorname{Div}(X)$ que leva divisores principais em divisores principais. Como consequência, temos a seguinte Proposição.

Proposição 5.1. O pullback preserva equivalência linear. Mais precisamente, se $\pi: X \rightarrow Y$ é um morfismo sobrejetor e $D, D^{\prime}$ são divisores de $Y$ tais que $D \sim D^{\prime}$, então $\pi^{*} D \sim \pi^{*} D^{\prime}$ como divisores de $X$. Portanto, o homomorfismo $\pi^{*}: \operatorname{Div}(Y) \rightarrow \operatorname{Div}(X)$ induz um homomorfismo nos grupos quocientes $\pi^{*}: \operatorname{Pic}(Y) \rightarrow \operatorname{Pic}(X)$.

Estaremos especialmente interessados em tomar o pullback de divisores por morfismos birracionais, que por sua vez podem ser decompostos em finitas explosões, pelo Teorema 4.1. Portanto gostaríamos especialmente de entender o que faz o pullback de divisores por uma explosão.

Proposição 5.2. Seja $\pi: S_{P} \rightarrow S$ a explosão de uma superfície quasi-projetiva $S$ no ponto $P \in S$ com divisor excepcional $\mathcal{E}=\pi^{-1}(P)$. Considere $C \in \operatorname{Div}(S)^{p}$ uma curva irredutivel e $f \in K(S)$ uma função racional quaisquer. Então

$$
\operatorname{ord}_{\widetilde{C}}(f \circ \pi)=\operatorname{ord}_{C}(f)
$$

Prova. Sejam $V \subset S$ aberto e $\varphi \in \mathcal{O}(V)$ função regular que define $C$ localmente como no Teorema 5.1. Seja $U=\pi^{-1}(V) \subset S_{P}$, é claro que a função regular composta $\varphi \circ \pi \in \mathcal{O}(U)$ define localmente a curva $\pi^{-1}(C)$, que pode não ser irredutível, mas possui $\widetilde{C}$ como uma componente irredutível. É fácil ver que em $U^{\prime}=U \backslash \mathcal{E}$ a composição $\varphi \circ \pi \in \mathcal{O}(U) \subset \mathcal{O}\left(U^{\prime}\right)$ define $\widetilde{C}$ localmente, já que $\left.\pi\right|_{U^{\prime}}$ é isomorfismo sobre a imagem $V^{\prime}=V \backslash\{P\}$.

Se $f \in \mathcal{O}\left(V^{\prime}\right)$, considere $f=a \cdot \varphi^{i}$ de forma que $\varphi \nmid a$. Então $f \circ \pi=$ $(a \circ \pi) \cdot(\varphi \circ \pi)^{i}$ e se por absurdo $a \circ \pi=(\varphi \circ \pi) \cdot g \operatorname{com} g \in \mathcal{O}\left(U^{\prime}\right)$, então 
$a=\varphi \cdot\left(g \circ \pi^{-1}\right) \operatorname{com} g \circ \pi^{-1} \in \mathcal{O}\left(V^{\prime}\right)$, uma contradição. Portanto

$$
\operatorname{ord}_{\widetilde{C}}(f \circ \pi)=i=\operatorname{ord}_{C}(f) \text {. }
$$

No caso geral, $f=g / h$ com $g, h \in \mathcal{O}(V)$ e o mesmo segue do caso anterior.

Em particular, isso mostra que para um divisor de $S$ qualquer $D=$ $a_{1} C_{1}+\ldots+a_{m} C_{m}$ seu pullback tem $a_{1} \widetilde{C_{1}}+\ldots+a_{m} \widetilde{C_{m}}$ como parte de seus fatores. O único coeficiente que não é claro é o do divisor excepcional $\mathcal{E}$. Em outras palavras,

$$
\pi^{*} D=a_{1} \widetilde{C_{1}}+\ldots+a_{n} \widetilde{C_{n}}+m \mathcal{E}
$$

onde $m$ é um número inteiro a princípio desconhecido. Podemos provar que $m$ é exatamente a soma das multiplicidades do ponto explodido $P$ em cada curva $C_{j}$. Para isso, basta provar o caso particular em que $D$ é um divisor primo.

Definição 5.9. Seja $C \subset \mathbb{A}^{2}$ uma curva plana afim definida pelo polinômio $f \in k\left[x_{1}, x_{2}\right]$, isto é, $C=\mathcal{Z}(f)$. Considere a decomposição de $f$ em termos homogêneos $f=F_{0}+F_{1}+\ldots+F_{\text {degf }}$ com $F_{i} \in k\left[x_{1}, x_{2}\right]_{i}$. A multiplicidade de $(0,0)$ na curva $C$ é o inteiro

$$
\operatorname{mult}_{(0,0)} C=\min \left\{i \in \mathbb{Z} \mid F_{i} \neq 0\right\} .
$$

Note que $\operatorname{mult}_{(0,0)} C \geq 1$ se e só se $(0,0) \in C$. A Definição 5.9 pode ser generalizada de modo a considerar $C \in S$ uma curva projetiva em uma superfície não-singular $S$ e $P \in S$.

Definição 5.10. Seja $\varphi: U \rightarrow V$ um isomorfismo de uma vizinhança aberta de $P$ em $S$ para uma vizinhança aberta de $(0,0)$ em $\mathbb{A}^{2}$ tal que $P \mapsto(0,0)$. Então definimos a multiplicidade de $P$ na curva $C$ como

$$
\text { mult }_{P} C=\operatorname{mult}_{(0,0)} \varphi(C)
$$

Proposição 5.3. Seja $\pi: \mathbb{P}_{P}^{2} \rightarrow \mathbb{P}^{2}$ o blow up de $\mathbb{P}^{2}$ no ponto $P$ e $C \subset \mathbb{P}^{2}$ uma curva com $m=$ mult $_{P} C$. Então $\pi^{*} C=\widetilde{C}+m \mathcal{E}$.

Prova. Vamos supor sem perda que $P=(1: 0: 0)$. Seja $F \in k[x, y, z]^{h}$ um polinômio homogêneo tal que $C=\mathcal{Z}(F)$, ou seja, $\left.C\right|_{\mathcal{U}_{0}}=\left.\operatorname{div}\left(F / x^{d}\right)\right|_{\mathcal{U}_{0}}$, onde $d=$ $\operatorname{deg} F$. Em $\mathcal{U}_{0}$, a função racional $F / x^{d}$ se comporta como a desomogeneização $f \in k[y, z]$ de $F$, definida por $f(y, z)=F(1, y, z)$. Temos que $f=F_{m}+F_{m+1}+$ $\ldots+F_{d}$ onde cada $F_{i} \in k[y, z]^{h}$ é homogêneo de grau $i$. 
Lembre que

$$
\pi^{-1}\left(\mathcal{U}_{0}\right) \cong Z(y t-z s) \subset \mathbb{A}_{(y, z)}^{2} \times \mathbb{P}_{(s: t)}^{1}
$$

e que

$$
T=\pi^{-1}\left(\mathcal{U}_{0}\right) \backslash \mathcal{Z}(s) \cong \mathcal{Z}(y t-z) \subset \mathbb{A}_{(y, z, t)}^{3}
$$

de forma que $T \cong \mathbb{A}_{(y, t)}^{2}$. Nesse aberto, a restrição $\left.\pi\right|_{T}: T \rightarrow \mathcal{U}_{0}$ é tal que $(y, t) \mapsto(y, y t)$ de forma que $\left.\mathcal{E}\right|_{T}=\mathcal{Z}(y) \cap T$ e

$$
\left.\pi^{*} f\right|_{T}=y^{m}\left(F_{m}(1, t)+y F_{m+1}(1, t)+\ldots+y^{d-m} F_{d}(1, t)\right) .
$$

Portanto $\operatorname{ord}_{\mathcal{E}}\left(\pi^{*} f\right)=m$, e então $\pi^{*} C \sim \widetilde{C}+m \mathcal{E}$.

Exemplo 33. Seja $\pi: \mathbb{P}_{P}^{2} \rightarrow \mathbb{P}^{2}$ o blowup de $\mathbb{P}^{2}$ no ponto $P$. O pullback de uma reta $L \in \operatorname{Div}\left(\mathbb{P}^{2}\right)$ que não passa por $P$ é simplesmente $\pi^{*} L=\widetilde{L}$ sua transformada estrita. Já se $L^{\prime} \in \operatorname{Div}\left(\mathbb{P}^{2}\right)$ é uma reta que passa por $P$, seu pullback é $\pi^{*} L^{\prime}=\widetilde{L^{\prime}}+\mathcal{E}$ sua transformada estrita mais o divisor excepcional do blowup. Isso significa que $\widetilde{L} \sim \widetilde{L}^{\prime}+\mathcal{E}$ como divisores de $\mathbb{P}_{P}^{2}$.
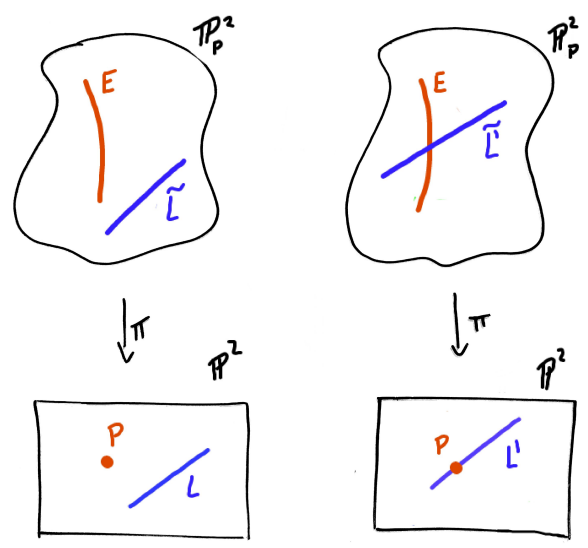

A Proposição 5.3 pode ser generalizada para o blowup de qualquer superfície não-singular.

Proposição 5.4. Seja $\pi: S_{P} \rightarrow S$ o blowup de uma superfície não-singular $S$ em $P \in S$ e $C \subset S$ uma curva com $m=$ mult $_{P} C$. Então $\pi^{*} C=\widetilde{C}+m \mathcal{E}$.

A demonstração da Proposição 5.4 se encontra na referência (HARTSHORNE, 1977) Capítulo 5, Proposição 3.6. Como consequência, podemos obter o seguinte:

Proposição 5.5. Seja $\pi: S_{P} \rightarrow S$ o blowup de uma superfície não-singular $S$ em $P \in S$. Então o pullback $\pi^{*}: \operatorname{Div}(S) \rightarrow \operatorname{Div}\left(S_{P}\right)$ é injetivo. 
Prova. Suponha que $D, D^{\prime} \in \operatorname{Div}(S)$ são tais que $\pi^{*} D=\pi^{*} D^{\prime}$, então $\widetilde{D}+m \mathcal{E}=$ $\widetilde{D^{\prime}}+m^{\prime} \mathcal{E}$ onde $m$ e $m^{\prime}$ são as respectivas multiplicidades de $D$ e $D^{\prime}$ em $P$. Como $\widetilde{D}$ e $\widetilde{D^{\prime}}$ não possuem o fator $\mathcal{E}$, teremos que $m=m^{\prime}$ e $\widetilde{D}=\widetilde{D^{\prime}}, \operatorname{logo} D=D^{\prime}$.

Perceba que a transformada estrita e o pullback coincidem no complementar do divisor excepcional $\mathcal{E}$. Isso significa que o pullback $\operatorname{Div}(S) \hookrightarrow$ $\operatorname{Div}\left(S_{P}\right)$ também induz um isomorfismo $\operatorname{Div}\left(S_{P}\right) \cong \operatorname{Div}(S) \oplus \mathbb{Z} \mathcal{E}$, que é diferente do encontrado no exemplo 31 , dado pela transformada estrita.

Esse segundo isomorfismo nos será mais interessante, pois a transformada estrita não respeita equivalência linear. Isso pode ser visto no exemplo anterior: $\widetilde{L} \nsim \widetilde{L^{\prime}}$. Mas já que o pullback preserva equivalência linear, podemos usar o pullback $\operatorname{Pic}(S) \rightarrow \operatorname{Pic}\left(S_{P}\right)$ para obter um homomorfismo $\operatorname{Pic}(S) \oplus \mathbb{Z}[\mathcal{E}] \rightarrow$ $\operatorname{Pic}\left(S_{P}\right)$ que leva qualquer divisor de $S$ em seu pullback e leva $\mathcal{E}$ em sua classe.

Nosso objetivo é verificar que este homomorfismo é um isomorfismo. $\operatorname{Como} \operatorname{Div}\left(S_{P}\right) \cong \operatorname{Div}(S) \oplus \mathbb{Z} \mathcal{E}$ usando o pullback, é claro que o homomorfismo $\operatorname{Pic}(S) \oplus \mathbb{Z}[\mathcal{E}] \rightarrow \operatorname{Pic}\left(S_{P}\right)$ acima é sobrejetivo. Em outras palavras, $\operatorname{Pic}\left(S_{P}\right)$ é gerado por $\mathcal{E}$ e os pullback de geradores de $\operatorname{Pic}(S)$, ainda que possam ter alguma relação. Então basta mostrar injetividade.

Para isso, antes mostraremos $\pi^{*}: \operatorname{Pic}(S) \rightarrow \operatorname{Pic}\left(S_{P}\right)$ é injetivo, isto é, se $D, D^{\prime}$ são divisores de $S$ tais que $\pi^{*} D \sim \pi^{*} D^{\prime}$ como divisores de $S_{P}$, então $D \sim D^{\prime}$. Basicamente a recíproca para a Proposição 5.1 no caso particular de explosões de superfícies.

Proposição 5.6. Seja $\pi: \mathbb{P}_{P}^{2} \rightarrow \mathbb{P}^{2}$ o blowup de $\mathbb{P}^{2}$ no ponto $P$. Então o pullback $\pi^{*}: \operatorname{Pic}\left(\mathbb{P}^{2}\right) \rightarrow \operatorname{Pic}\left(\mathbb{P}_{P}^{2}\right)$ é injetivo.

Prova. Como sabemos, $\operatorname{Pic}\left(\mathbb{P}^{2}\right) \cong \mathbb{Z}[L]$ onde $L$ é uma reta fixa em $\mathbb{P}^{2}$ qualquer. Podemos supor que $L$ não passa pelo ponto $P$, e então qualquer divisor $D \sim n L$ de $\mathbb{P}^{2}$ tem seu pullback $\pi^{*} D \sim n \widetilde{L}$. Desta forma, provar que $\pi^{*}: \operatorname{Pic}\left(\mathbb{P}^{2}\right) \rightarrow \operatorname{Pic}\left(\mathbb{P}_{P}^{2}\right)$ é injetivo é equivalente a provar que $n \widetilde{L}$ não é um divisor principal de $\mathbb{P}_{P}^{2}$ para nenhum $n \neq 0$.

De fato, suponha que $n \widetilde{L}$ é principal, então temos uma função racional $f \in K\left(\mathbb{P}_{P}^{2}\right)$ tal que $n \widetilde{L}=\operatorname{div}(f)$. Seja $U$ um aberto tal que $f \in \mathcal{O}(U)$ e suponha sem perda que $U \cap \mathcal{E}=\varnothing$. Ou seja, $\left.\pi\right|_{U}$ é um isomorfismo sobre sua imagem $V \subset \mathbb{P}^{2}$. Então $g=f \circ \pi^{-1} \in \mathcal{O}(V)$ satisfaz $\operatorname{ord}_{\widetilde{C}}(f)=\operatorname{ord}_{C}(g)$ para toda curva irredutível $C \subset \mathbb{P}^{2}$. Em particular, $\operatorname{div}(g)=n L$, portanto $n=0$.

Mais geralmente, temos os seguinte resultado, que pode ser encontrado em (HARTSHORNE, 1977) Capítulo 5, Proposição 3.2:

Proposição 5.7. Seja $\pi: S_{P} \rightarrow S$ é o blowup de $S$ em $P$, então o pullback $\pi^{*}: \operatorname{Pic}(S) \rightarrow \operatorname{Pic}\left(S_{P}\right)$ é injetivo. Mais geralmente, seja $\pi: S^{\prime} \rightarrow S$ um morfismo birracional, o pullback $\pi^{*}: \operatorname{Pic}(S) \rightarrow \operatorname{Pic}\left(S^{\prime}\right)$ é injetivo. 


\section{3}

\section{A forma de interseção}

Teorema 5.3 (Forma de interseção). Seja $S$ uma superfície projetiva nãosingular. Existe uma única forma bilinear simétrica $\operatorname{Div}(S) \times \operatorname{Div}(S) \rightarrow \mathbb{Z}$ tal que:

- se $C, C^{\prime} \in \operatorname{Div}(S)^{p}$ são duas curvas irredutiveis em $S$ distintas, então $C \cdot C^{\prime}$ é a quantidade de pontos da interseção $C \cap C^{\prime}$ contados com multiplicidade;

- dados $D, D^{\prime} \in \operatorname{Div}(S)$, o produto $D \cdot D^{\prime}$ só depende das classes de equivalência linear de $D$ e $D^{\prime}$, ou seja, passa ao quociente $\operatorname{Pic}(S) \times$ $\operatorname{Pic}(S) \rightarrow \mathbb{Z}$

Como referência, confira (HARTSHORNE, 1977) Capítulo V, Seção 1.1. Exemplo 34. Vamos analisar quando $S=\mathbb{P}^{2}$. Sejam $L$ e $L^{\prime}$ duas retas distintas em $\mathbb{P}^{2}$. Precisamos que $L \cdot L^{\prime}=1$, para satisfazer a primeira condição. Por outro lado, $L \sim L^{\prime}$, e portanto $L \cdot L=1$ pela segunda condição. Como $\operatorname{Pic}\left(\mathbb{P}^{2}\right) \cong \mathbb{Z} \cdot[L]$, a condição $L^{2}=1$ define uma única forma bilinear simétrica em $\operatorname{Div}\left(\mathbb{P}^{2}\right)$, a saber $D \cdot D^{\prime}=\operatorname{deg}(D) \cdot \operatorname{deg}\left(D^{\prime}\right)$.

Exemplo 35. Vamos analisar quando $S=\mathbb{P}_{P}^{2}$. Sabemos que $\operatorname{Pic}\left(\mathbb{P}_{P}^{2}\right)$ é gerado pelos elementos $\mathcal{L}$ e $\mathcal{E}$, onde $\mathcal{L}$ é o pullback de uma reta $L$ qualquer em $\mathbb{P}^{2}$ (que podemos supor que não passa por $P$ ) e $\mathcal{E}=\pi^{-1}(P)$. Para definir uma forma bilinear simétrica em $\operatorname{Pic}\left(\mathbb{P}_{P}^{2}\right)$, basta conhecermos $\mathcal{L}^{2}, \mathcal{L} \cdot \mathcal{E}$ e $\mathcal{E}^{2}$.

Com um argumento análogo ao do exemplo anterior, podemos concluir que a forma de interseção satisfaz $\mathcal{L}^{2}=1$, basta tomar o pullback de uma outra reta em $\mathbb{P}^{2}$ que também não passe por $P$.

Seja $\widetilde{L^{\prime}}$ a transformada estrita de uma reta $L^{\prime} \subset \mathbb{P}^{2}$ passando por $P$, sabemos que o pullback desta mesma reta por $\pi$ é $\pi^{*} L^{\prime}=\widetilde{L^{\prime}}+\mathcal{E} \sim \mathcal{L}$. Então $\mathcal{L} \cdot\left(\widetilde{L^{\prime}}+\mathcal{E}\right)=1$, e, por linearidade, $\mathcal{L} \cdot \mathcal{E}=1-\mathcal{L} \cdot \widetilde{L^{\prime}}=0$, pois $\mathcal{L}$ e $\widetilde{L^{\prime}}$ se intersectam em um único ponto com multiplicidade 1 , a pré-imagem pelo morfismo $\pi$ da interseção $L \cap L^{\prime}$.

Finalmente, usando mais uma vez que $\mathcal{L} \sim \widetilde{L^{\prime}}+\mathcal{E}$ temos que $\left(\widetilde{L^{\prime}}+\mathcal{E}\right) \cdot \mathcal{E}=0$, $\operatorname{logo} \mathcal{E}^{2}=-\widetilde{L^{\prime}} \cdot \mathcal{E}=-1$, pois $\widetilde{L^{\prime}}$ e $\mathcal{E}$ se intersectam em exatamente 1 ponto com multiplicidade 1 .

Podemos concluir que a forma de interseção é definida pelas equações $\mathcal{L}^{2}=1, \mathcal{L} \cdot \mathcal{E}=0$ e $\mathcal{E}^{2}=-1$. Repare que isso é suficiente para concluirmos que $[\mathcal{L}]$ e $[\mathcal{E}]$ não possuem relação como geradores de $\operatorname{Pic}\left(\mathbb{P}_{P}^{2}\right)$, pois se $n \mathcal{E} \sim m \mathcal{L}$, então $-n=n \mathcal{E} \cdot \mathcal{E}=m \mathcal{L} \cdot \mathcal{E}=0$ e também $m=0$. Com isso, concluímos que $\operatorname{Pic}\left(\mathbb{P}_{P}^{2}\right) \cong \mathbb{Z}[\mathcal{L}] \oplus \mathbb{Z}[\mathcal{E}]$. 
Note que apesar de $C \cdot C^{\prime}$ ser não negativo sempre que $C$ e $C^{\prime}$ são curvas irredutíveis distintas, $C^{2}$ não precisa ser. De fato, encontramos uma curva em $\mathbb{P}_{P}^{2}$ cujo produto com si mesma é -1 , a saber, o divisor excepcional $\mathcal{E}$.

Definição 5.11. Dada uma superfície não-singular $S$, dizemos que uma curva $C \subset S$ é uma curva -1 se $C \cong \mathbb{P}^{1}$ e $C \cdot C=-1$.

Exemplo 36. Seja $S=\mathbb{P}_{P_{0} P_{1}}^{2}$ com $P_{0}, P_{1} \in \mathbb{P}^{2}$ distintos, $\pi: S \rightarrow \mathbb{P}^{2}$ a composição das explosões $\pi_{0}: \mathbb{P}_{P_{0}}^{2} \rightarrow \mathbb{P}^{2}$ e $\pi_{1}: \mathbb{P}_{P_{0} P_{1}}^{2} \rightarrow \mathbb{P}_{P_{0}}^{2}$ e $\mathcal{E}_{i}=\pi^{-1}\left(P_{i}\right), i=0,1$. Nesse caso, devido à Proposição 5.4 no blowup $\pi_{1}$, é claro que $\mathcal{E}_{0}$ é o pullback por $\pi_{1}$ do divisor excepcional $E_{0}=\pi_{0}^{-1}\left(P_{0}\right)$ do blowup $\pi_{0}$.

Podemos concluir indutivamente que $\operatorname{Pic}(S)=\mathbb{Z}[\mathcal{L}] \oplus \mathbb{Z}\left[\mathcal{E}_{0}\right] \oplus \mathbb{Z}\left[\mathcal{E}_{1}\right]$, onde $\mathcal{L}$ é o pullback por $\pi$ de uma reta $L$ qualquer em $\mathbb{P}^{2}$. De forma análoga ao exemplo anterior, teremos que $\mathcal{E}_{i}^{2}=-1$ para $i=0,1$, e como são disjuntos, $\mathcal{E}_{0} \cdot \mathcal{E}_{1}=0$. Além disso, $\mathcal{L}^{2}=1$ e $\mathcal{L} \cdot \mathcal{E}_{i}=0, i=0,1$.

Agora considere a reta $L_{01}$ que liga os pontos $P_{0}$ e $P_{1}$ em $\mathbb{P}^{2}$. Seu pullback por $\pi$ é $\widetilde{L_{01}}+\mathcal{E}_{0}+\mathcal{E}_{1}$. Ou seja, podemos inferir que $\widetilde{L_{01}} \sim \mathcal{L}-\mathcal{E}_{0}-\mathcal{E}_{1}$, portanto ${\widetilde{L_{01}}}^{2}=\left(\mathcal{L}-\mathcal{E}_{0}-\mathcal{E}_{1}\right)^{2}=1-1-1=-1$. Concluímos então que $\widetilde{L_{01}}$ é uma curva -1 .

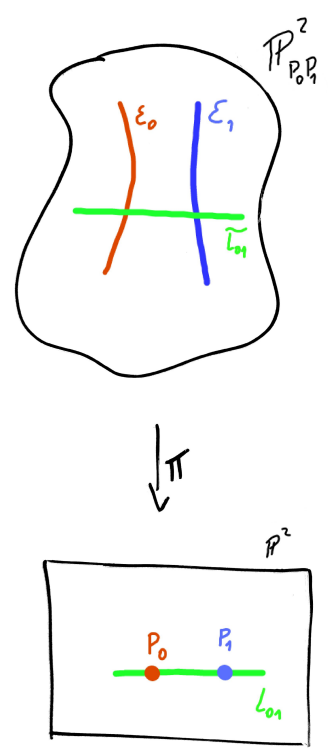

Antes de ingressar na próxima Proposição, vale observar que isomorfismos preservam a forma de interseção em superfícies. Mais precisamente, se $\mu: S \rightarrow$ $S^{\prime}$ é um isomorfismo, e $C_{1}, C_{2} \subset S$ são duas curvas, então $C_{1} \cdot C_{2}=\mu\left(C_{1}\right) \cdot \mu\left(C_{2}\right)$, onde o produto da esquerda é dado pela forma de interseção em $S$ e o da direita pela forma de interseção em $S^{\prime}$. Mais ainda, podemos considerar o isomorfismo de grupos $\bar{\mu}: \operatorname{Div}(S) \rightarrow \operatorname{Div}\left(S^{\prime}\right)$ induzido por $\mu$ de forma linear, e teremos que dados $D_{1}, D_{2} \in \operatorname{Div}(S)$ dois divisores, então $D_{1} \cdot D_{2}=\bar{\mu}\left(D_{1}\right) \cdot \bar{\mu}\left(D_{2}\right)$. 
Proposição 5.8 (Moving Lemma). Seja $D \in \operatorname{Div}(X)$ um divisor de uma variedade não-singular $X$, e $P_{1}, \ldots, P_{m} \in X$ finitos pontos. Então existe um divisor $D^{\prime}$ tal que $D^{\prime} \sim D$ e $P_{i} \notin \operatorname{Supp}\left(D^{\prime}\right)$ para todo $i=1, \ldots, m$.

Prova. Teorema 1, Capítulo 3 Seção 1.3 (SHAFAREVICH, 1994).

Proposição 5.9. O pullback preserva a forma de interseção. Mais precisamente, se $\pi: S^{\prime} \rightarrow S$ é um morfismo birracional de superfícies lisas com $D$, $D^{\prime} \in \operatorname{Div}(S)$, então $D \cdot D^{\prime}=\pi^{*} D \cdot \pi^{*} D^{\prime}$.

Prova. Novamente, pelo Teorema 4.1 basta provar o caso em que $S^{\prime}=S_{P}$ é a explosão de $S$ em um certo ponto $P$. Nesse caso, a Proposição é óbvia no caso especial em que $D$ e $D^{\prime}$ são curvas irredutíveis distintas que não passam por $P$, afinal vão estar em isomorfismo com seus pullbacks e vão preservar a quantidade de interseções com multiplicidade.

No caso geral em que $D$ e $D^{\prime}$ são divisores quaisquer de $S$, podemos supor que $P$ não pertence ao suporte de $D$, caso contrário basta substituí-lo sem perdas por um divisor linearmente equivalente usando o Moving Lemma (Proposição 5.8). Agora fixe um ponto em cada divisor primo que componha a soma de $D$ e mova $D^{\prime}$ se necessário para obter um outro divisor de $S$ que seja linearmente equivalente, dessa vez evitando não só $P$ mas também todos esses outros pontos fixados. Portanto agora temos $D \sim a_{1} C_{1}+\ldots+a_{m} C_{m}$ e $D^{\prime} \sim a_{m+1} C_{m+1}+\ldots+a_{m+n} C_{m+n} \operatorname{com} C_{i} \neq C_{j}$ sempre que $i \neq j$ e a Proposição deriva do caso especial.

Teorema 5.4 (Contratibilidade de Castelnuovo). Seja $S^{\prime}$ uma superfície nãosingular e $C \subset S^{\prime}$ uma curva -1. Então existe uma superfície não-singular $S$ e um ponto $P \in S$ com blowup $\pi: S_{P} \rightarrow S$ tal que $S^{\prime} \cong S_{P} e C \cong \pi^{-1}(P)$ por esse isomorfismo. Dizemos que $S$ é a contração de $C$.

Prova. Teorema 5.7 do Capítulo V de (HARTSHORNE, 1977)

Exemplo 37. Em $S^{\prime}=\mathbb{P}_{P_{0} P_{1}}^{2}$, com $P_{0}, P_{1} \in \mathbb{P}^{2}$ distintos, $\widetilde{L_{01}}$ é uma curva -1 . A contração dessa curva é isomorfa a $\mathbb{P}^{1} \times \mathbb{P}^{1}$. 


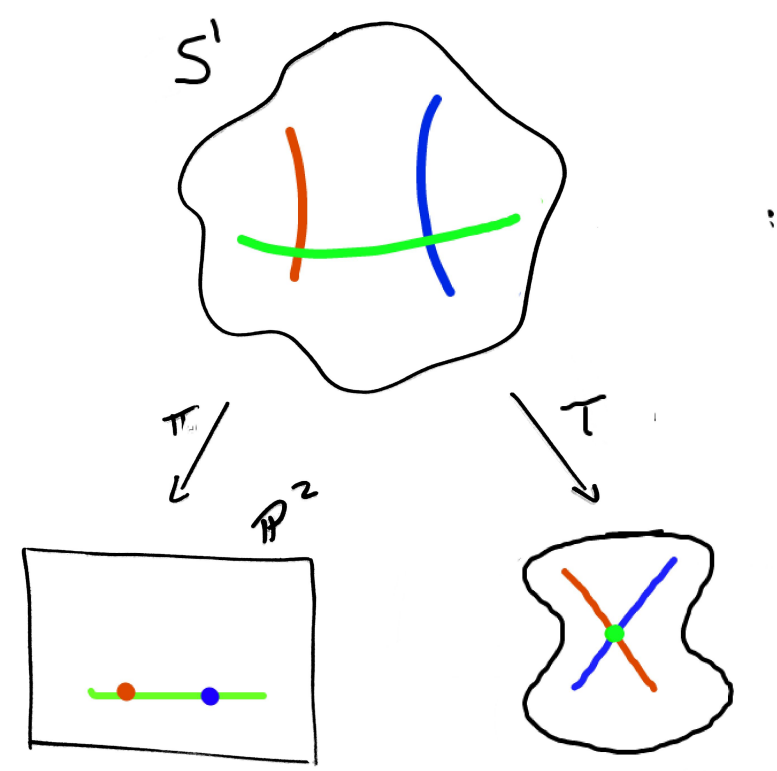

Exemplo 38. Em $S^{\prime}=\mathbb{P}_{P_{0} P_{1} P_{2}}^{2}$, com $P_{0}=(1: 0: 0), P_{1}=(0: 1: 0), P_{2}=(0: 0: 1) \epsilon$ $\mathbb{P}^{2}$, as curvas $\widetilde{L_{i j}}$ são -1 , por motivos análogos, e seja $\pi^{\prime}: S^{\prime} \rightarrow \mathbb{P}^{2}$ a composição das três explosões.

Podemos contrair uma curva por vez e ainda garantir que as outras continuam sendo curvas -1. Isso porque, por serem disjuntas, contraindo apenas uma, temos um blowup cujo divisor excepcional não intersecta as outras, ou seja, elas serão o pullback de suas imagens, e o pullback preserva a forma de interseção.

Ao usar o Teorema de Contratibilidade três vezes, teremos uma superfície $S$ e três pontos $Q_{0}, Q_{1}, Q_{2}$ (a princípio não necessariamente todos em $S$, mas em explosões de $S$ ) e a composição de três explosões $\pi: S_{Q_{0} Q_{1} Q_{2}} \rightarrow S$, de forma que $S^{\prime} \stackrel{\sim}{\rightarrow} S_{Q_{0} Q_{1} Q_{2}}$ mapeando isomorficamente $\widetilde{L_{i j}}$ em $\pi^{-1}\left(Q_{k}\right)$ onde $\{i, j, k\}=\{0,1,2\}$. Porém, como as três curvas contraídas são disjuntas, fica claro que a explosão ocorre em três pontos distintos de $S$, isto é, $Q_{0}, Q_{1}, Q_{2} \in S$.

Ocorre que teremos $S=\mathbb{P}^{2}$ e cada $Q_{i}=P_{i}$. De fato, relembre dos exemplos 21 e 22 em que estudamos o morfismo $\widetilde{\Phi}=\Phi \circ \pi$ que resolve as indeterminações da Transformação de Cremona $\Phi$, onde $\pi: \mathbb{P}_{P_{0} P_{1} P_{2}}^{2} \rightarrow \mathbb{P}^{2}$ é a composição das três explosões. Vimos que $\widetilde{\Phi}$ é por si só a composição de três explosões de $\mathbb{P}^{2}$ nos pontos $P_{0}, P_{1}, P_{2}$, cujos divisores excepcionais são respectivamente as 


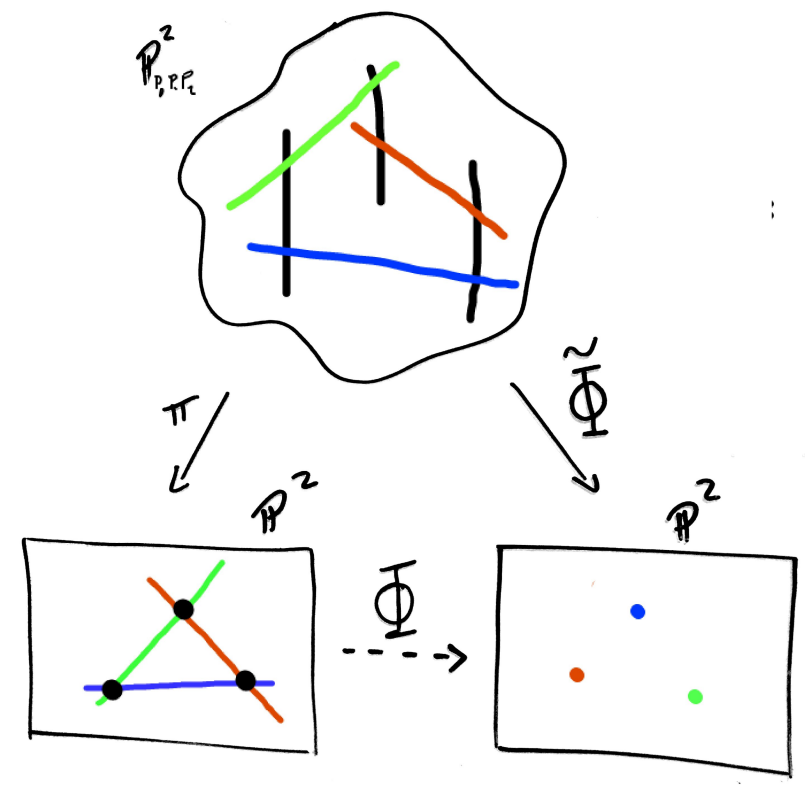

transformadas estritas $\widetilde{L_{12}}, \widetilde{L_{02}}, \widetilde{L_{01}}$ das retas $L_{i j} \subset \mathbb{P}^{2}$ por $\pi$.

Em termos do Teorema de Contratibilidade, teremos que existe um isomorfismo $\widetilde{\widetilde{\Phi}}: \mathbb{P}_{P_{0} P_{1} P_{2}}^{2} \rightarrow \mathbb{P}_{P_{0} P_{1} P_{2}}^{2}$ que leva cada curva $\widetilde{L_{i j}}$ isomorficamente em $\mathcal{E}_{k}$, onde $\{i, j, k\}=\{0,1,2\}$. Teremos em particular que $\pi \circ \widetilde{\widetilde{\Phi}}=\widetilde{\Phi}=\Phi \circ \pi$.

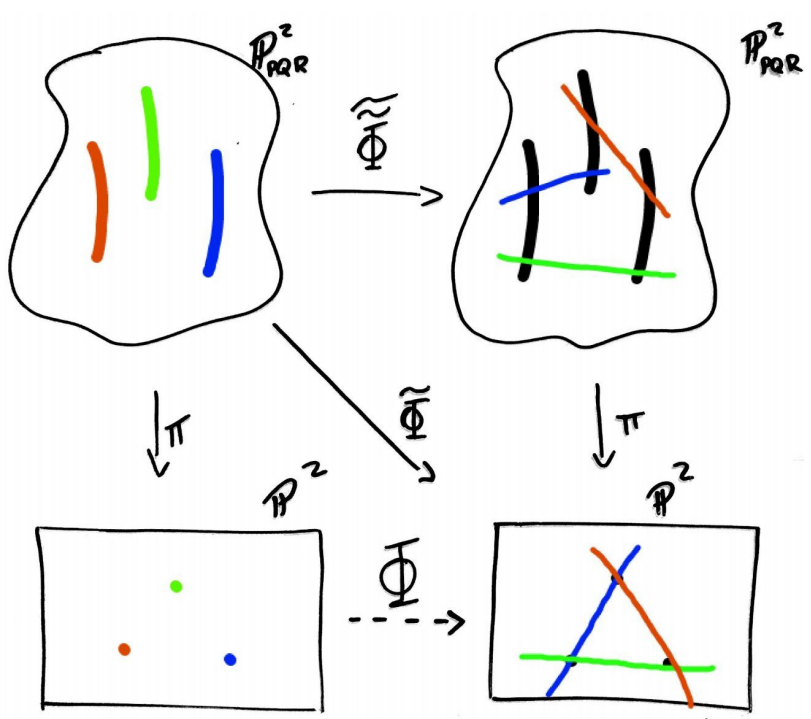




\section{6 \\ Interações com Geometria Hiperbólica}

\section{1}

\section{Motivação}

Consideraremos agora $\pi: S \rightarrow \mathbb{P}^{2}$ a composição dos blowups $S=$ $\mathbb{P}_{P_{1} \ldots P_{n}}^{2} \rightarrow \ldots \rightarrow \mathbb{P}_{P_{1} P_{2}}^{2} \rightarrow \mathbb{P}_{P_{1}}^{2} \rightarrow \mathbb{P}^{2}$ de $\mathbb{P}^{2}$ em $n$ pontos (não necessariamente todos em $\mathbb{P}^{2}$ ) e a forma de interseção definida para os divisores de $S$.

Lembre que

$$
\operatorname{Pic}(S) \cong \mathbb{Z}[\mathcal{L}] \oplus \mathbb{Z}\left[\mathcal{E}_{1}\right] \oplus \ldots \oplus \mathbb{Z}\left[\mathcal{E}_{n}\right]
$$

por indução, onde $\mathcal{L}$ é o pullback de uma reta qualquer em $\mathbb{P}^{2}$ e para cada $i=1, \ldots, n, \mathcal{E}_{i}$ é pullback final do divisor excepcional do blowup no ponto $P_{i}$. Mais precisamente, seja $E_{i} \subset \mathbb{P}_{P_{1} \ldots P_{i}}^{2}$ o divisor excepcional do blowup $\mathbb{P}_{P_{1} \ldots P_{i}}^{2} \rightarrow \mathbb{P}_{P_{1} \ldots P_{i-1}}^{2}$, então $\mathcal{E}_{i} \subset \mathbb{P}_{P_{1} \ldots P_{n}}^{2}$ é o pullback de $E_{i}$ pela composição $\mathbb{P}_{P_{1} \ldots P_{n}}^{2} \rightarrow \mathbb{P}_{P_{1} \ldots P_{i}}^{2}$

Aqui, a forma de interseção é dada por $\mathcal{L}^{2}=1, \mathcal{L} \cdot \mathcal{E}_{i}=0$ para todo $i=1, \ldots, n$ e $\mathcal{E}_{i} \cdot \mathcal{E}_{j}=-\delta_{i j}$ para todo $i, j=1, \ldots, n$. Desta forma, dados

$$
D \sim a_{0} \mathcal{L}+\sum_{i=1}^{n} a_{i} \mathcal{E}_{i}, D^{\prime} \sim b_{0} \mathcal{L}+\sum_{i=1}^{n} b_{i} \mathcal{E}_{i}
$$

dois divisores de $\mathbb{P}_{P_{1} \ldots P_{n}}^{2}$, então

$$
D \cdot D^{\prime}=a_{0} b_{0}-\sum_{i=1}^{n} a_{i} b_{i}
$$

Também podemos considerar o espaço vetorial $\operatorname{Div}(S)_{\mathbb{R}}$ dos $\mathbb{R}$-divisores de $S$ cujos elementos são

$$
D=\sum_{Z \in \operatorname{Div}(S)^{p}} x_{Z} Z
$$

com $x_{Z} \in \mathbb{R}$ para todo $Z \in \operatorname{Div}(S)^{p}$ e $x_{Z} \neq 0$ para finitos $Z \in \operatorname{Div}(S)^{p}$. Com a relação de equivalência $D \sim D^{\prime}$ se $D-D^{\prime}$ é uma combinação linear com coeficientes reais de divisores principais, temos também o espaço vetorial real

$$
\operatorname{Pic}(S)_{\mathbb{R}}=\frac{\operatorname{Div}(S)_{\mathbb{R}}}{\sim} \cong \mathbb{R}^{n+1}
$$

ou equivalentemente,

$$
\operatorname{Pic}(S)_{\mathbb{R}}=\operatorname{Pic}(S) \otimes_{\mathbb{Z}} \mathbb{R}
$$


Nesse espaço vetorial, de base $\left\{[\mathcal{L}],\left[\mathcal{E}_{1}\right], \ldots,\left[\mathcal{E}_{n}\right]\right\}$, a forma de assinatura $(1, n)$

$$
\left[x_{0} \mathcal{L}+x_{1} \mathcal{E}_{1}+\ldots+x_{n} \mathcal{E}_{n}\right] \cdot\left[y_{0} \mathcal{L}+y_{1} \mathcal{E}_{1}+\ldots+y_{n} \mathcal{E}_{n}\right]=x_{0} y_{0}-\sum_{i=1}^{n} x_{i} y_{i}
$$

generaliza a forma de interseção entre dois divisores com coeficientes inteiros.

O espaço vetorial $\mathbb{R}^{n+1}$ com a forma de assinatura $(1, n)$ é conhecido como o Espaço de Minkowski, onde é definido o modelo do hiperbolóide para o espaço hiperbólico de dimensão $n, \mathbb{H}^{n}$ (veja a Seção 6.2 a seguir). Então, se $f$ é um automorfismo de $S$, sabemos que $f$ induz um automosfismo de grupo de $\operatorname{Pic}(S)$ que preserva a forma de interseção, e se estende a um automorfismo linear em $\operatorname{Pic}(S)_{\mathbb{R}}$, que preserva a forma de assinatura $(1, n)$. Veremos que, por causa disso, $f$ induz uma isometria de $\mathbb{H}^{n}$, e assim definimos uma ação de $\operatorname{Aut}(S)$ em $\mathbb{H}^{n}$ por isometrias. Como isometrias são bastante estudadas em Geometria Hiperbólica, esse ponto de vista tem muito potencial para entendermos melhor o grupo de Cremona $\operatorname{Cr}\left(\mathbb{P}^{2}\right)$.

Exemplo 39. Considere $S=\mathbb{P}_{P_{0} P_{1} P_{2}}^{2}$ e seu automorfismo $f=\widetilde{\widetilde{\Phi}}: S \rightarrow S$ estudado no exemplo 38. Por ser um automorfismo de $S, f$ induz um automorfismo em $\operatorname{Div}(S)$ que preserva o subgrupo dos divisores principais (induzindo também um automorfismo em $\operatorname{Pic}(S))$ e também a forma de interseção.

Por exemplo, vimos que cada transformada estrita $\widetilde{L_{i j}}$ é levada isomorficamente em $\mathcal{E}_{k}$, com $\{i, j, k\}=\{0,1,2\}$. Como $\widetilde{\widetilde{\Phi}}=\pi^{-1} \circ \Phi \circ \pi$, onde $\pi: S \rightarrow \mathbb{P}^{2}$ é a composição dos blowups, então

$$
\widetilde{\widetilde{\Phi}} \widetilde{\widetilde{\Phi}}=\left(\pi^{-1} \circ \Phi \circ \pi\right) \circ\left(\pi^{-1} \circ \Phi \circ \pi\right)=\mathrm{Id}
$$

e consequentemente cada $\mathcal{E}_{i}$ é levado isomorficamente por $\widetilde{\widetilde{\Phi}}$ na transformada estrita $\widetilde{L_{j k}}, \operatorname{com}\{i, j, k\}=\{0,1,2\}$.

O próximo passo para entendermos melhor o automorfismo de $\operatorname{Div}(S)$ induzido por $\widetilde{\widetilde{\Phi}}$ é determinarmos a imagem do pullback de uma reta qualquer. Seja $L \subset \mathbb{P}^{2}$ uma reta em $\mathbb{P}^{2}$ que não passa por nenhum dos pontos coordenados. É fácil verificar que $\Phi$ leva $L$ isomorficamente em uma cônica $C \subset \mathbb{P}^{2}$ que passa pelos três pontos coordenados. Isso indica que $\pi^{*} L$ é levado isomorficamente em $\widetilde{C}$ por $\widetilde{\widetilde{\Phi}}$.

Como um automorfismo em

$$
\operatorname{Pic}(S) \cong \mathbb{Z}[\mathcal{L}] \oplus \mathbb{Z}\left[\mathcal{E}_{0}\right] \oplus \mathbb{Z}\left[\mathcal{E}_{1}\right] \oplus \mathbb{Z}\left[\mathcal{E}_{2}\right]
$$




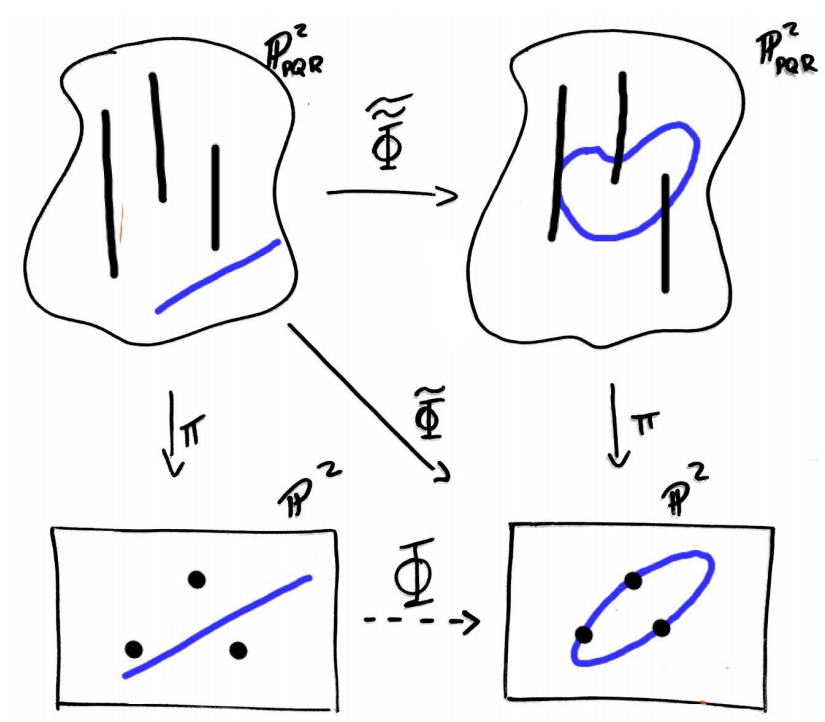

podemos verificar que

$$
\left[\mathcal{E}_{i}\right] \mapsto\left[\widetilde{L_{j k}}\right]=[\mathcal{L}]-\left[\mathcal{E}_{j}\right]-\left[\mathcal{E}_{k}\right]
$$

sempre que $\{i, j, k\}=\{0,1,2\}$, e que

$$
[\mathcal{L}] \mapsto[\widetilde{C}]=2[\mathcal{L}]-\left[\mathcal{E}_{0}\right]-\left[\mathcal{E}_{1}\right]-\left[\mathcal{E}_{2}\right]
$$

pois como a cônica $C$ passa por cada ponto coordenado com multiplicidade 1 , por ser não-singular, então

$$
\widetilde{C}=\pi^{*} C-\mathcal{E}_{0}-\mathcal{E}_{1}-\mathcal{E}_{2} \sim \pi^{*} 2 L-\mathcal{E}_{0}-\mathcal{E}_{1}-\mathcal{E}_{2} \sim 2 \pi^{*} L-\mathcal{E}_{0}-\mathcal{E}_{1}-\mathcal{E}_{2} .
$$

Esse automorfismo de $\operatorname{Pic}(S)$ se estende a um automorfismo linear de $\operatorname{Pic}(S)_{\mathbb{R}}$ dado pela matriz

$$
\left(\begin{array}{cccc}
2 & 1 & 1 & 1 \\
-1 & 0 & -1 & -1 \\
-1 & -1 & 0 & -1 \\
-1 & -1 & -1 & 0
\end{array}\right)
$$

Terminaremos esta motivação com o seguinte Teorema. Para referência, considere (DOLGACHEV; ISKOVSKIKH, 2009) Lema 3.5

Teorema 6.1. Qualquer subgrupo finito do Grupo de Cremona $\operatorname{Cr}\left(\mathbb{P}_{\mathbb{C}}^{2}\right)$ admite um levantamento a um subgrupo do grupo de automorfismos Aut $(S)$ de algum modelo $S$ de $\mathbb{P}^{2}$.

Aplicado ao nosso contexto, como corolário teremos que todo subgrupo 
finito de $\operatorname{Cr}\left(\mathbb{P}_{\mathbb{C}}^{2}\right)$ age por isometrias em um espaço hiperbólico de dimensão finita.

\section{2}

\section{O modelo do hiperbolóide para $\mathbb{H}^{n}$}

Como referência geral desta subseção usamos as notas de aula de Julien Paupert (PAUPERT, 2016).

$\operatorname{Em} \mathbb{R}^{n+1}$, considere a forma bilinear simétrica de assinatura $(1, n)$, isto é,

$$
\left(u_{0}, u_{1}, \ldots, u_{n}\right) \cdot\left(v_{0}, v_{1}, \ldots, v_{n}\right)=u_{0} v_{0}-\sum_{i=1}^{n} u_{i} v_{i},
$$

e defina a folha de hiperbolóide

$$
\mathbb{H}^{n}=\left\{v=\left(v_{0}, v_{1}, \ldots, v_{n}\right) \in \mathbb{R}^{n+1} \mid v \cdot v=1 \text { e } v_{0}>0\right\}
$$

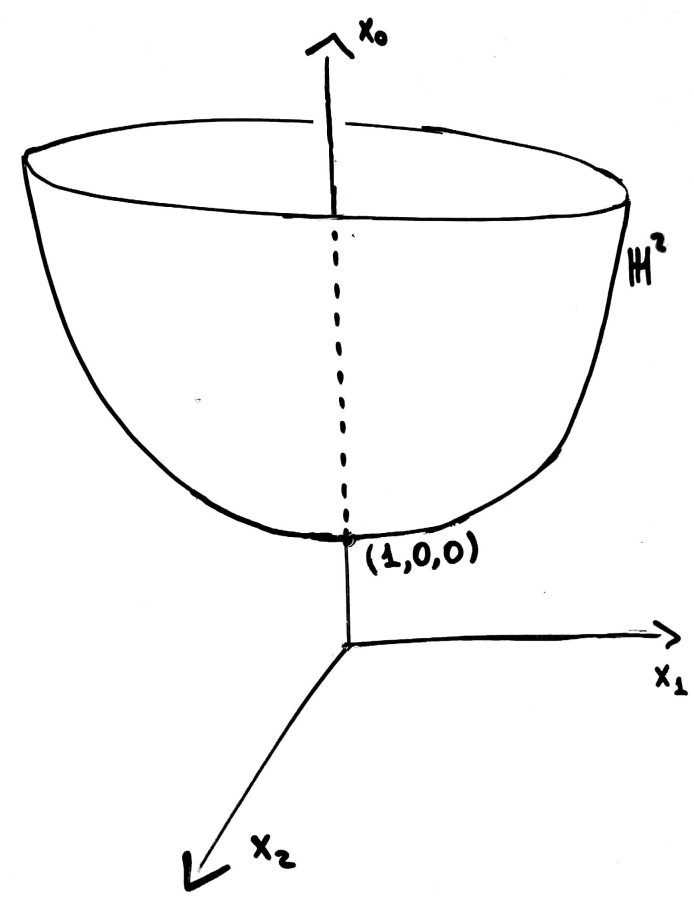

Proposição 6.1. O subconjunto $\mathbb{H}^{n} \subset \mathbb{R}^{n+1}$ é uma hipersuperfície suave, $e$ para todo $v \in \mathbb{H}^{n}$, o espaço tangente $T_{v} \mathbb{H}^{n}$ coincide com $v^{\perp}$.

Prova. De fato, a função $f: \mathbb{R}^{n+1} \rightarrow \mathbb{R}$ dada por $v \mapsto v \cdot v$ satisfaz $f(v+u)=$ $f(v)+2 v \cdot u+u \cdot u$, e podemos ver que $\mathrm{d}_{v} f(u)=2 v \cdot u$. Portanto $\mathrm{d}_{v} f$ tem 
posto 1 para todo $v \neq 0$ e 1 é valor regular de $f$, de forma que a pré-imagem $\mathbb{H}^{n}=f^{-1}(1)$ do valor 1 é uma variedade suave de codimensão 1. Em particular, temos que, para todo $v \in \mathbb{H}^{n}$, o espaço tangente $T_{v} \mathbb{H}^{n}=\operatorname{Nuc}\left(\mathrm{d}_{v} f\right)=v^{\perp}$.

Proposição 6.2. Em cada $T_{v} \mathbb{H}^{n}$ com $v \in \mathbb{H}^{n}$, a forma (6.1) é negativa definida.

Prova. Sejam $v=\left(v_{0}, v_{1}, \ldots, v_{n}\right) \in \mathbb{H}^{n}$ e $x=\left(x_{0}, x_{1}, \ldots, x_{n}\right) \in T_{v} \mathbb{H}^{n}$ tal que $x \neq 0$. Nosso objetivo é provar que $x \cdot x<0$.

Sabemos que $v \cdot v=1$ e que $x \cdot v=0$, ou seja,

$$
\sum_{i=1}^{n} v_{i}^{2}=v_{0}^{2}-1, \quad \sum_{i=1}^{n} x_{i} v_{i}=x_{0} v_{0}
$$

Portanto, pela desigualdade de Cauchy-Schwarz,

$$
x_{0}^{2} v_{0}^{2}<\left(v_{0}^{2}-1\right) \cdot\left(\sum_{i=1}^{n} x_{i}^{2}\right) .
$$

(Note que nunca ocorrerá igualdade de Cauchy-Schwarz, pois $x$ seria um múltiplo não nulo de $v$, ao mesmo tempo que $x \cdot v=0$ o que implicaria em $v \cdot v=0$, um absurdo.) Reagrupando a desigualdade, temos

$$
0 \leq \sum_{i=1}^{n} x_{i}^{2}<\left(\left(\sum_{i=1}^{n} x_{i}^{2}\right)-x_{0}^{2}\right) v_{0}^{2} .
$$

Como $v_{0}^{2} \geq 1$, temos que

$$
\left(\sum_{i=1}^{n} x_{i}^{2}\right)-x_{0}^{2}>0 \Rightarrow x \cdot x<0 .
$$

Em particular, dado $v \in \mathbb{H}^{n}$, o produto $T_{v} \mathbb{H}^{n} \times T_{v} \mathbb{H}^{n} \rightarrow \mathbb{R}$ definido por

$$
\langle x, y\rangle_{v}=-x \cdot y
$$

é um produto interno, que varia suavemente com $v$, e portanto induz uma métrica Riemanniana em $\mathbb{H}^{n}$ da seguinte forma. Se $\gamma:[0,1] \rightarrow \mathbb{H}^{n}$ é um caminho suave cuja integral

$$
\int_{0}^{1}\left\langle\gamma^{\prime}(t), \gamma^{\prime}(t)\right\rangle_{\gamma(t)} \mathrm{d} t
$$

está bem definida, definimos seu comprimento $l(\gamma)$ como a integral acima. A distância entre dois pontos $u, v \in \mathbb{H}^{n}$ é dada por $\mathrm{d}(u, v)=\inf \{l(\gamma) \mid \gamma(0)=$ $u, \gamma(1)=v\}$. Um caminho que realiza a distância entre dois pontos é dito um segmento de geodésica.

Teorema 6.2. A distância acima satisfaz $d(u, v)=\cosh ^{-1}(u \cdot v)$ e é atingida por um único segmento de geodésica, contido em $\mathbb{H}^{n} \cap \operatorname{Span}(u, v)$. 


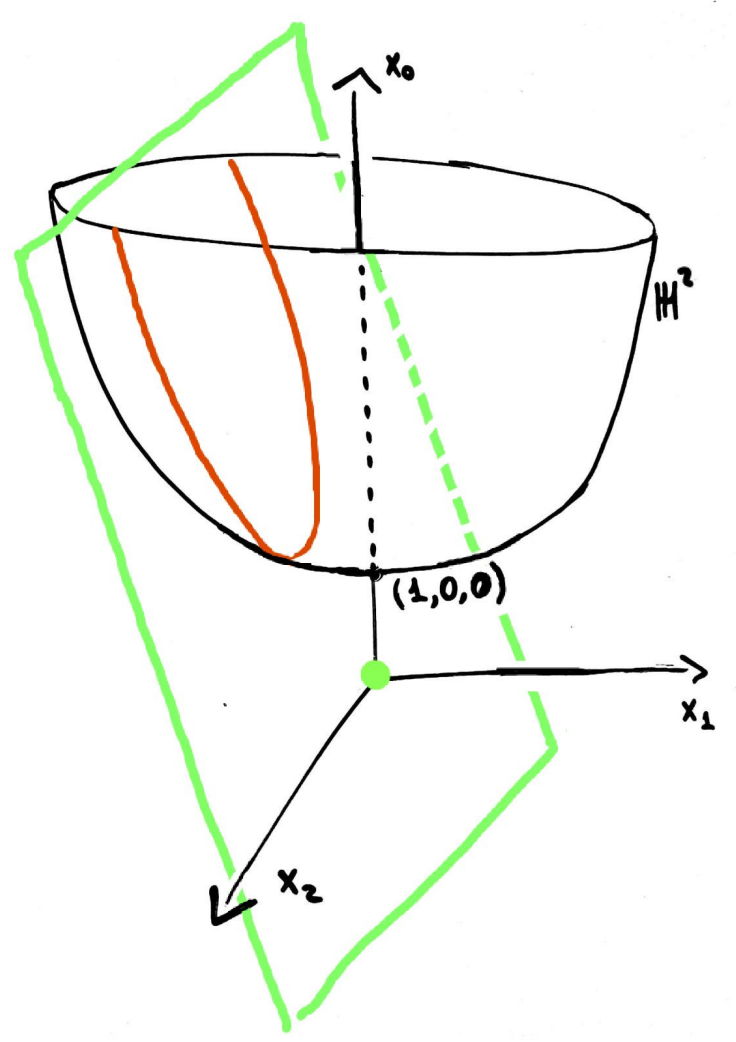

O espaço $\mathbb{H}^{n}$ munido do produto interno (6.2) em cada $T_{v} \mathbb{H}^{n}$ é uma variedade Riemanniana completa de dimensão $n$ e curvatura constante -1 . Tautologicamente, $\mathbb{H}^{n}$ é CAT(-1) (a definição de CAT(-1) se encontra na referência do Capítulo, (PAUPERT, 2016)), e também é $\delta$-hiperbólico, isto é, existe $\delta>0$ tal que, para todo triângulo geodésico em $\mathbb{H}^{n}$, cada lado está contido na $\delta$-vizinhança da união dos outros dois. As definições de CAT(-1) e de $\delta$-hiperbólico não são relevantes no nosso estudo, porém mais tarde vamos precisar dessas hipóteses no Teorema 8.2.

A projeção natural $\mathbb{R}^{n+1} \backslash\{0\} \rightarrow \mathbb{P}_{\mathbb{R}}^{n}$ induz uma bijeção entre $\mathbb{H}^{n}$ e sua imagem em $\mathbb{P}_{\mathbb{R}}^{n}$. Nesse sentido, a imagem do cone de luz $C=\left\{v \in \mathbb{R}^{n+1} \mid v \cdot v=0\right\}$ define o bordo $\partial \mathbb{H}^{n}$.

\section{3}

\section{Isometrias de $\mathbb{H}^{n}$}

Definição 6.1. Uma isometria Riemanniana (ou simplesmente uma isometria) de $\mathbb{H}^{n}$ é um difeomorfismo $f: \mathbb{H}^{n} \rightarrow \mathbb{H}^{n}$ que preserva o produto interno nos espaços tangentes, isto é, para todo $v \in \mathbb{H}^{n}$ e $x, y \in T_{v} \mathbb{H}^{n}$,

$$
\left\langle d_{v} f(x), d_{v} f(y)\right\rangle_{f(v)}=\langle x, y\rangle_{v}
$$


ou equivalentemente,

$$
d_{v} f(x) \cdot d_{v} f(y)=x \cdot y
$$

Considere o conjunto das transformações lineares em $\mathbb{R}^{n+1}$ que preservam a forma bilinear (6.1), isto é,

$$
O(1, n)=\left\{A \in \operatorname{GL}(n+1, \mathbb{R}) \mid A u \cdot A v=u \cdot v \forall u, v \in \mathbb{R}^{n+1}\right\}
$$

Observe que as duas componentes conexas $\mathbb{H}^{n} \mathrm{e}-\mathbb{H}^{n}$ do hiperbolóide definido pela equação $v \cdot v=1$ ou são invariantes ou são trocadas por um elemento $A \in O(1, n)$. Desta forma, considere o subgrupo dos elementos que preservam $\mathbb{H}^{n}$

$$
O^{+}(1, n)=\left\{A \in O(1, n) \mid A\left(\mathbb{H}^{n}\right)=\mathbb{H}^{n}\right\} .
$$

Teorema 6.3. As isometrias de $\mathbb{H}^{n}$ são exatamente as restrições $\left.A\right|_{\mathbb{H}^{n}}$ dos elementos $A \in O^{+}(1, n)$.

As isometrias de $\mathbb{H}^{n}$ são classificadas em três tipos: elíptica, parabólica ou hiperbólica, dependendo do seu deslocamento, definido a seguir.

Definição 6.2. Seja $f: \mathbb{H}^{n} \rightarrow \mathbb{H}^{n}$ uma isometria. Definimos então o deslocamento de $f$ como $L(f)=\inf \left\{d(v, f(v)) \mid v \in \mathbb{H}^{n}\right\}$.

Definição 6.3. Uma isometria $f$ de $\mathbb{H}^{n}$ é dita

- elíptica se $L(f)$ é atingido por algum $v \in \mathbb{H}^{n}$ e $L(f)=0$.

- parabólica se $L(f)$ não é atingido por nenhum $v \in \mathbb{H}^{n}$.

- hiperbólica se $L(f)$ é atingido por algum $v \in \mathbb{H}^{n}$ e $L(f)>0$.

Proposição 6.3. Se $f$ é parabólica, então $L(f)=0$.

Também podemos decidir se $f$ é elíptica, parabólica ou hiperbólica dependendo da quantidade de pontos fixos em $\mathbb{H}^{n}$ e em $\partial \mathbb{H}^{n}$. Mais precisamente, como toda isometria $f: \mathbb{H}^{n} \rightarrow \mathbb{H}^{n}$ pode ser vista como uma transformação linear $A \in \mathrm{GL}(n+1, \mathbb{R})$, podemos estender $f$ como $\bar{f}: \mathbb{H}^{n} \cup \partial \mathbb{H}^{n} \rightarrow \mathbb{H}^{n} \cup \partial \mathbb{H}^{n}$ de modo a incluir o bordo, afinal $A$ leva retas do cone de luz em retas do cone de luz. Nesse sentido, $f$ pode assumir pontos fixos tanto em $\mathbb{H}^{n}$ quanto em $\partial \mathbb{H}^{n}$.

Teorema 6.4. Uma isometria $f$ de $\mathbb{H}^{n}$ é

- elíptica se $f$ possui algum ponto fixo em $\mathbb{H}^{n}$.

- parabólica se $f$ não possui ponto fixo e $\bar{f}$ possui exatamente um ponto fixo em $\partial \mathbb{H}^{n}$. 
- hiperbólica se $f$ não possui ponto fixo e $\bar{f}$ possui exatamente dois pontos fixos em $\partial \mathbb{H}^{n}$.

Exemplo 40. Relembre do exemplo anterior, onde estudamos o automorfismo linear em

$$
\operatorname{Pic}\left(\mathbb{P}_{P_{0} P_{1} P_{2}}^{2}\right)_{\mathbb{R}} \cong \mathbb{R}[\mathcal{L}] \oplus \mathbb{R}\left[\mathcal{E}_{0}\right] \oplus \mathbb{R}\left[\mathcal{E}_{1}\right] \oplus \mathbb{R}\left[\mathcal{E}_{2}\right]
$$

(induzido pelo automorfismo $\widetilde{\widetilde{\Phi}}$ definido no exemplo 38 ) dado pela matriz

$$
\left(\begin{array}{cccc}
2 & 1 & 1 & 1 \\
-1 & 0 & -1 & -1 \\
-1 & -1 & 0 & -1 \\
-1 & -1 & -1 & 0
\end{array}\right)
$$

Já sabemos que este automorfismo linear preserva a forma de assinatura $(1,3)$, o que também não é difícil de verificar algebricamente. Por definição, este automorfismo linear pertence a $O(1,3)$. Mais ainda, repare que o ponto $(1,0,0,0) \in \mathbb{H}^{3}$ é levado no ponto $(2,-1,-1,-1) \in \mathbb{H}^{3}$, portanto também pertence a $O^{+}(1,3)$, e induz uma isometria de $\mathbb{H}^{3}$. Mais especificamente, como o elemento $\frac{1}{\sqrt{6}}(-3,1,1,1) \in \mathbb{H}^{3}$ é um ponto fixo, trata-se de uma isometria elíptica. 


\section{7}

\section{A ação do Grupo de Cremona}

Até o momento, vimos que se $\varphi: \mathbb{P}^{2} \rightarrow \mathbb{P}^{2}$ é uma aplicação birracional conjugada a um automorfismo de $\mathbb{P}_{P_{0} P_{1} P_{2}}^{2}$, então $\varphi$ age em $\mathbb{H}^{3}$ como uma isometria. Porém, não é possível construir uma ação de todo o grupo $\operatorname{Cr}\left(\mathbb{P}^{2}\right)$ em $\mathbb{H}^{n}$. De fato, nem toda Transformação de Cremona é conjugada a um automorfismo de algum blowup $\mathbb{P}_{P_{1} \ldots P_{n}}^{2}$ (cf. (DILLER; FAVRE, 2001)).

Nosso objetivo agora será generalizar a ação de subgrupos de $\operatorname{Cr}\left(\mathbb{P}^{2}\right)$ em $\mathbb{H}^{n}$ descrita no Capítulo 6 de modo a obter uma ação de todo o grupo $\operatorname{Cr}\left(\mathbb{P}^{2}\right)$ em um certo espaço hiperbólico. Para isso, vamos construir o espaço de Picard-Manin de $\mathbb{P}^{2}$, que intuitivamente seria o espaço de Picard real se pudéssemos explodir todos os pontos de $\mathbb{P}^{2}$ e ainda os de seus blowups. $\mathrm{O}$ completamento do espaço de Picard-Manin ambienta um espaço hiperbólico de dimensão infinita $\mathbb{H}^{\infty}$ e com isso teremos uma ação de $\operatorname{Cr}\left(\mathbb{P}^{2}\right)$ por isometrias nesse $\mathbb{H}^{\infty}$. Referências para este Capítulo se encontram em (CANTAT; LAMY, 2013) e (LONJOU, 2016)

\section{1}

\section{O Espaço de Picard-Manin}

Definição 7.1. Um modelo de $\mathbb{P}^{2}$ é uma superfície projetiva não-singular $S$ com um morfismo birracional $\pi: S \rightarrow \mathbb{P}^{2}$. E se $\pi: S \rightarrow \mathbb{P}^{2}$ e $\pi^{\prime}: S^{\prime} \rightarrow \mathbb{P}^{2}$ são dois modelos de $\mathbb{P}^{2}$, dizemos que $S^{\prime}$ domina $S$ se a aplicação racional $\pi^{-1} \circ \pi^{\prime}: S^{\prime} \rightarrow S$ for um morfismo.

Note que quando $S^{\prime}$ domina $S$, o morfismo $\pi^{-1} \circ \pi^{\prime}: S^{\prime} \rightarrow S$ induz um pullback injetivo $\left(\pi^{-1} \circ \pi^{\prime}\right)^{*}: \operatorname{Pic}(S)_{\mathbb{R}} \hookrightarrow \operatorname{Pic}\left(S^{\prime}\right)_{\mathbb{R}}$, que preserva a forma de interseção. Dessa forma, podemos identificar $\operatorname{Pic}(S)_{\mathbb{R}}$ com sua imagem em $\operatorname{Pic}\left(S^{\prime}\right)_{\mathbb{R}}$.

Observe que dados $\pi_{1}: S_{1} \rightarrow \mathbb{P}^{2}$ e $\pi_{2}: S_{2} \rightarrow \mathbb{P}^{2}$ dois modelos de $\mathbb{P}^{2}$, pelo Teorema 4.3 existe uma superfície $S_{3}$ que domina $S_{1}$ e $S_{2}$. Sendo assim, podemos considerar as injetividades

$$
\operatorname{Pic}\left(S_{1}\right)_{\mathbb{R}} \hookrightarrow \operatorname{Pic}\left(S_{3}\right)_{\mathbb{R}} \text { e } \operatorname{Pic}\left(S_{2}\right)_{\mathbb{R}} \hookrightarrow \operatorname{Pic}\left(S_{3}\right)_{\mathbb{R}}
$$

dadas pelos pullbacks adequados. 


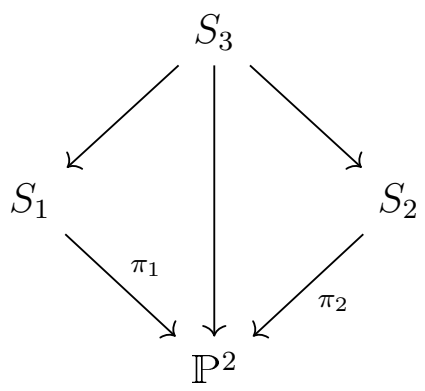

Se $D_{1} \in \operatorname{Div}\left(S_{1}\right)_{\mathbb{R}}$ e $D_{2} \in \operatorname{Div}\left(S_{2}\right)_{\mathbb{R}}$, dizemos que $\left[D_{1}\right] \in \operatorname{Pic}\left(S_{1}\right)_{\mathbb{R}}$ e $\left[D_{2}\right] \in \operatorname{Pic}\left(S_{2}\right)_{\mathbb{R}}$ são relacionados em $\bigsqcup_{S} \operatorname{Pic}(S)_{\mathbb{R}}\left(\operatorname{com} S\right.$ modelo de $\left.\mathbb{P}^{2}\right)$ quando são iguais em $\operatorname{Pic}\left(S_{3}\right)_{\mathbb{R}}$, isto é, quando

$$
\left(\pi_{1}^{-1} \circ \pi_{3}\right)^{*} D_{1} \sim\left(\pi_{2}^{-1} \circ \pi_{3}\right)^{*} D_{2}
$$

como divisores de $S_{3}$, e escrevemos $\left[D_{1}\right]_{S_{1}} \sim\left[D_{2}\right]_{S_{2}}$.

Definição 7.2. O Espaço de Picard Manin de $\mathbb{P}^{2}$ é o limite direto dos espaços $\operatorname{Pic}(S)_{\mathbb{R}}$, onde $S$ é modelo de $\mathbb{P}^{2}$, isto é,

$$
\mathcal{P} \mathcal{M}\left(\mathbb{P}^{2}\right)=\frac{\bigsqcup_{S} \operatorname{Pic}(S)_{\mathbb{R}}}{\sim}
$$

Dado $D \in \operatorname{Div}(S)_{\mathbb{R}}$ um divisor real de um modelo $S$ de $\mathbb{P}^{2}$, denotaremos a classe do elemento $[D] \in \operatorname{Pic}(S)_{\mathbb{R}}$ em $\mathcal{P} \mathcal{M}\left(\mathbb{P}^{2}\right)$ pela relação de equivalência descrita acima por sua letra minúscula $d$ em vez de $[[D]]$ para evitar notação carregada. Quando quisermos falar de um representante da classe $d$ em um modelo $S$ específico, usaremos a notação $[D]_{S}$ em vez de apenas $[D]$, para evitar ambiguidades.

Exemplo 41. Seja $\pi_{P}: S_{P} \rightarrow S$ o blowup de um modelo $S$ de $\mathbb{P}^{2}$ em $P \in S$, e divisor excepcional $E_{P}=\pi_{P}^{-1}(P)$. Seja $Q \in E_{P}$ e tome $\pi_{Q}: S_{P Q} \rightarrow S_{P}$ o blowup de $S_{P}$ em $Q$ e divisor excepcional $E_{Q}=\pi_{Q}^{-1}(Q)$. Podemos concluir que o pullback de $E_{P}$ por $\pi_{Q}$ é dado por $\mathcal{E}_{P}=E_{Q}+\widetilde{E_{P}}$. Em outras palavras, podemos dizer que em $\mathcal{P} \mathcal{M}\left(\mathbb{P}^{2}\right)$ temos o elemento $e_{P}$, dado pela classe de $\left[E_{P}\right]_{S_{P}} \sim\left[E_{Q}+\widetilde{E_{P}}\right]_{S_{P Q}}$

Observe que $\mathcal{P} \mathcal{M}\left(\mathbb{P}^{2}\right)$ contém $\operatorname{Pic}(S)_{\mathbb{R}}$ para qualquer modelo $S$ de $\mathbb{P}^{2}$. Isso permite que $\mathcal{P} \mathcal{M}\left(\mathbb{P}^{2}\right)$ herde uma forma de interseção, definida assim:

Sejam $d$ e $d^{\prime}$ em $\mathcal{P} \mathcal{M}\left(\mathbb{P}^{2}\right)$, então extiste um modelo $S_{1}$ de $\mathbb{P}^{2}$ onde podemos encontrar $[D]_{S_{1}},\left[D^{\prime}\right]_{S_{1}} \in \operatorname{Pic}\left(S_{1}\right)$ que representem essas classes. Então definimos $d \cdot d^{\prime}=[D]_{S_{1}} \cdot\left[D^{\prime}\right]_{S_{1}}$, onde o segunto produto é referente à forma de interseção em $S_{1}$.

Note que essa definição não depende da escolha do modelo, pois se outro modelo $S_{2}$ contém seus prórios $[D]_{S_{2}},\left[D^{\prime}\right]_{S_{2}} \in \operatorname{Pic}\left(S_{2}\right)$, então existe 
uma superfície $S_{3}$ que domina $S_{1}$ e $S_{2}$ contendo seus próprios representantes $[D]_{S_{3}},\left[D^{\prime}\right]_{S_{3}} \in \operatorname{Pic}\left(S_{3}\right)$. Como o pullback preserva forma de interseção, temos que $[D]_{S_{1}} \cdot\left[D^{\prime}\right]_{S_{1}}=[D]_{S_{3}} \cdot\left[D^{\prime}\right]_{S_{3}}=[D]_{S_{2}} \cdot\left[D^{\prime}\right]_{S_{2}}$.

Vale relembrar que para qualquer superfície lisa $S$ e $P \in S$ um ponto, o grupo de Picard do blowup satisfaz $\operatorname{Pic}\left(S_{P}\right) \cong \operatorname{Pic}(S) \oplus \mathbb{Z}[\mathcal{E}]$, onde $\mathcal{E}$ é o divisor excepcional do blowup. A inclusão $\operatorname{Pic}(S) \subset \operatorname{Pic}\left(S_{P}\right)$ é vista como descrito acima, usando o pullback.

De forma análoga, temos que $\operatorname{Pic}\left(S_{P}\right)_{\mathbb{R}} \cong \operatorname{Pic}(S)_{\mathbb{R}} \oplus \mathbb{R}[\mathcal{E}]$, e como espaço vetorial, podemos escolher uma base de $\operatorname{Pic}(S)_{\mathbb{R}}$, tomar seu pullback e acrescentar $[\mathcal{E}]$ para obter uma base para $\operatorname{Pic}\left(S_{P}\right)_{\mathbb{R}}$.

Como sabemos pelo Teorema 4.1 que todo modelo de $\mathbb{P}^{2}$ é uma composição de finitos blowups, por indução cada $\operatorname{Pic}(S)_{\mathbb{R}}$ tem como base o pullback de uma reta em $\mathbb{P}^{2}$ e também o pullback de cada divisor excepcional de cada blowup realizado. Ou seja, $\operatorname{Pic}\left(\mathbb{P}_{P_{1} \ldots P_{n}}^{2}\right)_{\mathbb{R}}=\left\langle[\mathcal{L}],\left[\mathcal{E}_{1}\right], \ldots,\left[\mathcal{E}_{n}\right]\right\rangle$.

Já para o espaço vetorial $\mathcal{P} \mathcal{M}\left(\mathbb{P}^{2}\right)$, teremos uma base infinita, e precisamos incluir todos os divisores excepcionais tomando o cuidado de não repetir elementos que sejam relacionados. Para construir uma base de forma precisa, considere a seguinte definição.

Definição 7.3. O espaço bolha de $\mathbb{P}^{2}$ é a união dos modelos de $\mathbb{P}^{2}$ com a seguinte relação de equivalência: dados $P_{1} \in S_{1}$ e $P_{2} \in S_{2}$, teremos $P_{1} \sim P_{2}$ se $\pi_{2}^{-1} \circ \pi_{1}: S_{1} \rightarrow S_{2}$ é um isomorfismo de uma vizinhança de $P_{1}$ em uma vizinhança de $P_{2}$ tal que $P_{1} \mapsto P_{2}$. Em outros termos,

$$
\mathcal{B}\left(\mathbb{P}^{2}\right)=\frac{\bigsqcup S \text { modelo de } \mathbb{P}^{2}}{\sim} .
$$

Informalmente, dizemos que $\mathcal{B}\left(\mathbb{P}^{2}\right)$ é o espaço dos pontos de $\mathbb{P}^{2}$ e dos pontos infinitamente próximos a ele, que são os pontos em divisores excepcionais de explosões de $\mathbb{P}^{2}$.

Com essa nova definição, podemos construir uma base para $\mathcal{P} \mathcal{M}\left(\mathbb{P}^{2}\right)$ dada por $\{l\} \cup\left\{e_{P} \mid P \in \mathcal{B}\left(\mathbb{P}^{2}\right)\right\}$. Como consequência, os elementos de $\mathcal{P} \mathcal{M}\left(\mathbb{P}^{2}\right)$ podem ser sempre escritos como combinação linear finita dos elementos da base.

É fácil ver que tal base é ortogonal com respeito à forma de interseção. Assim, sejam

$$
d=x_{0} l+\sum_{P \in \mathcal{B}\left(\mathbb{P}^{2}\right)} x_{P} e_{P}, d^{\prime}=y_{0} l+\sum_{P \in \mathcal{B}\left(\mathbb{P}^{2}\right)} y_{P}^{\prime} e_{P}
$$

elementos de $\mathcal{P} \mathcal{M}\left(\mathbb{P}^{2}\right)$, então 


$$
d \cdot d^{\prime}=x_{0} y_{0}-\sum_{P \in \mathcal{B}\left(\mathbb{P}^{2}\right)} x_{P} y_{P}
$$

Tal forma bilinear pode remeter ao Capítulo anterior, porém o espaço $\mathcal{P} \mathcal{M}\left(\mathbb{P}^{2}\right)$ falha em ser completo. Seu completamento é conhecido como o espaço de Picard-Manin completo de $\mathbb{P}^{2}$.

Definição 7.4. O espaço de Picard-Manin completo de $\mathbb{P}^{2}$ é definido como

$$
\overline{\mathcal{P} \mathcal{M}}\left(\mathbb{P}^{2}\right)=\left\{\lambda_{0} l+\sum_{P \in \mathcal{B}\left(\mathbb{P}^{2}\right)} \lambda_{P} e_{p} \mid \lambda_{0}, \lambda_{P} \in \mathbb{R} \forall P e \sum_{P \in \mathcal{B}\left(\mathbb{P}^{2}\right)} \lambda_{P}^{2}<\infty\right\} .
$$

Agora que temos um espaço completo, juntamente com a forma bilinear dada por (7.1), podemos relacionar com um espaço hiperbólico de dimensão infinita.

\section{2}

\section{O modelo do hiperbolóide em dimensão infinita}

Suponha que temos um conjunto infinito $I$ e considere o espaço vetorial

$$
V=\left\{\left(v_{i}\right)_{i \in I \sqcup\{0\}} \mid \sum_{i \in I} v_{i}^{2}<\infty\right\} .
$$

Para que a soma dos quadrados das coordenadas seja finito, está implícito que deve existir uma quantidade enumerável de coordenadas não nulas.

A forma bilinear simétrica de assinatura $(1, \infty)$

$$
\left(u_{i}\right)_{i \in I \sqcup\{0\}} \cdot\left(v_{i}\right)_{i \in I \sqcup\{0\}}=u_{0} v_{0}-\sum_{i \in I} u_{i} v_{i}
$$

está bem definida graças à desigualdade de Cauchy-Schwarz e podemos então definir o análogo da folha de hiperbolóide visto no Capítulo 6 como

$$
\mathbb{H}^{\infty}=\left\{v=\left(v_{i}\right)_{i \in I \sqcup\{0\}} \in V \mid v \cdot v=1 \text { e } v_{0}>0\right\} .
$$

O espaço $\mathbb{H}^{\infty}$ munido da distância $\mathrm{d}(u, v)=\cosh ^{-1}(u \cdot v)$ é um espaço métrico completo de dimensão infinita. Além disso, se a interseção de $\mathbb{H}^{\infty}$ com um subespaço vetorial $W \subset V$ de dimensão $n+1$ é não vazia, então $\mathbb{H}^{\infty} \cap W \cong \mathbb{H}^{n}$ é uma cópia do espaço hiperbólico de dimensão $n$ estudado no Capítulo anterior.

Em particular, dados dois pontos quaisquer $u, v \in \mathbb{H}^{\infty}$, existe uma única geodésica contendo ambos, dada por $\mathbb{H}^{\infty} \cap \operatorname{Span}(u, v)$. Se $u, v, w \in \mathbb{H}^{\infty}$, o triângulo geodésico que os contém como vértices está contido em

$$
\mathbb{H}^{\infty} \cap \operatorname{Span}(u, v, w),
$$


que é por sua vez isomorfo a $\mathbb{H}^{2}$. Por isso, o espaço $\mathbb{H}^{\infty}$ é também CAT(1) e $\delta$-hiperbólico para o mesmo $\delta$. Lembramos que as definições de CAT(1) e $\delta$-hiperbolicidade não são relevantes no nosso estudo, mas podem ser encontradas em (PAUPERT, 2016). Em breve, vamos precisar que o nosso espaço $\overline{\mathcal{P} \mathcal{M}}\left(\mathbb{P}^{2}\right)$ tenha essas duas propriedades para satisfazermos as hipóteses do Teorema 8.2.

Todas as Definições, Proposições e Teoremas da Seção 6.3 (referente a isometrias de $\mathbb{H}^{n}$ ) se mantêm para isometrias de $\mathbb{H}^{\infty}$, a menos da troca de $n$ por $\infty$. Vamos acrescentar também um pouco de teoria a respeito de isometrias hiperbólicas, que nos será útil no Capítulo 8.

Definição 7.5. Seja $\mathbb{H}^{\infty}$ um espaço hiperbólico de dimensão infinita e $h \epsilon$ $\operatorname{Isom}\left(\mathbb{H}^{\infty}\right)$ uma isometria hiperbólica. O eixo de $h$ é definido por

$$
\operatorname{Eixo}(h)=\left\{x \in \mathbb{H}^{\infty} \mid d(x, h(x))=L(h)\right\}
$$

Relembre que $L(h)$ é o ínfimo das distâncias entre $x$ e $h(x)$, ou seja, $\operatorname{Eixo}(h)$ é o conjunto dos pontos de $\mathbb{H}^{\infty}$ que realizam esse ínfimo. O fato de $h$ ser hiperbólica implica que $\operatorname{Eixo}(h) \neq \varnothing$ e que $L(h)>0$.

Na verdade, o eixo de uma isometria hiperbólica $h$ consiste de uma única geodésica, onde a restrição de $h$ se comporta como uma translação de comprimento $L(h)$. Mais precisamente, se $\gamma: \mathbb{R} \rightarrow \operatorname{Eixo}(h)$ é uma parametrização isométrica dessa geodésica, então temos que $h(x)=\gamma\left(\gamma^{-1}(x)+L(h)\right)$ para todo $x \in \operatorname{Eixo}(h)$ ou $h(x)=\gamma\left(\gamma^{-1}(x)-L(h)\right)$ para todo $x \in \operatorname{Eixo}(h)$.

Supondo que escolhemos $\gamma$ de modo que $h(x)=\gamma\left(\gamma^{-1}(x)+L(h)\right)$ para todo $x \in \operatorname{Eixo}(h)$, podemos definir $t^{-}=\gamma(-\infty) \in \partial \mathbb{H}^{\infty}$ e $t^{+}=\gamma(+\infty) \in \partial \mathbb{H}^{\infty}$, ditos os terminais de $\operatorname{Eixo}(h)$, repulsivo e atrativo respectivamente. Temos também uma ordem natural dos pontos em $\operatorname{Eixo}(h)$ : dizemos que $x<y$ quando $\gamma^{-1}(x)<\gamma^{-1}(y)$. Também vale que para qualquer ponto $x \in \mathbb{H}^{\infty}$, as sequências $h^{-n}(x)$ e $h^{n}(x)$ convergem respectivamente para $t^{-}$e $t^{+}$.

Denotaremos por $\mathbb{H}^{\infty}\left(\mathbb{P}^{2}\right)$ a folha do hiperbolóide como acima quando $V=\overline{\mathcal{P} M}\left(\mathbb{P}^{2}\right)$ e $I=\mathcal{B}\left(\mathbb{P}^{2}\right)$.

\section{3}

\section{A ação}

Nosso objetivo agora será construir a ação de $\operatorname{Cr}\left(\mathbb{P}^{2}\right)$ em $\mathbb{H}^{\infty}\left(\mathbb{P}^{2}\right)$.

Seja $\varphi:\left(\mathbb{P}^{2}\right)_{\alpha} \rightarrow\left(\mathbb{P}^{2}\right)_{\omega}$ uma Transformação de Cremona (os índices $\alpha \mathrm{e}$ $\omega$ estão apenas diferenciando a origem e o destino da aplicação racional, para evitar confusão) e $d \in \mathcal{P} \mathcal{M}\left(\mathbb{P}^{2}\right)$. Tome $\pi: S \rightarrow\left(\mathbb{P}^{2}\right)_{\omega}$ um modelo de $\left(\mathbb{P}^{2}\right)_{\omega}$ tal que $S$ possua como $\mathbb{R}$-divisor um representante $[D]_{S}$ de $d$. 
Agora considere a aplicação birracional dada por $\pi^{-1} \circ \varphi:\left(\mathbb{P}^{2}\right)_{\alpha} \rightarrow S$ e nela vamos aplicar o Teorema 4.3. Então sejam $\widetilde{\varphi}: S^{\prime} \rightarrow S$ e $\tau: S^{\prime} \rightarrow\left(\mathbb{P}^{2}\right)_{\alpha}$ composições de blowups tais que $\pi \circ \widetilde{\varphi}=\varphi \circ \tau$.

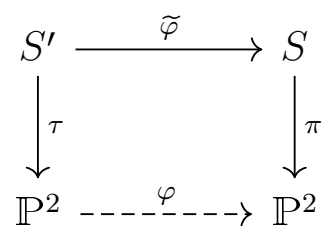

Considere

$$
\widetilde{\varphi}^{*}: \operatorname{Pic}(S)_{\mathbb{R}} \hookrightarrow \operatorname{Pic}\left(S^{\prime}\right)_{\mathbb{R}}
$$

e $[D]_{S} \mapsto\left[D^{\prime}\right]_{S^{\prime}}$. Definimos $\varphi^{*}: \mathcal{P} \mathcal{M}\left(\mathbb{P}^{2}\right) \rightarrow \mathcal{P} \mathcal{M}\left(\mathbb{P}^{2}\right)$ dada por

$$
\varphi^{*} d=\left[\left[D^{\prime}\right]_{S^{\prime}}\right] \in \mathcal{P} \mathcal{M}\left(\mathbb{P}^{2}\right)
$$

A aplicação $\varphi \mapsto \varphi^{*}$ é, em algum sentido, uma generalização do pullback para Transformações de Cremona, e também é contravariante. Perceba que a escolha das superfícies $S$ e $S^{\prime}$ acima não são relevantes.

Exemplo 42. Vamos estudar a imagem de $l \in \mathcal{P} \mathcal{M}\left(\mathbb{P}^{2}\right)$ por $\varphi^{*}$, onde $\varphi:\left(\mathbb{P}^{2}\right)_{\alpha} \rightarrow$ $\left(\mathbb{P}^{2}\right)_{\omega}$ é uma Transformação de Cremona qualquer de grau $N$.

Para obter um representante de $l$ basta tomar $S=\left(\mathbb{P}^{2}\right)_{\omega}$ e uma reta qualquer $L \subset\left(\mathbb{P}^{2}\right)_{\omega}$. A resolução de $\varphi$ é dada pelas composições de morfismos $\tau: S^{\prime} \rightarrow\left(\mathbb{P}^{2}\right)_{\alpha}$ e $\widetilde{\varphi}: S^{\prime} \rightarrow\left(\mathbb{P}^{2}\right)_{\omega}$. Queremos saber como é o elemento $\widetilde{\varphi}^{*}[L]_{\mathbb{P}^{2}} \in \operatorname{Pic}\left(S^{\prime}\right)$ em termos da base $\operatorname{de} \operatorname{Pic}\left(S^{\prime}\right)$ dada por $\tau$.

Supondo que $L$ não contenha nenhum ponto de indeterminação de $\varphi$ ou $\varphi^{-1}$, então $\varphi^{-1}(L)$ é uma curva $C$ de grau $N$ em $\left(\mathbb{P}^{2}\right)_{\alpha}$ contendo todos os pontos $P_{1}, \ldots, P_{r}$ de indeterminação de $\varphi$, com multiplicidade $m_{i} \geq 1, i=1, \ldots, r$.

Além disso, como a transformada estrita $\widetilde{L}$ de $L$ por $\widetilde{\varphi}$ é isomorfa a $L$, que é irredutível, teremos $\widetilde{L}=\widetilde{C}$, onde $\widetilde{C}$ é a transformada estrita de $C$ por $\tau$. Em outros termos,

$$
\widetilde{\varphi}^{\star} L=\widetilde{L}=\widetilde{C} \sim N \mathcal{L}-\sum_{i=1}^{r} m_{i} \mathcal{E}_{i}
$$

e então,

$$
\varphi^{*} l=N l-\sum_{i=1}^{r} m_{i} e_{i} \in \mathcal{P} \mathcal{M}\left(\mathbb{P}^{2}\right)
$$

A aplicação $\varphi \mapsto \varphi^{*}$ não é exatamente a ação que vamos utilizar, simplesmente porque preferiremos uma aplicação que seja covariante. A transformação linear $\varphi^{*}$ admite uma inversa linear construída de forma parecida a seguir, e que é covariante.

Seja $d \in \mathcal{P} \mathcal{M}\left(\mathbb{P}^{2}\right)$ e $\pi: S \rightarrow\left(\mathbb{P}^{2}\right)_{\alpha}$ um modelo de $\left(\mathbb{P}^{2}\right)_{\alpha}$ tal que $S$ possua como $\mathbb{R}$-divisor um representante $[D]_{S}$ de $d$. A aplicação birracional 


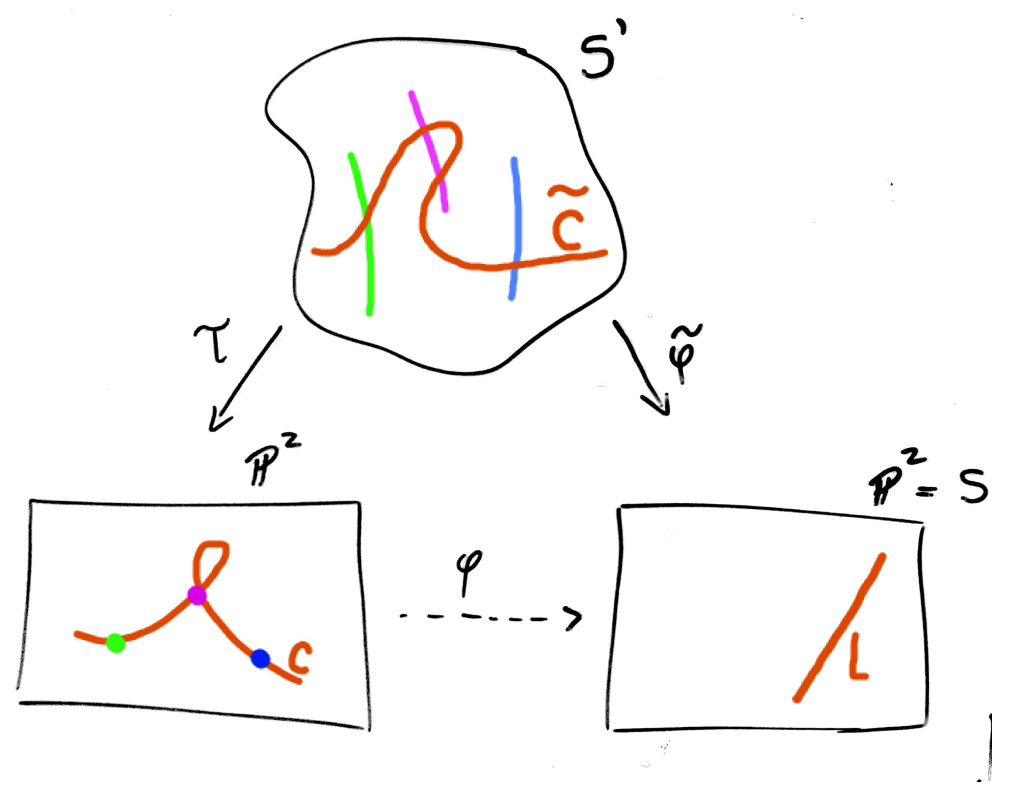

$\varphi \circ \pi: S \rightarrow\left(\mathbb{P}^{2}\right)_{\omega}$ se aplica ao Teorema 4.3, então sejam $\widetilde{\varphi^{-1}}: S^{\prime} \rightarrow S$ e $\tau: S^{\prime} \rightarrow\left(\mathbb{P}^{2}\right)_{\omega}$ morfismos birracionais tais que $\pi \circ \widetilde{\varphi^{-1}}=\varphi^{-1} \circ \tau$.

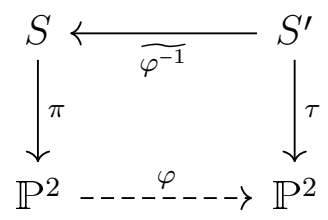

Considere

$$
{\widetilde{\varphi^{-1}}}^{*}: \operatorname{Pic}(S)_{\mathbb{R}} \hookrightarrow \operatorname{Pic}\left(S^{\prime}\right)_{\mathbb{R}}
$$

e $[D]_{S} \mapsto\left[D^{\prime}\right]_{S^{\prime}}$. Definimos $\varphi_{*}: \mathcal{P} \mathcal{M}\left(\mathbb{P}^{2}\right) \rightarrow \mathcal{P} \mathcal{M}\left(\mathbb{P}^{2}\right)$ dada por

$$
\varphi_{\star} d=\left[\left[D^{\prime}\right]_{S^{\prime}}\right] \in \mathcal{P} \mathcal{M}\left(\mathbb{P}^{2}\right) .
$$

Ambas $\varphi^{*}$ e $\varphi_{*}$ admitem uma extensão linear $\overline{\mathcal{P} \mathcal{M}}\left(\mathbb{P}^{2}\right) \rightarrow \overline{\mathcal{P M}}\left(\mathbb{P}^{2}\right)$, sendo portanto automorfismos lineares de $\overline{\mathcal{P M}}\left(\mathbb{P}^{2}\right)$ que preservam a forma de interseção. Além disso, pelo exemplo anterior podemos verificar que $\varphi^{*}$ (e consequentemente $\left.\varphi_{*}\right)$ preservam a folha de hiperbolóide $\mathbb{H}^{\infty}\left(\mathbb{P}^{2}\right)$, já que

$$
\varphi^{*} l=N[\mathcal{L}]-\sum_{i=1}^{r} m_{i}\left[\mathcal{E}_{i}\right]
$$

$\operatorname{com} N=\operatorname{deg}(\varphi)$ inteiro positivo.

Isso significa que as restrições de $\varphi^{*}$ e $\varphi_{*}$ à folha de hiperbolóide $\mathbb{H}^{\infty}\left(\mathbb{P}^{2}\right)$ são isometrias de $\mathbb{H}^{\infty}\left(\mathbb{P}^{2}\right)$. A tão mencionada ação de $\operatorname{Cr}\left(\mathbb{P}^{2}\right)$ em $\mathbb{H}^{\infty}\left(\mathbb{P}^{2}\right)$ é dada por $\varphi \mapsto \varphi_{*}$. Mais precisamente, temos um homomorfismo de grupos $\operatorname{Cr}\left(\mathbb{P}^{2}\right) \rightarrow \operatorname{Isom}\left(\mathbb{H}^{\infty}\left(\mathbb{P}^{2}\right)\right)$.

Repare que se $\varphi$ é um isomorfismo de um aberto contendo o ponto $P \in \mathbb{P}^{2}$, 
então $\varphi_{\star} e_{P}=e_{\varphi(P)}$. Isso implica que a ação é fiel, isto é, que o homomorfismo acima é injetivo. 


\section{8}

\section{Não simplicidade do Grupo de Cremona}

Em (CANTAT; LAMY, 2013), os autores provam que o Grupo de Cremona $\operatorname{Cr}\left(\mathbb{P}_{k}^{2}\right)$ não é simples quando $k$ for um corpo algebricamente fechado. A demonstração envolve a ação de grupo $\operatorname{Cr}\left(\mathbb{P}_{k}^{2}\right) \rightarrow \operatorname{Isom}\left(\mathbb{H}^{\infty}\left(\mathbb{P}_{k}^{2}\right)\right)$ que construímos no Capítulo anterior e alguns resultados de Teoria Geométrica dos Grupos, provadas por eles mesmos no próprio artigo.

Em (LONJOU, 2016), a autora generaliza o resultado para qualquer corpo $k$. A demonstração é baseada na ação de grupo $\operatorname{Cr}\left(\mathbb{P}_{k}^{2}\right) \rightarrow$ $\operatorname{Isom}\left(\mathbb{H}^{\infty}\left(\mathbb{P}_{\bar{k}}^{2}\right)\right)$, porém é bem mais curta e simples que a demonstração de Cantat e Lamy. A razão é provavelmente o avanço na área de Teoria Geométrica dos Grupos no intervalo entre os dois artigos. De fato, Lonjou usa um resultado recente na época: o Teorema 8.2, enunciado mais à frente.

Neste Capítulo, veremos um resumo de como Lonjou provou a não simplicidade do Grupo de Cremona em (LONJOU, 2016), com o objetivo de exemplificar o poder da ação que construímos.

Vamos retomar a notação $\mathbb{P}_{k}^{2}$ para lembrarmos do corpo de base do plano projetivo em questão. Como o resultado vale para qualquer corpo $k$, vamos abandonar a restrição de ser algebricamente fechado, e eventualmente usaremos seu fecho algébrico denotado por $\bar{k}$.

Note que as definições de morfismo e aplicação racional e birracional ainda fazem sentido mesmo se o corpo $k$ não for algebricamente fechado (definições 3.1, 3.4 e 3.6). Desta forma podemos tratar do grupo $\operatorname{Cr}\left(\mathbb{P}_{k}^{2}\right)$ das Transformações de Cremona de $\mathbb{P}_{k}^{2}$. Mas é fácil observar que $\operatorname{Cr}\left(\mathbb{P}_{k}^{2}\right) \subset \operatorname{Cr}\left(\mathbb{P}_{k}^{2}\right)$, por isso temos $\operatorname{Cr}\left(\mathbb{P}_{k}^{2}\right)$ também agindo no espaço $\delta$-hiperbólico $\mathbb{H}^{\infty}\left(\mathbb{P}_{\bar{k}}^{2}\right)$. Nosso objetivo é provar o seguinte Teorema:

Teorema 8.1. Seja $k$ um corpo qualquer. O Grupo de Cremona $\operatorname{Cr}\left(\mathbb{P}_{k}^{2}\right)$ não é simples.

Considere o seguinte Teorema, de Teoria Geométrica dos Grupos:

Teorema 8.2 ((DAHMANI; GUIRARDEL; OSIN, 2017)). Sejam G um grupo que age por isometrias em um espaço $\delta$-hiperbólico. Seja $h$ um elemento hiperbólico de $G$ que satisfaz a propriedade WPD. Então para n suficientemente grande, o subgrupo normal $\left\langle\left\langle h^{n}\right\rangle\right\rangle$ de $G$ é próprio.

Estamos interessados no caso $G=\operatorname{Cr}\left(\mathbb{P}_{k}^{2}\right)$. Pelo Teorema 8.2 acima, basta encontrarmos algum elemento hiperbólico em $\operatorname{Cr}\left(\mathbb{P}_{k}^{2}\right)$ que satisfaz a propriedade WPD (proposta por Bestvina e Fujiwara em (BESTVINA; FUJIWARA, 
2002)), definida na próxima Seção. Com o Teorema 8.3 a seguir, Lonjou conclui a demonstração do Teorema 8.1.

Teorema 8.3 ((LONJOU, 2016)). Seja $n \geq 2$ tal que char $(k) \nmid n$. Considerando a ação de $\operatorname{Cr}\left(\mathbb{P}_{k}^{2}\right)$ por isometrias em $\mathbb{H}^{\infty}\left(\mathbb{P}_{k}^{2}\right)$, o elemento $h_{n} \in \operatorname{Cr}\left(\mathbb{P}_{k}^{2}\right)$ dado por $(x: y: z) \mapsto\left(y z^{n-1}: y^{n}-x z^{n-1}: z^{n}\right)$ é hiperbólico e satisfaz a propriedade $W P D$.

\section{1}

\section{A propriedade WPD}

Definição 8.1. Dado um grupo $G$ que age por isometrias em um espaço métrico $(X, d)$ e $A \subset X$ um subconjunto qualquer, definimos o estabilizador de $A$ por $G$ a menos de $\varepsilon$ por

$$
\operatorname{Stab}_{\varepsilon} A=\{g \in G \mid d(a, g \cdot a) \leq \varepsilon \forall a \in A\} .
$$

Definição 8.2. Dado um grupo $G$ que age por isometrias em um espaço métrico $(X, d)$, dizemos que $g \in G$ satisfaz a propriedade WPD (weakly properly discontinuous) se existe $y \in X$ tal que para todo $\varepsilon>0$ existe um número $N \in \mathbb{N}$ tal que $\operatorname{Stab}_{\varepsilon}\left\{y, g^{N} \cdot y\right\}$ é finito.

Proposição 8.1. Dado um grupo $G$ que age por isometrias em um espaço métrico $(X, d)$ e $g \in G$, as seguintes propriedades são equivalentes:

- Existe $y \in X$ tal que para todo $\varepsilon>0$ existe um número $N \in \mathbb{N}$ tal que $\operatorname{Stab}_{\varepsilon}\left\{y, g^{N} \cdot y\right\}$ é finito.

- Para todo $x \in X$ e para todo $\varepsilon>0$ existe um número $N \in \mathbb{N}$ tal que $\operatorname{Stab}_{\varepsilon}\left\{x, g^{N} \cdot x\right\}$ é finito.

Prova. Seja $y$ como no primeiro item, e $x \in X, \varepsilon>0$ quaisquer. Sejam $\varepsilon^{\prime}=2 \mathrm{~d}(x, y)+\varepsilon$ e $N$ tal que $\operatorname{Stab}_{\varepsilon^{\prime}}\left\{y, g^{N} \cdot y\right\}$ é finito. Por desigualdade triangular,

$$
\operatorname{Stab}_{\varepsilon}\left\{x, g^{N} \cdot x\right\} \subset \operatorname{Stab}_{\varepsilon^{\prime}}\left\{y, g^{N} \cdot y\right\}
$$

Lonjou conseguiu enfraquecer a condição de ser WPD com a Proposição 8.2 enunciada a seguir, com o objetivo de mostrar que o elemento $h_{n}$ proposto por ela satisfazia essa propriedade. Mas antes, relembre da Definição 7.5:

Seja $\mathbb{H}^{\infty}$ um espaço hiperbólico de dimensão infinita e $h \in \operatorname{Isom}\left(\mathbb{H}^{\infty}\right)$ uma isometria hiperbólica. O eixo de $h$ é definido por

$$
\operatorname{Eixo}(h)=\left\{x \in \mathbb{H}^{\infty} \mid \mathrm{d}(x, h(x))=L(h)\right\} .
$$


Proposição 8.2. Dado um grupo $G$ que age por isometrias em um espaço hiperbólico $\mathbb{H}^{\infty}$ e $h \in G$ um elemento hiperbólico, as seguintes propriedades são equivalentes:

- Existe $y \in \operatorname{Eixo}(h)$ tal que para todo $\varepsilon>0$ existe um número $N \in \mathbb{N}$ tal que $\operatorname{Stab}_{\varepsilon}\left\{y, h^{N}(y)\right\}$ é finito.

- Existem $z \in \operatorname{Eixo}(h), \eta>0$ e $n, k \in \mathbb{N}$ tais que $\operatorname{Stab}_{\eta}\left\{h^{-k}(z), h^{n}(z)\right\}$ é finito.

A demonstração desta Proposição se encontra em (LONJOU, 2016) e usa apenas argumentos elementares de Geometria Hiperbólica. Com isso, só precisamos verificar que $h_{n}$ é um elemento hiperbólico que satisfaz o segundo item.

\section{2}

\section{Os elementos $h_{n}$}

Relembre que estamos interessados em entender a Transformação de Cremona $h_{n} \in \operatorname{Cr}\left(\mathbb{P}_{k}^{2}\right)$ dada por

$$
(x: y: z) \mapsto\left(y z^{n-1}: y^{n}-x z^{n-1}: z^{n}\right) .
$$

Sejam $a: \mathbb{P}_{\bar{k}}^{2} \rightarrow \mathbb{P}_{\bar{k}}^{2}$ o automorfismo tal que $(x: y: z) \mapsto(y: x: z)$ e $j_{n}: \mathbb{P}_{\bar{k}}^{2} \rightarrow$ $\mathbb{P}_{\bar{k}}^{2}$ a Transformação de Cremona tal que $(x: y: z) \mapsto\left(y^{n}-x z^{n-1}: y z^{n-1}: z^{n}\right)$. Então temos que $h_{n}=a \circ j_{n}$ e $h_{n}^{-1}=a^{-1} \circ h_{n} \circ a$.

A transformação $j_{n}$ é dita de de Jonquière, um tipo de Transformação de Cremona que preserva algum feixe de retas contendo um ponto fixado. Note que $j_{n}$ tem exatamente um ponto de indeterminação, assim como $h_{n}$, a saber $P_{0}=(1: 0: 0)$.

É sabido ((KOLLáR; SMITH; CORTI, 2004) definição 2.28, por exemplo) que para resolver essa indeterminação será preciso tomar o blowup de $\mathbb{P}_{k}^{2} \mathrm{em}$ $P_{0}$, em seguida tomar o blowup dessa superfície em um ponto $P_{1} \in E_{0}$ e assim por diante até o ponto $P_{2 n-2} \in E_{2 n-3}$. Cada ponto $P_{k}$ é tomado no divisor excepcional imediatamente anterior e dizemos que cada $P_{l}$ é infinitamente próximo a $P_{l-1}$. Exceto pelos pontos $P_{0}$ e $P_{1}$, todos os outros dependem de $n$. (Aqui a notação difere dos Capítulos anteriores, onde $P_{0}, P_{1}, P_{2}$ representavam os pontos coordenados de $\mathbb{P}_{k}^{2}$.)

Como a é um automorfismo de $\mathbb{P}_{k}^{2}$, para resolver as indeterminações de $h_{n}$, precisamos tomar blowups nos mesmos pontos $P_{l}$. Já para resolver as indeterminações de $h_{n}^{-1}$, tomamos os blowups nos pontos $Q_{l}$ simétricos aos pontos $P_{l}$. 
Para determinar que $h_{n *}$ é uma isometria hiperbólica, vamos usar o seguinte fato encontrado em (CANTAT; LAMY, 2013) Teorema 4.4, Observação 4.5: dada uma Transformação de Cremona $\varphi$, então $L\left(\varphi_{*}\right)=\log (\lambda(\varphi))$, onde

$$
\lambda(\varphi)=\limsup _{n \rightarrow \infty}\left[\operatorname{deg}\left(\varphi^{n}\right)\right]^{1 / n}
$$

é um invariante por conjugação conhecido como grau dinâmico de $\varphi$. Podemos concluir que $\varphi_{*}$ é uma isometria hiperbólica se e só se $\lambda(\varphi)>1$.

Note que não vale em geral que

$$
\operatorname{deg}\left(\varphi^{i}\right)=\operatorname{deg}(\varphi)^{i}
$$

Por exemplo, a Transformação Quadrática Padrão é tal que $\operatorname{deg}(\Phi)=2 \mathrm{e}$ $\operatorname{deg}\left(\Phi^{2}\right)=1$.

O que ocorre é que a fórmula $(x: y: z) \mapsto\left(x^{2} y z: x y^{2} z: x y z^{2}\right)$ da composição $\Phi^{2}$ tem seus três polinômios homogêneos divisíveis por $x y z$, o que reduz o grau de $\Phi^{2}$ em 3. Geometricamente, isso significa a inclusão $\Phi(\mathcal{Z}(x y z)) \subset$ $\operatorname{Ind}(\Phi)$, onde $\operatorname{Ind}(\Phi)=\{(1: 0:),(0: 1: 0),(0: 0: 1)\}$ é o conjunto de pontos de indeterminação de $\Phi$.

Ou seja, para que a equação 8.1 não ocorra, é necessário que exista alguma curva $C \subset \mathbb{P}_{k}^{2}$ tal que $\varphi^{i-1}(C) \subset \operatorname{Ind}(\varphi)$. Isso implica que no caso de $\varphi=h_{n}$ de fato temos 8.1. Isso porque seu único ponto de indeterminação $P_{0}=(1: 0: 0)$ não pertence à imagem de $h_{n}$, tornando impossível a condição geométrica exigida.

Com isso, teremos $\lambda\left(h_{n}\right)=n>1$, e concluímos que $h_{n *}$ é uma isometria hiperbólica. Além disso, Lonjou calculou os pontos $t^{-}$e $t^{+}$tomando os limites $h_{n_{*}}^{-m} \cdot l$ e $h_{n_{*}}^{m} \cdot l$ quando $m \rightarrow \infty$, onde $l \in \mathbb{H}^{\infty}\left(\mathbb{P}_{k}^{2}\right) \subset \overline{\mathcal{P} \mathcal{M}}\left(\mathbb{P}_{k}^{2}\right)$ é a classe de $[L] \in \operatorname{Pic}\left(\mathbb{P}_{k}^{2}\right)$ em $\overline{\mathcal{P} \mathcal{M}}\left(\mathbb{P}_{k}^{2}\right)$.

Proposição 8.3. Seja $w_{n}$ a projeção de l em Eixo $\left(h_{n *}\right)$. Existe $\varepsilon_{n}$ tal que todo elemento de $\operatorname{Stab}_{\varepsilon_{n}}\left\{w_{n}\right\} \subset \operatorname{Cr}\left(\mathbb{P}_{\bar{k}}^{2}\right)$ é um automorfismo de $\mathbb{P}_{\bar{k}}^{2}$. 


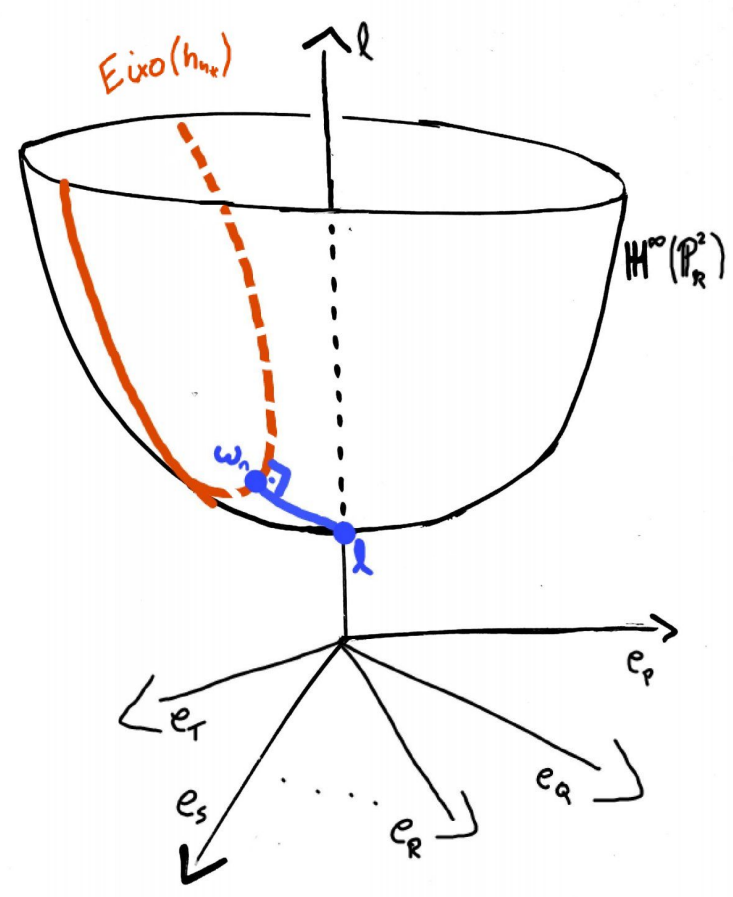

A demonstração da Proposição 8.3 em (LONJOU, 2016) também apenas usa Geometria Hiperbólica elementar. Antes de prosseguir, uma última Proposição vai ser útil na demonstração do Teorema 8.3.

Proposição 8.4. Seja $G$ um grupo agindo por isometrias em um espaço hiperbólico $\mathbb{H}^{\infty}$. Considere quatro pontos $x<y<y^{\prime}<x^{\prime}$ em uma mesma geodésica. Então para todo $\varepsilon>0$

$$
\operatorname{Stab}_{\varepsilon}\left\{x, x^{\prime}\right\} \subset \operatorname{Stab}_{\varepsilon}\left\{y, y^{\prime}\right\}
$$

A prova é uma consequência imediata da convexidade da distância. Prova.[Teorema 8.3]

O nosso objetivo é provar que $\operatorname{Stab}_{\varepsilon}\left\{h_{n_{*}}{ }^{-2} \cdot w_{n}, h_{n_{*}}^{2} \cdot w_{n}\right\} \subset \operatorname{Cr}\left(\mathbb{P}_{\bar{k}}^{2}\right)$ é finito, usando o mesmo $\varepsilon=\varepsilon_{n}$ da Proposição 8.3. Com isso, teremos que $h_{n}$ satisfaz a propriedade WPD. E de fato, pela Proposição 8.4, temos

$$
\operatorname{Stab}_{\varepsilon_{n}}\left\{h_{n_{*}}^{-2} \cdot w_{n}, h_{n_{*}}^{2} \cdot w_{n}\right\} \subset \operatorname{Stab}_{\varepsilon_{n}}\left\{h_{n_{*}}^{-1} \cdot w_{n}, h_{n *} \cdot w_{n}\right\} \subset \operatorname{Stab}_{\varepsilon_{n}}\left\{w_{n}\right\},
$$

e pela Proposição 8.3, seus elementos são automorfismos. Basta mostrar que existem finitos automorfismos nesse conjunto. 
Seja $f \in \operatorname{Stab}_{\varepsilon_{n}}\left\{h_{n_{*}}{ }^{-2} \cdot w_{n}, h_{n_{*}}^{2} \cdot w_{n}\right\}$, então $f$ é tal que

$$
(x: y: z) \mapsto(a x+k y+b z: l x+c y+d z: h x+m y+j z) .
$$

Como $f \in \operatorname{Stab}_{\varepsilon_{n}}\left\{h_{n_{*}}^{-1} \cdot w_{n}, h_{n *} \cdot w_{n}\right\}$, temos

$$
\mathrm{d}\left(f_{\star} h_{n_{*}}^{-1} \cdot w_{n}, h_{n_{*}}^{-1} \cdot w_{n}\right) \leq \varepsilon_{n},
$$

e como $h_{n *}$ é uma isometria,

$$
\mathrm{d}\left(h_{n *} f_{\star} h_{n_{*}}^{-1} \cdot w_{n}, w_{n}\right) \leq \varepsilon_{n} .
$$

Em particular, temos que $h_{n} f h_{n}^{-1} \in \operatorname{Stab}_{\varepsilon_{n}}\left\{w_{n}\right\}$ e portanto é um automorfismo. Seja $C \subset \mathbb{P}^{2}$ a curva que é contraída em (1:0:0) por $h_{n}^{-1}$. Como $h_{n} f h_{n}^{-1}$ é um automorfismo, então $h_{n} f h_{n}^{-1}(C)=h_{n} f(1: 0: 0)$ deve ser uma curva. Isso só é possível se $f(1: 0: 0)=(1: 0: 0)$, caso contrário $h_{n} f(1: 0: 0)$ seria um único ponto. Isso implica que $l=h=0$. Analogamente, temos

$$
\mathrm{d}\left(f_{\star} h_{n *} \cdot w_{n}, h_{n *} \cdot w_{n}\right) \leq \varepsilon_{n}
$$

$h_{n}^{-1} f h_{n}$ é um automorfismo e $f(0: 1: 0)=(0: 1: 0)$, logo $k=m=0$. Podemos supor que $j=1$ e temos uma forma afim de descrever $f$ como

$$
(x, y) \mapsto(a x+b, c x+d) .
$$

Desenvolvendo os automorfismos $h_{n} f h_{n}^{-1}$ e $h_{n}^{-1} f h_{n}$, temos

$$
h_{n} f h_{n}^{-1}=\left(c x+d, x^{n}\left(c^{n}-a\right)+n c^{n-1} d x^{n-1}+\ldots+n c d^{n-1} x+a y+d^{n}-b\right)
$$

e também

$$
h_{n}^{-1} f h_{n}=\left(y^{n}\left(a^{n}-c\right)+n a^{n-1} b y^{n-1}+\ldots+n a b^{n-1} y+a x+d^{n}-b, a y+b\right) .
$$

Como se tratam de automorfismos afins, os coeficientes de $x^{n}$ e $y^{n}$ devem ser nulos, já que $n \geq 2$. Portanto $c^{n}=a$ e $a^{n}=c$ implica que $a$ e $c$ são raízes $\left(n^{2}-1\right)$-ésimas da unidade, totalizando no máximo $\left(n^{2}-1\right)^{2}$ combinações para o par $(a, c)$.

Além disso, quando $n \geq 3$, também se anulam os coeficientes de $x^{n-1}$ e $y^{n-1}$, isto é, $n c^{n-1} d=n a^{n-1} b=0$. Como $\operatorname{char}(k) \nmid n$, e $a$ e $c$ são raízes da unidade, $b=d=0$. Nesse caso, temos no máximo $\left(n^{2}-1\right)^{2}$ tais automorfismos $f$. 
Quando $n=2$, vamos usar o fato de que $h_{n}^{2} f h_{n}^{-2}$ e $h_{n}^{-2} f h_{n}^{2}$ também devem ser automorfismos, que obtemos de forma análoga a $h_{n} f h_{n}^{-1}$ e $h_{n}^{-1} f h_{n}$, usando que $f \in \operatorname{Stab}_{\varepsilon_{n}}\left\{h_{n_{*}}^{-2} \cdot w_{n}, h_{n_{*}}^{2} \cdot w_{n}\right\}$. Novamente de maneira análoga, vamos precisar que os automorfismo $h_{n} f h_{n}^{-1}$ e $h_{n}^{-1} f h_{n}$ preservem os pontos $P_{0}$ e $P_{1}$ (do mesmo jeito que provamos que $f$ faria). Então pelo desenvolvimento de $h_{n} f h_{n}^{-1}$ e $h_{n}^{-1} f h_{n}$, teremos $2 c d=2 a b$ e novamente $b=d=0$. Temos portanto no máximo $\left(n^{2}-1\right)^{2}=9$ tais automorfismos $f$. 


\section{9}

\section{Referências bibliográficas}

BESTVINA, M.; FUJIWARA, K. Bounded cohomology of subgroups of mapping class groups. Geometry and Topology, Mathematical Sciences Publishers, v. 6, n. 1, p. 69-89, 2002.

CANTAT, S.; LAMY, S. Normal subgroups in the cremona group. Acta Mathematica, International Press of Boston, v. 210, n. 1, p. 31-94, 2013.

CASTELNUOVO, G. Le trasformazioni generatrici del gruppo cremoniano nel piano. [S.I.]: Turin R. Accad. d. Sci., 1901.

DAHMANI, F.; GUIRARDEL, V.; OSIN, D. Hyperbolically embedded subgroups and rotating families in groups acting on hyperbolic spaces. Mem. Amer. Math. Soc., American Mathematical Society, v. 245, n. 1156, p. v+152, 2017.

DILLER, J.; FAVRE, C. Dynamics of bimeromorphic maps of surfaces. American Journal of Mathematics - AMER J MATH, Johns Hopkins University Press, v. 123, n. 6, p. 1135-1169, 2001.

DOLGACHEV, I. V.; ISKOVSKIKH, V. A. Finite subgroups of the plane cremona group. In: Algebra, Arithmetic, and Geometry: Volume I: In Honor of Yu. I. Manin. Birkhäuser Boston, 2009. p. 443-548. ISBN 978-0-8176-4745-2. Disponível em: <https://doi.org/10.1007/978-0-8176-4745-2_11>.

HARTSHORNE, R. Algebraic Geometry. [S.I.]: Springer-Verlag New York, 1977.

KLEIMAN, S.; ALTMAN, A. A Term of Commutative Algebra. Worldwide Center of Mathematics, LLC, 2018. ISBN 9780988557215. Disponível em: < https: //books.google.com.br/books?id=KyBokQEACAAJ>.

KOLLáR, J.; SMITH, K. E.; CORTI, A. Rational and Nearly Rational Varieties. [S.I.]: Cambridge University Press, 2004.

LANG, S. Algebra. 3. ed. [S.I.]: Springer-Verlag New York, 2002. (Graduate Texts in Mathematics 211). ISBN 9780387953854,038795385X.

LONJOU, A. Non simplicité du groupe de cremona sur tout corps. Annales de I'Institut Fourier, Association des Annales de l'institut Fourier, v. 66, n. 5, p. 2021-2046, 2016.

PAUPERT, J. Introduction to Hyperbolic Geometry. 2016. < https://math. la.asu.edu/ paupert/HyperbolicGeometryNotes.pdf $>$.

SHAFAREVICH, I. R. Basic Algebraic Geometry 1. [S.I.]: Springer-Verlag Berlin Heidelberg, 1994. ISBN 978-3-642-37955-0. 